\title{
Unrectifiable 1-sets have vanishing analytic capacity
}

\section{Guy David}

Résumé. On complète la démonstration d'une conjecture de Vitushkin: si $E$ est une partie compacte du plan complexe de mesure de Hausdorff unidimensionelle nulle, alors $E$ est de capacité analytique nulle (toute fonction analytique bornée sur le complémentaire de $E$ est constante) si et seulement si $E$ est totalement non rectifiable (l'intersection de $E$ avec toute courbe de longueur finie est de mesure de Hausdorff nulle). Comme dans un papier précédent avec P. Mattila, la démonstration repose sur un critère de rectifiabilité utilisant la courbure de Menger, et une extension d'une construction de M. Christ. L'élément nouveau principal est une généralisation du théorème $T(b)$ sur certains espaces qui ne sont pas nécessairement de type homogène.

Abstract. We complete the proof of a conjecture of Vitushkin that says that if $E$ is a compact set in the complex plane with finite 1-dimensional Hausdorff measure, then $E$ has vanishing analytic capacity (i.e., all bounded analytic functions on the complement of $E$ are constant) if and only if $E$ is purely unrectifiable (i.e., the intersection of $E$ with any curve of finite length has zero 1-dimensional Hausdorff measure). As in a previous paper with P. Mattila, the proof relies on a rectifiability criterion using Menger curvature, and an extension of a construction of M. Christ. The main new part is a generalization of the $T(b)$-Theorem to some spaces that are not necessarily of homogeneous type. 


\section{Introduction.}

The main goal of this paper is to complete the proof of Vitushkin's conjecture on 1-sets of vanishing analytic capacity.

Let $E$ be a compact set in the complex plane. We say the $E$ has vanishing analytic capacity if all bounded analytic functions on $\mathbb{C} \backslash E$ are constant. Ahlfors ([Ah]) proved that $E$ has vanishing analytic capacity if and only if it is removable for bounded analytic functions, i.e., if for all choices of an open set $\Omega \subset E$ and a bounded analytic function $f$ on $\Omega \backslash E, f$ has an analytic extension to $\Omega$.

It was conjectured by Vitushkin ([Vi]) that if $E$ is a compact set such that $0<H^{1}(E)<+\infty$, then $E$ has vanishing analytic capacity if and only if $E$ is totally unrectifiable (or irregular in the terminology of Besicovitch), which means that $H^{1}(E \cap G)=0$ for all rectifiable curves $G$. Here $H^{1}$ denotes one-dimensional Hausdorff measure. Actually, Vitushkin's conjecture also said something about the case when $H^{1}(K)=+\infty$, but this part turned out to be false ([Ma1]).

The first half of this conjecture was obtained as a consequence of A. P. Calderón's result on the boundedness of the Cauchy integral operator on $L^{2}(\Gamma)$ when $\Gamma$ is a $C^{1}$-curve (or even a Lipschitz graph with small constant) in the plane ([Ca]). Indeed, if $E$ is a compact subset of a rectifiable curve and $H^{1}(E)>0$, there is a $C^{1}$-curve $\Gamma$ such that $H^{1}(E \cap F)>0$, and one can use Calderón's theorem and a nice duality argument of $\mathrm{Uy}([\mathrm{Uy}])$ or Havin and Havinson ([HH]) to find non constant bounded analytic functions on $\mathbb{C} \backslash(E \cap F)$. Thus $E$ cannot be removable for bounded analytic functions if $H^{1}(E \cap G)>0$ for some rectifiable curve $G$. See for instance [Ch1] for a recent treatment of this result.

Our main result is as follows.

Theorem 1.1. Let $E \subset \mathbb{C}$ be a compact set such that $H^{1}(E)<+\infty$ and $E$ is totally unrectifiable. Then $E$ has vanishing analytic capacity.

Progress in the direction of Theorem 1.1 has been quite slow for some time, because one was not able to relate nicely information on the Cauchy kernel (typically, the existence of a bounded function on $E$ whose Cauchy integral is bounded on $\mathbb{C} \backslash E$ ) to the geometry of $E$. Then M. Melnikov introduced "Menger curvature" in connection to analytic capacity $([\mathrm{Me}])$. This was rapidly followed by a result on the Cauchy operator $([\mathrm{MV}])$ and the proof of Theorem 1.1 in the special case when 
$E$ is Ahlfors-regular ([MMV]). This last means that there is a constant $C>0$ such that

$$
C^{-1} r \leq H^{1}(E \cap B(x, r)) \leq C r,
$$

for all $x \in E$ and $0<r \leq \operatorname{diam} E$.

H. Pajot ([Pa]) observed that Ahlfors-regularity can be replaced with the weaker condition that

$$
\left\{\begin{array}{l}
\liminf _{r \rightarrow 0}\left(r^{-1} H^{1}(E \cap B(x, r))\right)>0, \\
\limsup _{r \rightarrow 0}\left(r^{-1} H^{1}(E \cap B(x, r))\right)<+\infty, \quad \text { for all } x \in E .
\end{array}\right.
$$

(This last is a sufficient condition for $E$ to be contained in a countable union of Ahlfors-regular sets.) The method for these papers uses the miraculous positivity properties of Menger curvature, but also relies on standard Calderón-Zygmund techniques such as the $T(1)$-theorem. For these it is very useful to know that $E$ is Ahlfors-regular, or at least that the restriction of $H^{1}$ to $E$ is doubling, i.e., that $H^{1}(E \cap B(x, 2 r)) \leq$ $C H^{1}(E \cap B(x, r))$ for all $x \in E$ and $0<r \leq \operatorname{diam} E([\mathrm{Li}])$.

It turns out that the general Calderón-Zygmund techniques used by [Ch2] and [MMV] do not fail in the general case when $0<H^{1}(E)<$ $+\infty$, but merely become much more painful to apply. This was (partially) observed in [DM], where the analogue of Theorem 1.1 for Lipschitz harmonic functions (instead of bounded analytic) is proved. The present paper will rely on the construction of [DM].

Before we start a short description of the argument, let us observe that it is very easy to show that $E$ is removable for bounded analytic functions if $H^{1}(E)=0$ (apply Cauchy's formula on curves of arbitrarily small lengths that surround $E$ ). Also, compact sets of dimension $d>1$ are not removable: one can construct bounded analytic functions by taking Cauchy integrals of positive measures $\mu$ such that $\mu(B(x, r)) \leq C r^{d^{\prime}}$ for some $d^{\prime} \in(1, d)$; such measures can be obtained from Frostman's lemma. Thus the only unclear situation left is when $E$ has dimension 1 and $H^{1}(E)=+\infty$. See for instance [Ga], [Ch1], [Ma2], or [Vi] for general information about analytic capacity.

Let us now describe our strategy for proving Theorem 1.1. More details will be given in the course of the paper, but the reader may want to use this description to avoid getting lost in unimportant complications.

Let $E \subset \mathbb{C}$ be compact, and assume that $H^{1}(E)<+\infty$ and $E$ does not have vanishing analytic capacity; we want to prove that $E$ has 
a non trivial rectifiable piece. By easy manipulations, we can find a bounded analytic function $h$ on $\mathbb{C} \backslash E$ such that $h(\infty)=0$ and $h^{\prime}(\infty)=$ $\lim _{z \rightarrow \infty} z h(z)=: a>0$. It is not hard to show that

$$
h(z)=\int_{E} \frac{f(y) d \mu(y)}{z-y}, \quad \text { for } z \in \mathbb{C} \backslash E,
$$

where $\mu$ denotes the restriction of $H^{1}$ to $E$ (i.e., $\mu(A)=H^{1}(A \cap E)$ for all Borel sets $A$ ) and $f$ is some bounded measurable function on $E$. This is Theorem 19.9 in [Ma2]. To prove it one surrounds $E$ by a sequence of (finitely connected) curves $\Gamma_{n}$ and one applies Cauchy's formula to them; eventually $f d \mu$ comes out as a weak limit of measures $h(y) d y$ on curves $\Gamma_{n}$.

The first stage of our argument consists in replacing $f d \mu$ with a new finite measure $g d \nu$ with the following properties:

$$
0 \leq \nu(B(x, r)) \leq C r, \quad \text { for all } x \in \mathbb{C} \text { and } r>0
$$

$\mathrm{g}$ is bounded acccretive, i.e.,

$$
\begin{gathered}
|g(x)| \leq C, \quad \operatorname{Re} g(x) \geq C^{-1} \text { for all } x \in \mathbb{C}, \\
\int g d \nu=\int f d \mu=a>0
\end{gathered}
$$

there is a Borel set $F \subset E$ such that

$$
C^{-1} \mu \leq \nu \leq \mu \text { on } F \text { and } \nu(F) \geq \frac{a}{2}
$$

(the first half means that $C^{-1} \mu(A) \leq \nu(A) \leq \mu(A)$ for all Borel subsets $A$ of $F$ ), and

the Cauchy integral of $g d \nu$ lies

in an appropriate space $\mathrm{BMO}(d \nu)$.

The measure $g d \nu$ will be imported directly from $[\mathrm{DM}]$, where it was constructed for very similar reasons (see in particular Theorem 2.4 in $[\mathrm{DM}])$; the properties (1.5)-(1.8) are the same as (2.5)-(2.8) in [DM], and (1.9) will have to be made more precise and proved, starting from the corresponding $L^{2}$-estimate (2.9) in [DM]. The construction of $g d \nu$ is very similar in spirit to a construction of M. Christ ([Ch2]), who used 
it to show that if $E$ is a regular set with positive analytic capacity, then there is another Ahlfors-regular set $G$ such that $H^{1}(E \cap G)>0$ and for which the Cauchy integral defines a bounded operator on $L^{2}(G)$. At that time, $[\mathrm{MMV}]$ did not exist, and so M. Christ could not conclude that $G$ is uniformly rectifiable. The proof of boundedness of the Cauchy operator on $L^{2}(G)$ was directly deduced from the analogues of (1.6) and (1.9) by the $T(b)$-theorem (on $G$ ).

The construction of $g d \nu$ in [Ch2] and [DM] relies on the existence on $E$ of an analogue of the decomposition of $\mathbb{R}^{n}$ into dyadic cubes. The general scheme is to replace $f d \mu$ by measures that live on small circles on (maximal) "cubes" $Q \subset E$ where $\operatorname{Re} \int f d \mu$ is a little too small. The construction is less pleasant in $[\mathrm{DM}]$ than in [Ch2], because one has to find slightly different ways to deal with the "small boundary property" of the constructed "dyadic cubes" when $\mu$ is not doubling. Nonetheless the spirit is the same.

In [DM] we could not continue as in [Ch2], because we did not have an appropriate $T(b)$-theorem. This is the reason why we restricted to Lipschitz harmonic capacity. If $H^{1}(E)<+\infty$ and $E$ has positive Lipschitz harmonic capacity, then we can get $f d \mu$ (and then $g d \nu$ ) as above, but with $f$ real-valued (and hence $g(x) \geq C^{-1}$ ) in addition. Then we do not need Stage 2 below, and we can use the argument of Stage 3 below to find that $F$ is rectifiable (and hence that $E$ is not totally unrectifiable).

In the present situation, $g$ is not necessarily positive and we cannot apply directly the positivity argument with Menger curvature from [MMV] (see below), as in [DM]. So we'll prove a $T(b)$-theorem on $\tilde{E}=\operatorname{supp}(\nu)$ and apply it to the truncated operators $T_{\varepsilon}$ given by

$$
T_{\varepsilon} f(x)=\int_{|x-y|>\varepsilon} \frac{f(y) d \nu(y)}{x-y},
$$

to get uniform bounds on the norm of $T_{\varepsilon}$ on $L^{2}(\tilde{E}, d \nu)$. Once again, the proof of the $T(b)$-theorem of Section 3 will follow rather classical outlines: we shall use the dyadic cubes from [DM], construct a version of the Haar system adapted to those cubes and the accretive function $b=$ $g$, remove a "paraproduct" that takes care of $T b$ and $T^{t} b$, and prove that the matrix of the remaining operator in the modified Haar system has sufficient decay away from the diagonal to allow a use of Schur's lemma. This is the same program as in the proof of the (standard) $T(b)$-theorem by Coifman-Semmes ([CJS]) or Auscher-Tchamitchian ([AT]). See also $[\mathrm{Da}]$ or $[\mathrm{My}]$ for a presentation of this scheme and [DJS] for the original 
$T(b)$ paper. Here again, the fact that $\mu$ is not necessarily doubling will create trouble, but altogether nothing dramatic. See sections 2-9 for the details.

We shall also need to spend some time checking that our $T(b)$ theorem applies to the space $(\tilde{E}, d \nu)$ and the function $b=g$ (see sections 10-13). In particular we'll have to build cubes adapted to $d \nu$, and then check the appropriate version of (1.9).

At the end of this (call it Stage 2), we know that the truncated Cauchy operators $T_{\varepsilon}$ are bounded on $L^{2}(d \nu)$, with bounds that do not depend on $\varepsilon$. In particular,

$$
\left\|T_{\varepsilon} 1\right\|_{L^{2}(d \nu)}^{2} \leq C,
$$

where $C$ does not depend on $\varepsilon>0$. A brutal expansion of (1.11) gives that

$$
\int_{x \in \tilde{E}}\left(\int_{|x-y|>\varepsilon} \frac{d \nu(y)}{x-y}\right)\left(\int_{|x-z|>\varepsilon} \frac{d \nu(z)}{\overline{x-z}}\right) d \nu(x) \leq C .
$$

(There is no problem of convergence here and in the lines that follows, because $\nu$ is a finite measure.) The domain of integration in (1.12) is $U(\varepsilon) \cup V(\varepsilon)$, where

$$
U(\varepsilon)=\left\{(x, y, z) \in \tilde{E}^{3}: \varepsilon<|x-y|,|x-z|,|y-z|\right\}
$$

and

(1.14) $V(\varepsilon)=\left\{(x, y, z) \in \tilde{E}^{3}:|x-y|>\varepsilon,|x-z|>\varepsilon\right.$ and $\left.|y-z| \leq \varepsilon\right\}$.

A fairly brutal computation gives that

$$
\iiint_{V(\varepsilon)} \frac{d \nu(x) d \nu(y) d \nu(z)}{|x-y||x-z|} \leq C,
$$

see $[\mathrm{MV},(5)]$, and note that the (very short) proof only uses (1.5). Thus

$$
\left|\iiint_{U(\varepsilon)} \frac{d \nu(x) d \nu(y) d \nu(z)}{(x-y)(\overline{x-z})}\right| \leq C .
$$

Now we want to use the following nice formula [Me]: for each triple $\left(z_{1}, z_{2}, z_{3}\right)$ of distinct points of $\mathbb{C}$,

$$
\sum_{\sigma \in G_{3}} \frac{1}{\left(z_{\sigma(1)}-z_{\sigma(2)}\right)\left(\overline{z_{\sigma(1)}-z_{\sigma(3)}}\right)}=c^{2}\left(z_{1}, z_{2}, z_{3}\right),
$$


where we sum over the group $G_{3}$ of permutations of $\{1,2,3\}$ and $c\left(z_{1}, z_{2}, z_{3}\right)$ denotes the Menger curvature of the triple $\left(z_{1}, z_{2}, z_{3}\right)$, i.e., the inverse of the radius of the circle that goes through $z_{1}, z_{2}, z_{3}$. (When the three points are on a line, set $c\left(z_{1}, z_{2}, z_{3}\right)=0$.) This is $[\mathrm{Me},(19)$, p. 842]. Because the integral in (1.16) is invariant under permutations of $x, y, z$, we can use (1.17) to get that

$$
\iiint_{U(\varepsilon)} c^{2}(x, y, z) d \nu(x) d \nu(y) d \nu(z) \leq C,
$$

still with a constant $C$ that does not depend on $\varepsilon$. Hence (by positivity),

$$
c^{2}(\nu)=: \iiint_{\tilde{E}^{3}} c^{2}(x, y, z) d \nu(x) d \nu(y) d \nu(z) \leq C .
$$

We shall call $c(\nu)$ the Melnikov curvature of the measure $\nu$.

At this point we can use a theorem of David and Léger ([Lé]), which says that if $\nu$ is a finite measure on $\mathbb{C}$ such that (1.5) holds, $c^{2}(\nu)<+\infty$, and if $\tilde{E}$, the support of $\nu$, has finite $H^{1}$-measure, then $\nu$ is rectifiable. This means that $\tilde{E}$ is contained in a countable union of rectifiable curves, plus possibly a set of $\nu$-measure zero. The set $\tilde{E} \cap F$, where $F$ is as in (1.8), is also rectifiable, and hence meets some rectifiable curve on a set of $H^{1}$-measure greater than 0 . This third stage completes the (sketch of) proof of Theorem 1.1.

Theorem 1.1 leaves open the characterization of vanishing analytic capacity for compact subsets of the plane such that $H^{1}(E)=+\infty$ but dimension $(E)=1$. The obvious generalization of Vitushkin's conjecture where one would demand that

$$
H^{1}\left(\pi_{\theta}(E)\right)=0, \quad \text { for almost every } \theta \in \mathbb{R}
$$

where $\pi_{\theta}$ denotes the orthogonal projection onto the line of direction $e^{i \theta}$, does not work. P. Mattila ([Ma1]) showed that (1.20) is not preserved when we replace $E$ with its image under conformal mappings, while vanishing analytic capacity is. P. Jones and T. Murai ([JM]) later found examples of compact sets $E \subset \mathbb{C}$ with positive analytic capacity and such that (1.20) holds. It is not known yet whether there are compact sets of vanishing analytic capacity for which (1.20) does not hold. M. Melnikov likes to conjecture that compact sets $E$ have positive analytic capacity if and only if there is a (nonzero) positive measure $\nu$ supported on $E$ and such that $\nu(B(x, r)) \leq C r$ for all $x \in E$ and $r>0$, 
and $c^{2}(\nu)<+\infty$. Note that the "if" part of this conjecture is proved in $[\mathrm{Me}]$.

\section{Construction of a Haar system.}

In this section we are given a Borel subset $E$ of some $\mathbb{R}^{N}$ and a finite Borel measure $\mu$ on $E$. We are also given a sequence of partitions of $E$ into Borel subsets $Q, Q \in \Delta_{k}, k \geq 0$, with the following properties: $Q \in \Delta_{k}$,

2.1) for each integer $k \geq 0, E$ is the disjoint union of the sets $Q$,

2.2) if $0 \leq k<\ell, Q \in \Delta_{k}$, and $R \in \Delta_{\ell}$, then either $Q \cap R=\varnothing$ or else $R \subset Q$,

2.3) $\mu(Q)>0$ for all $Q \in \Delta_{k}$ and all $k \geq 0$,

2.4) $\operatorname{diam} Q \leq C_{0} A^{-k}$ for all $k \geq 0$ and $Q \in \Delta_{k}$,

2.5) for each $k \geq 0$ and each $Q \in \Delta_{k}$, the number of $R \in \Delta_{k+1}$ such that $R \subset Q$ is $\leq C_{0}$.

Here $C_{0}$ and $A>1$ are two constants that do not depend on $k$ or $Q$, and $\operatorname{diam} Q$ is the diameter of $Q$. The sets $Q, Q \in \bigcup_{k} \Delta_{k}$, will be called cubes, or dyadic cubes (even though they should probably be called $A$-adic.) In the later sections, more will be required from these cubes, but the properties 2.1)-2.5) will be enough for the moment.

For each cube $Q$, we shall denote by $k(Q)$ the integer $k$ such that $Q \in \Delta_{k}$, and by $d(Q)=A^{-k(Q)}$ its official approximate size. We should mention now that $\operatorname{diam} Q$ may be much smaller than $d(Q)$, and also that a given subset of $E$ could be equal to $Q$ for a few different cubes $Q$ coming from different generations $k(Q)$. When we talk about a cube $Q$, we shall always mean both the set $Q$ itself and the knowledge of the generation $k(Q)$.

If $Q$ is a cube of generation $k(Q) \geq 1$, then there is a unique cube $\hat{Q} \in \Delta_{k(Q)-1}$ which contains $Q$, and which we'll call the parent of $Q$. The children of $Q$ are the cubes $R \in \Delta_{k(Q)+1}$ that are contained in $Q$. We shall denote by $F(Q)$ the set of children of $Q$. Note that in some instances $F(Q)$ will be reduced to only one child, the set $Q$ itself. At any rate, 2.5$)$ says that $F(Q)$ never has more than $C_{0}$ elements.

In this section we want to construct a Riesz basis of $L^{2}(E, d \mu)$ which is adapted to the above decomposition of $E$ into cubes, and a given accretive function $b$. This Riesz basis will be analogous to the 
Haar basis, which corresponds to the case of $E=[0,1] \subset \mathbb{R}$, equipped with the Lebesgue measure, the usual dyadic intervals, and $b \equiv 1$. The construction given below is very similar to one initially used for [CJS] or [AT], but we shall need to repeat the argument to convince the reader that nothing more than 2.1)-2.5) is needed. For personal convenience reasons, we shall stay pretty close to the argument given in [Da].

Our function $b$ is Borel-measurable, complex-valued, and bounded and accretive. This means that

$$
|b(x)| \leq C \quad \text { and } \quad \operatorname{Re} b(x) \geq C^{-1}, \quad \text { for all } x \in E .
$$

In fact, we shall only use the paraaccretivity condition that $b$ is bounded and

$$
\left|\int_{Q} b d \mu\right| \geq C^{-1} \mu(Q), \quad \text { for all cubes } Q
$$

but this will not matter for our only application.

We start our construction with the definition of a few projection operators. For $x \in E$ and $k \geq 0$, denote by $Q_{k}(x)$ the cube of $\Delta_{k}$ that contains $x$. Then set, for each $f \in L^{2}(E, d \mu)$,

$$
E_{k} f(x)=\mu\left(Q_{k}(x)\right)^{-1} \int_{Q_{k}(x)} f d \mu .
$$

This is the standard orthogonal projection on the set of functions that are constant on each cube $Q \in \Delta_{k}$. Also set

$$
D_{k}=E_{k+1}-E_{k}, \quad k \geq 0,
$$

and then define the corresponding twisted operators $F_{k}$ and $Z_{k}$ by

$$
F_{k} f(x)=\left(\int_{Q_{k}(x)} b d \mu\right)^{-1} \int_{Q_{k}(x)} f b d \mu
$$

and

$$
Z_{k}=F_{k+1}-F_{k}
$$

We need a few easy facts concerning these operators. First,

$$
\int_{Q}\left(F_{k} f\right) b d \mu=\int_{Q} f b d \mu, \quad \text { for all } Q \in \Delta_{k},
$$


which is clear from (2.10). Next,

$$
F_{j} F_{k}=F_{j \wedge k},
$$

with $j \wedge k=\min \{j, k\}$. When $j \geq k$, we observe that $F_{k} f$ is constant on all cubes $Q \in \Delta_{k}$, and hence also on cubes of $\Delta_{j}$. Then $F_{j} F_{k} f=F_{k} f$. When $j<k,(2.12)$ says that

$$
\int_{Q}\left(F_{k} f\right) b d \mu=\int_{Q} f b d \mu,
$$

for all $Q \in \Delta_{k}$, and hence all cubes $Q \in \Delta_{j}$. Then $F_{j} F_{k} f=F_{j} f$, by definition of $F_{j}$. This proves (2.13). Next

$$
Z_{j} Z_{k}=\delta_{j, k} Z_{j}
$$

because

$Z_{j} Z_{k}=\left(F_{j+1}-F_{j}\right)\left(F_{k+1}-F_{k}\right)=F_{j+1} F_{k+1}-F_{j} F_{k+1}-F_{j+1} F_{k}+F_{j} F_{k}$

A brutal computation using (2.13) gives the result.

Let us also check that

$$
\int\left(Z_{k} u\right)\left(Z_{\ell} v\right) b d \mu=0, \quad \text { for } u, v \in L^{2}(d \mu) \text { and } k \neq \ell .
$$

We can assume that $k>\ell$. Since $Z_{\ell} v$ is constant on each cube of $\Delta_{k}$, it is enough to show that

$$
\int_{Q}\left(Z_{k} u\right) b d \mu=0, \quad \text { for all } Q \in \Delta_{k}
$$

This last holds because

$$
\int_{Q}\left(F_{k} u\right) b d \mu=\int_{Q}\left(F_{k+1} u\right) b d \mu=\int_{Q} f b d \mu
$$

by $(2.12)$.

Next we check that $E_{0}$ and the $D_{k}, k \geq 0$, provide an orthonormal decomposition of $L^{2}(d \mu)$. First observe that if $\mathcal{E}$ denotes the set of (finite) linear combinations of characteristic functions of cubes, then

$$
\mathcal{E} \text { is dense in } L^{2}(d \mu) \text {. }
$$


This is an easy consequence of (2.4), or more precisely of the fact that we can decompose $E$ into disjoint unions of cubes of arbitrarily small diameters, because continuous functions are dense in $L^{2}(d \mu)$. Then

$$
f=\lim _{k \rightarrow \infty} E_{k} f \quad \text { (with convergence in } L^{2}(d \mu) \text { ), }
$$

for all $f \in L^{2}(d \mu)$, because this is obviously true when $f \in \mathcal{E}$, and the operators $E_{k}$ are uniformly bounded. Also, the decomposition

$$
E_{k} f=E_{0} f+\sum_{\ell=0}^{k-1} D_{\ell} f
$$

is orthogonal. The orthogonality of the $D_{\ell}$ 's among themselves comes for instance from (2.15) with $b \equiv 1$, and they are orthogonal to $E_{0}$ by (2.16) with $b \equiv 1$. Because of this and (2.18),

$$
\|f\|_{2}^{2}=\left\|E_{0} f\right\|_{2}^{2}+\sum_{\ell \geq 0}\left\|D_{\ell} f\right\|_{2}^{2}
$$

for all $f \in L^{2}(d \mu)$.

We want to prove similar estimates for $F_{0}$ and the $Z_{\ell}$ 's, but first we need a few facts about Carleson measures.

Definition 2.21. A Carleson measure on $E \times \mathbb{N}$ is a measure $\nu=$ $\left\{\nu_{k}\right\}_{k \geq 0}$ on $E \times \mathbb{N}$ such that

$$
\nu(Q \times\{k \in \mathbb{N}: k \geq k(Q)\})=: \sum_{k \geq k(Q)} \nu_{k}(Q) \leq C \mu(Q),
$$

for all cubes $Q$, and with a constant $C$ that does not depend on $Q$.

Recall that $k(Q)$ denotes the generation of $Q$. The definition is very analogous to the definition of discrete Carleson measures on the upper half space; one should not be disturbed by the fact that the role of $t>0$ is played by $A^{-k}, k \in \mathbb{N}$, in our situation. Here is Carleson's theorem in our context.

Lemma 2.23. Let $\nu=\left\{\nu_{k}\right\}_{k \geq 0}$ be a Carleson measure on $E \times \mathbb{N}$. Also let $f \in L^{2}(d \mu)$ and a sequence $\left\{f_{k}\right\}_{k \in \mathbb{N}}$ of functions be given. If

$$
\left|f_{k}(x)\right| \leq \mu\left(Q_{k}(x)\right)^{-1} \int_{Q_{k}(x)}|f| d \mu
$$


for all $k \geq 0$ and $x \in E$, then

$$
\int\left(\left|f_{k}\right|^{2}\right)_{k} d \nu=: \sum_{k} \int\left|f_{k}\right|^{2} d \nu_{k} \leq C\|f\|_{2}^{2}
$$

To prove this, we first need estimates on the maximal function

$$
f^{*}(x)=\sup _{k} \mu\left(Q_{k}(x)\right)^{-1} \int_{Q_{k}(x)}|f| d \mu .
$$

We start with the usual weak- $L^{1}$ estimate. Let $f \in L^{1}(d \mu)$ and $\lambda>0$ be given, and set $\mathcal{O}(\lambda)=\left\{x \in E: f^{*}(x)>\lambda\right\}$. Also denote by $\mathcal{M}_{\lambda}$ the collection of maximal cubes $Q$ with the property that

$$
\int_{Q}|f| d \mu>\lambda \mu(Q)
$$

(These are the cubes such that (2.27) holds and either $Q \in \Delta_{0}$ or else none of the ancestors of $Q$ satisfies (2.27).) By definitions, the cubes $Q$ are disjoint (because they are maximal) and cover exactly $\mathcal{O}(\lambda)$. Then

$$
\mu(\mathcal{O}(\lambda))=\sum_{Q \in \mathcal{M}_{\lambda}} \mu(Q) \leq \lambda^{-1} \sum_{Q \in \mathcal{M}_{\lambda}} \int_{Q}|f| d \mu \leq \lambda^{-1}\|f\|_{1} .
$$

Thus the maximal operator $f \longrightarrow f^{*}$ maps $L^{1}(d \mu)$ boundedly into weak- $L^{1}(d \mu)$. Since it is also clearly bounded on $L^{\infty}(d \mu)$, real interpolation gives that

$$
\left\|f^{*}\right\|_{2} \leq C\|f\|_{2}, \quad \text { for } f \in L^{2}(d \mu) .
$$

Now let $f$ and $\left\{f_{k}\right\}$ be as in the lemma, and set

$$
\mathcal{U}(\lambda)=\left\{(x, k) \in E \times \mathbb{N}:\left|f_{k}(x)\right|>\lambda\right\},
$$

for each $\lambda>0$. If $(x, k) \in \mathcal{U}(\lambda)$, then

$$
\mu\left(Q_{k}(x)\right)^{-1} \int_{Q_{k}(x)}|f| d \mu \geq\left|f_{k}(x)\right|>\lambda
$$

by (2.24), and hence $Q_{k}(x)$ is contained in one of the cubes of $\mathcal{M}_{\lambda}$. Thus

$$
U(\lambda) \subset \bigcup_{Q \in \mathcal{M}_{\lambda}} Q \times\{k \geq k(Q)\}
$$


and then

$$
\begin{aligned}
\nu(\mathcal{U}(\lambda)) & \leq \sum_{Q \in \mathcal{M}_{\lambda}} \nu(Q \times\{k \geq k(Q)\}) \\
& \leq C \sum_{Q \in \mathcal{M}_{\lambda}} \mu(Q)=C \mu(\mathcal{O}(\lambda)),
\end{aligned}
$$

where $\mathcal{O}(\lambda)$ is as above, and by (2.22) and the first part of (2.28).

Thus the function of repartition of $\left\{f_{k}\right\}_{k>0}$ for the measure $\nu$ is dominated by the function of repartition of $f^{*}$ for $\mu$; the desired estimate (2.25) follows from this and the maximal theorem (2.29). This proves Lemma 2.23.

Lemma 2.33. For every $f \in L^{2}(d \mu)$,

$$
f=F_{0} f+\sum_{k \geq 0} Z_{k} f
$$

where the series converges in $L^{2}(d \mu)$, and

$$
C^{-1}\|f\|_{2}^{2} \leq\left\|F_{0} f\right\|_{2}^{2}+\sum_{k \geq 0} \int\left|Z_{k} f\right|^{2} d \mu \leq C\|f\|_{2}^{2}
$$

Of course the constant $C$ is not allowed to depend on $f$; it depends only on the accretivity constant in (2.6).

The formula (2.34) obviously holds when $f \in \mathcal{E}$ (and then the sum is finite), because $F_{k} f=f$ as soon as $f$ is constant on all the cubes of $\Delta_{k}$. The general case follows by density of $\mathcal{E}$, plus the fact that the operators $F_{k}$ are uniformly bounded on $L^{2}$, by their definition (2.10) and the accretivity condition (2.6). (Look at the effect of $F_{k}$ on each cube $Q \in \Delta_{k}$ separately.)

Now we want to prove the second inequality in (2.35). Write

$$
\begin{aligned}
Z_{k} f= & F_{k+1} f-F_{k} f \\
= & \left(E_{k+1} b\right)^{-1} E_{k+1}(b f)-\left(E_{k} b\right)^{-1} E_{k}(b f) \\
= & \left(\left(E_{k+1} b\right)^{-1}-\left(E_{k} b\right)^{-1}\right) E_{k+1}(b f) \\
& +\left(E_{k} b\right)^{-1}\left(E_{k+1}(b f)-E_{k}(b f)\right)
\end{aligned}
$$


and then use the fact that $\left(E_{k+1} b\right)^{-1}\left(E_{k} b\right)^{-1}$ is bounded because of (2.7) to get that

$$
\left|Z_{k} f\right|^{2} \leq C\left|D_{k} b\right|^{2}\left|E_{k+1}(b f)\right|^{2}+C\left|D_{k}(b f)\right|^{2} .
$$

We can easily take care of the second piece, because

$$
\sum_{k \geq 0} \int\left|D_{k}(b f)\right|^{2} d \mu=\sum_{k}\left\|D_{k} b f\right\|_{2}^{2} \leq\|b f\|_{2}^{2} \leq C\|f\|_{2}^{2},
$$

by (2.20). For the first piece, we want to use Lemma 2.23 with the sequence $\left\{f_{k}\right\}$ given by $f_{k}=E_{k}(b f), k \geq 1$. Obviously

$$
\left|E_{k}(b f)(x)\right| \leq \mu\left(Q_{k}(x)\right)^{-1} \int_{Q_{k}(x)}|b f| d \mu,
$$

for all $x \in E$, and so (2.24) holds (modulo an inessential constant).

We also want to take $\nu_{k}=\left|D_{k-1} b\right|^{2} d \mu$ for $k \geq 1$, and we have to check that this is a Carleson measure. Thus we take a cube $Q$ and try to estimate

$$
\sum_{k \geq k(Q)} \int_{Q}\left|D_{k-1} b\right|^{2} d \mu
$$

When $k>k(Q), D_{k-1} b=D_{k-1}\left(b \mathbf{1}_{Q}\right)$ on $Q$ by definitions, and so

$$
\begin{aligned}
\sum_{k>k(Q)} \int_{Q}\left|D_{k-1} b\right|^{2} d \mu & \leq \sum_{k} \int\left|D_{k-1}\left(b \mathbf{1}_{Q}\right)\right|^{2} d \mu \\
& \leq\left\|b \mathbf{1}_{Q}\right\|_{2}^{2} \\
& \leq C \mu(Q)
\end{aligned}
$$

by (2.20) and the fact that $b$ is bounded. The last term $\int_{Q}\left|D_{k(Q)} b\right|^{2} d \mu$ is at most $C \mu(Q)$ because $\left\|D_{k(Q)} b\right\|_{\infty} \leq 2\|b\|_{\infty}$, and so $\left\{\nu_{k}\right\}_{k \geq 1}$ defines a Carleson measure. By Lemma 2.23,

$$
\sum_{k \geq 1} \int\left|D_{k-1} b\right|^{2}\left|E_{k}(b f)\right|^{2} d \mu \leq C\|f\|_{2}^{2} .
$$

We are left with a last term, $k=0$. For this one,

$$
\int\left|D_{0} b\right|^{2}\left|E_{1}(b f)\right|^{2} d \mu \leq C\left\|E_{1}(b f)\right\|_{2}^{2} \leq C\|f\|_{2}^{2},
$$


by a brutal estimate. From (2.37), (2.38), (2.40) and (2.41) we deduce that

$$
\sum_{k \geq 0} \int\left|Z_{k} f\right|^{2} d \mu \leq C\|f\|_{2}^{2}
$$

Since we also have that

$$
\begin{aligned}
\left\|F_{0} f\right\|_{2}^{2} & =\sum_{Q \in \Delta_{0}}\left|\left(\int_{Q} b d \mu\right)^{-1}\left(\int_{Q} f b d \mu\right)\right|^{2} \mu(Q) \\
& \leq C \sum_{Q \in \Delta_{0}} \int_{Q}|f b|^{2} d \mu \\
& \leq C\|f\|_{2}^{2}
\end{aligned}
$$

by Cauchy-Schwarz, we get the second half of (2.35).

The first half of (2.35) will now follow by duality. We write

$$
f=F_{0} f+\sum_{k} Z_{k} f
$$

and

$$
b^{-1} \bar{f}=F_{0}\left(b^{-1} \bar{f}\right)+\sum_{k} Z_{k}\left(b^{-1} \bar{f}\right)
$$

as in (2.34), and then

$$
\|f\|_{2}^{2}=\int f\left(b^{-1} \bar{f}\right) b d \mu
$$

which we expand as suggested above. Note that for $k \neq \ell$,

$$
\int\left(Z_{k} f\right)\left(Z_{\ell}\left(b^{-1} \bar{f}\right)\right) b d \mu=0
$$

by (2.15), and also that

$$
\int\left(F_{0} f\right) Z_{k}\left(b^{-1} \bar{f}\right) b d \mu=\int F_{0}\left(b^{-1} \bar{f}\right) Z_{k}(f) b d \mu=0
$$


for all $k$ because $F_{0}(f)$ and $F_{0}\left(b^{-1} \bar{f}\right)$ are constant on cubes of $\Delta_{0}$ and by (2.16). Thus

$$
\begin{aligned}
\|f\|_{2}^{2} \leq & \left|\int\left(F_{0} f\right)\left(F_{0}\left(b^{-1} \bar{f}\right)\right) b d \mu\right|+\sum_{k}\left|\int\left(Z_{k} f\right)\left(Z_{k}\left(b^{-1} \bar{f}\right)\right) b d \mu\right| \\
\leq & C\left\|F_{0} f\right\|_{2}\left\|F_{0}\left(b^{-1} \bar{f}\right)\right\|_{2}+C \sum_{k}\left\|Z_{k} f\right\|_{2}\left\|Z_{k}\left(b^{-1} \bar{f}\right)\right\|_{2} \\
\leq & C\left(\left\|F_{0} f\right\|_{2}^{2}+\sum_{k}\left\|Z_{k} f\right\|_{2}^{2}\right)^{1 / 2} \\
& \cdot\left(\left\|F_{0}\left(b^{-1} \bar{f}\right)\right\|_{2}^{2}+\sum_{k}\left\|Z_{k}\left(b^{-1} \bar{f}\right)\right\|_{2}^{2}\right)^{1 / 2} \\
\leq & C\left(\left\|F_{0} f\right\|_{2}^{2}+\sum_{k}\left\|Z_{k} f\right\|_{2}^{2}\right)^{1 / 2}\left\|b^{-1} \bar{f}\right\|_{2}
\end{aligned}
$$

by Cauchy-Schwarz (twice) and the second half of (2.35) (applied to $\left.b^{-1} \bar{f}\right)$. Of course $\left\|b^{-1} \bar{f}\right\|_{2} \leq C\|f\|_{2}$, so we may divide both sides of (2.44) by $\|f\|_{2}$ (if $f \neq 0$ ) and get the first half of (2.35).

This completes the proof of Lemma 2.33.

For each cube $Q$, denote by $W^{+}(Q)$ the vector space of all functions $f$ that are supported on $Q$ and constant on each of the children of $Q$. Also let $W(Q)$ be the set of functions $f \in W^{+}(Q)$ such that

$$
\int_{Q} f b d \mu=0 \text {. }
$$

Let $r$ denote the number of children of $Q$; thus $1 \leq r \leq C_{0}$ by (2.5). The dimension of $W^{+}(Q)$ is obviously $r$. Since the condition (2.45) is not degenerate on $W^{+}(Q)$ (because $\mathbf{1}_{Q}$ does not satisfy $(2.45)$ ), $W(Q)$ is an $(r-1)$-dimensional space.

We want to find an appropriate basis of $W(Q)$. If $r=1$, i.e., if $Q$ has only one child, then $W(Q)=\{0\}$ and there is nothing to do. Otherwise we set $D=D(Q)=\{1,2, \ldots, r-1\}$ and look for a basis $\left\{h_{Q}^{\varepsilon}\right\}_{\varepsilon \in D}$ of $W(Q)$ such that

$$
\int_{Q} h_{Q}^{\varepsilon} h_{Q}^{\varepsilon^{\prime}} b d \mu=\delta_{\varepsilon, \varepsilon^{\prime}}
$$


for $\varepsilon, \varepsilon^{\prime} \in D$, and where $\delta_{\varepsilon, \varepsilon^{\prime}}=1$ if $\varepsilon=\varepsilon^{\prime}$ and 0 otherwise. It will be convenient for us to add the function

$$
h_{Q}^{0}=\left(\int_{Q} b d \mu\right)^{-1 / 2} \mathbf{1}_{Q},
$$

where the choice of square root is irrelevant, to get a basis of $W^{+}(Q)$. With this choice of $h_{Q}^{0}$, we'll even have (2.46) for all $\varepsilon, \varepsilon^{\prime} \in D^{+}=$ $\{0,1, \ldots, r-1\}$, because $\int_{Q} h_{Q}^{\varepsilon} b d \mu=0$ if $h_{Q}^{\varepsilon} \in W(Q)$, by (2.45). Denote by $\alpha_{\varepsilon, R} \mu(R)^{-1 / 2}$ the constant value of $h_{Q}^{\varepsilon}$ on the child $R \in$ $F(Q)$ of $Q$. Thus we want to look for $h_{Q}^{\varepsilon}$ under the form

$$
h_{Q}^{\varepsilon}=\sum_{R \in F(Q)} \alpha_{\varepsilon, R} \mu(R)^{-1 / 2} \mathbf{1}_{R} .
$$

We have already decided that

$$
\alpha_{0, R}=\left(\int_{Q} b d \mu\right)^{-1 / 2} \mu(R)^{1 / 2} .
$$

Set $b_{R}=\mu(R)^{-1} \int_{R} b d \mu$ for all $R \in F(Q)$. Note that these numbers are bounded and bounded away from 0 by (2.7). With all these notations, our constraints (2.46) are equivalent to

$$
\sum_{R \in F(Q)} \alpha_{\varepsilon, R} \alpha_{\varepsilon^{\prime}, R} b_{R}=\delta_{\varepsilon, \varepsilon^{\prime}}, \quad \text { for } \varepsilon, \varepsilon^{\prime} \in D^{+}
$$

Lemma 2.50. We can find complex numbers $\alpha_{\varepsilon, R}, 1 \leq \varepsilon \leq r-1$ and $R \in F(Q)$, such that (2.49) holds and $\left|\alpha_{\varepsilon, R}\right| \leq C$ for some constant $C$ that depends only on the accretivity constant in (2.6) and $C_{0}$ in (2.5).

To prove the lemma, some additional notation will be useful. Define a bilinear form $\langle\cdot, \cdot\rangle_{b}$ on $\mathbb{C}^{r}$ (indexed by the set $F(Q)$ of children of $Q$ ) by

$$
\langle v, w\rangle_{b}=\sum_{R} v_{R} w_{R} b_{R},
$$

where $v=\left(v_{R}\right)$ and $w=\left(w_{R}\right)$.

Now suppose we already chose coefficients $\alpha_{\varepsilon, R}, 0 \leq \varepsilon \leq k-1$, for some $k \in\{1, \ldots r-1\}$, in such a way that the equations in (2.49) hold for $0 \leq \varepsilon, \varepsilon^{\prime} \leq k-1$. (We already did this with $k=1$.) Call $v_{\varepsilon}$, 
$0 \leq \varepsilon \leq k-1$, the vector of $\mathbb{C}^{r}$ with coordinates $\alpha_{\varepsilon, R}, R \in F(Q)$. With our new notations,

$$
\left\langle v_{\varepsilon}, v_{\varepsilon^{\prime}}\right\rangle_{b}=\delta_{\varepsilon, \varepsilon^{\prime}}, \quad \text { for } 0 \leq \varepsilon, \varepsilon^{\prime} \leq k-1
$$

We want to define a new vector $v_{k}$. Set

$$
V=\left\{v \in \mathbb{C}^{r}:\left\langle v, v_{\varepsilon}\right\rangle=0 \text { for } 0 \leq \varepsilon \leq k-1\right\}
$$

Because $k \leq r-1, V$ is at least one-dimensional and in particular is not empty. Select a first vector $z \neq 0$ in $V$. Because the numbers $b_{R}$ are all $\neq 0$, we can find $w \in \mathbb{C}^{r}$ such that $\langle z, w\rangle_{b} \neq 0$. Since the $\left|b_{R}\right|$ are bounded from below, we can even choose $z$ and $w$ with bounded coefficients, and with $\langle z, w\rangle_{b}=1$.

We want to modify $w$ to get a vector in $V$. Set

$$
v=w-\sum_{\varepsilon \leq k-1}\left\langle w, v_{\varepsilon}\right\rangle_{b} v_{\varepsilon}
$$

Then

$$
\left\langle v, v_{\varepsilon^{\prime}}\right\rangle_{b}=\left\langle w, v_{\varepsilon^{\prime}}\right\rangle_{b}-\sum_{\varepsilon}\left\langle w, v_{\varepsilon}\right\rangle_{b}\left\langle v_{\varepsilon}, v_{\varepsilon^{\prime}}\right\rangle_{b}=0
$$

for all $\varepsilon^{\prime} \leq k-1$, because of (2.51). Hence $v \in V$, as desired. Also,

$$
\langle z, v\rangle_{b}=\langle z, w\rangle_{b}-\sum_{\varepsilon \leq k-1}\left\langle w, v_{\varepsilon}\right\rangle_{b}\left\langle z, v_{\varepsilon}\right\rangle_{b}=\langle z, w\rangle_{b}=1
$$

because $z \in V$.

Choose among $z, v$, and $z+v$ the vector $x$ for which $\left|\langle x, x\rangle_{b}\right|$ is largest. Note that if $\left|\langle z, z\rangle_{b}\right|$ and $\left|\langle v, v\rangle_{b}\right|$ are less than $1 / 2$, then

$$
\left|\langle z+v, z+v\rangle_{b}\right|=\left|\langle z, z\rangle_{b}+\langle v, v\rangle_{b}+2\langle z, w\rangle_{b}\right| \geq 1,
$$

by (2.55), so that $\left|\langle x, x\rangle_{b}\right| \geq 1 / 2$ in all cases. We take

$$
v_{k}=\left(\langle x, x\rangle_{b}\right)^{-1 / 2} x .
$$

It is easy to see that $v_{k}$ has coefficients $\alpha_{k, R}, R \in F(Q)$, that can be bounded in terms of the $\left|\alpha_{\varepsilon, R^{\prime}}\right|, \varepsilon \leq k-1$ and $R^{\prime} \in F(Q)$, and 
the accretivity constant for $b$. With this choice of $v_{k}$, we now have the identities in (2.49) for $\varepsilon, \varepsilon^{\prime} \leq k$. The lemma follows by induction.

Let us choose the coefficients $\alpha_{\varepsilon, R}$ as in Lemma 2.50. This defines functions $h_{Q}^{\varepsilon}, \varepsilon \in D=D(Q)$, that lie in $W(Q)$ and satisfy (2.46). Set

$$
\langle f, g\rangle_{b}=\int f g b d \mu, \quad \text { for } f, g \in L^{2}(d \mu) .
$$

With this notation, (2.46) is the same as

$$
\left\langle h_{Q}^{\varepsilon}, h_{Q}^{\varepsilon^{\prime}}\right\rangle_{b}=\delta_{\varepsilon, \varepsilon^{\prime}}, \quad \text { for } \varepsilon, \varepsilon^{\prime} \in D(Q) .
$$

Lemma 2.58. The functions $h_{Q}^{\varepsilon}, \varepsilon \in D(Q)$, form a basis of $W(Q)$, and

$$
f=\sum_{\varepsilon \in D(Q)}\left\langle f, h_{Q}^{\varepsilon}\right\rangle_{b} h_{Q}^{\varepsilon}, \quad \text { for all } f \in W(Q)
$$

In addition,

$$
C^{-1}\|f\|_{2}^{2} \leq \sum_{\varepsilon \in D(Q)}\left|\left\langle f, h_{Q}^{\varepsilon}\right\rangle_{b}\right|^{2} \leq C\|f\|_{2}^{2}
$$

for all $f \in W(Q)$, with a constant $C$ that depends only on the constants in (2.5) and (2.6).

Indeed, if $f \in W(Q)$ can be written as $f=\sum_{\varepsilon \in D} c_{\varepsilon} h_{Q}^{\varepsilon}$, then

$$
\left\langle f, h_{Q}^{\varepsilon}\right\rangle_{b}=\sum_{\varepsilon^{\prime}} c_{\varepsilon^{\prime}}\left\langle h_{Q}^{\varepsilon^{\prime}}, h_{Q}^{\varepsilon}\right\rangle_{b}=c_{\varepsilon}
$$

by (2.57). Applying this with $f=0$ gives the independence of the functions $h_{Q}^{\varepsilon}$; we then deduce that they form a basis of $W(Q)$ because we know that dimension $(W(Q))=r-1$. Thus all $f \in W(Q)$ can be written as $f=\sum_{\varepsilon \in D} c_{\varepsilon} h_{Q}^{\varepsilon}$, and the computation above shows that the $c_{\varepsilon}$ are as in (2.59).

From the formula (2.48) and the fact that the coefficients $\alpha_{\varepsilon, R}$ are bounded, we deduce at one that

$$
\left|h_{Q}^{\varepsilon}\right| \leq C \sum_{R \in F(Q)} \mu(R)^{-1 / 2} \mathbf{1}_{R}
$$


In particular,

$$
\left\|h_{Q}^{\varepsilon}\right\|_{2} \leq C
$$

If $f \in W(Q)$, then (2.59) implies that

$$
\|f\|_{2} \leq \sum_{\varepsilon \in D}\left|\left\langle f, h_{Q}^{\varepsilon}\right\rangle_{b}\right|\left\|h_{Q}^{\varepsilon}\right\|_{2} \leq C\left(\sum_{\varepsilon \in D}\left|\left\langle f, h_{Q}^{\varepsilon}\right\rangle_{b}\right|^{2}\right)^{1 / 2}
$$

by the equivalence of the $\ell^{1}$ and $\ell^{2}$-norms in $\mathbb{C}^{r-1}$, and the fact that $r \leq C_{0}$. Similarly,

$$
\sum_{\varepsilon \in D}\left|\left\langle f, h_{Q}^{\varepsilon}\right\rangle_{b}\right|^{2} \leq C_{0}\|f\|_{2}^{2}
$$

by Schwarz and (2.62). This completes our proof of Lemma 2.58.

Proposition 2.63. Every function $f \in L^{2}(d \mu)$ can be written as

$$
f=F_{0} f+\sum_{k \geq 0} \sum_{Q \in \Delta_{k}} \sum_{\varepsilon \in D(Q)}\left\langle f, h_{Q}^{\varepsilon}\right\rangle_{b} h_{Q}^{\varepsilon},
$$

where

$$
\left\langle f, h_{Q}^{\varepsilon}\right\rangle_{b}=\int_{Q} f h_{Q}^{\varepsilon} b d \mu
$$

is as in (2.56), and the convergence of the series in $k$ occurs in $L^{2}(d \mu)$. Moreover,

$$
C^{-1}\|f\|_{2}^{2} \leq\left\|F_{0} f\right\|_{2}^{2}+\sum_{k \geq 0} \sum_{Q \in \Delta_{k}} \sum_{\varepsilon \in D(Q)}\left|\left\langle f, h_{Q}^{\varepsilon}\right\rangle_{b}\right|^{2} \leq C\|f\|_{2}^{2}
$$

Finally, the decomposition in (2.64) is unique: if there is a decomposition

$$
f=f_{0}+\sum_{k} \sum_{Q \in \Delta_{k}} \sum_{\varepsilon \in D(Q)} c_{Q}^{\varepsilon} h_{Q}^{\varepsilon}
$$

where $f_{0}$ is constant on each cube of $\Delta_{0}$ and the series (in $k$ ) converges in $L^{2}(d \mu)$, then $f_{0}=F_{0} f$ and $c_{Q}^{\varepsilon}=\left\langle f, h_{Q}^{\varepsilon}\right\rangle_{b}$ for all $Q \in \bigcup_{k} \Delta_{k}$ and $\varepsilon \in D(Q)$. 
Recall from (2.10) that $F_{0}$ is a harmless projection onto the subspace of functions that are constant on each cube of $\Delta_{0}$.

We start with the proof of the existence of the decomposition and the estimate (2.66). We already have a decomposition of $f$ as $f=$ $F_{0} f+\sum_{k} Z_{k} f$, with a control on the norms, that comes from Lemma 2.33. Because of this, it will be enough to show that for all $k \geq 0$,

$$
Z_{k} f=\sum_{Q \in \Delta_{k}} \sum_{\varepsilon \in D(Q)}\left\langle f, h_{Q}^{\varepsilon}\right\rangle_{b} h_{Q}^{\varepsilon}
$$

and

$$
\left\|Z_{k} f\right\|_{2}^{2} \sim \sum_{Q \in \Delta_{k}} \sum_{\varepsilon \in D(Q)}\left|\left\langle f, h_{Q}^{\varepsilon}\right\rangle_{b}\right|^{2} .
$$

Obviously, $Z_{k} f=\sum_{Q \in \Delta_{k}} Z_{k}^{Q} f$, where $Z_{k}^{Q} f=\mathbf{1}_{Q} Z_{k} f$, and

$$
\left\|Z_{k} f\right\|_{2}^{2}=\sum_{Q \in \Delta_{k}}\left\|Z_{k}^{Q} f\right\|_{2}^{2}
$$

Thus it is enough to show that

$$
Z_{k}^{Q} f=\sum_{\varepsilon \in D(Q)}\left\langle f, h_{Q}^{\varepsilon}\right\rangle_{b} h_{Q}^{\varepsilon}
$$

and

$$
\left\|Z_{k}^{Q} f\right\|_{2}^{2} \sim \sum_{\varepsilon \in D(Q)}\left|\left\langle f, h_{Q}^{\varepsilon}\right\rangle_{b}\right|^{2}
$$

for each cube $Q \in \Delta_{k}$, and with constants in (2.71) that do not depend on $f, k$, or $Q$. In view of Lemma 2.58 , it is enough to show that $Z_{k}^{Q} f \in W(Q)$ and that

$$
\left\langle Z_{k}^{Q} f, h_{Q}^{\varepsilon}\right\rangle_{b}=\left\langle f, h_{Q}^{\varepsilon}\right\rangle_{b}
$$

for all $\varepsilon \in D(Q)$.

It is clear that $Z_{k}^{Q} f=\mathbf{1}_{Q}\left(F_{k+1} f-F_{k} f\right)$ is supported on $Q$ and constant on each child of $Q$. (See the definitions (2.10) and (2.11).) 
Also, $\int_{Q}\left(Z_{k}^{Q} f\right) b d \mu=0$ by $(2.16)$, and hence $Z_{k}^{Q} f \in W(Q)$. (See near (2.45) for the definition of $W(Q)$.) Finally, let $\varepsilon \in D(Q)$ be given. Then

$$
\begin{aligned}
\left\langle Z_{k}^{Q} f, h_{Q}^{\varepsilon}\right\rangle_{b} & =\int_{Q}\left(Z_{k}^{Q} f\right) h_{Q}^{\varepsilon} b d \mu \\
& =\int_{Q}\left(F_{k+1} f-F_{k} f\right) h_{Q}^{\varepsilon} b d \mu \\
& =\int_{Q}\left(F_{k+1} f\right) h_{Q}^{\varepsilon} b d \mu
\end{aligned}
$$

by definitions (and in particular $(2.11)$ ), the fact that $F_{k} f$ is constant on $Q$, and because

$$
\int_{Q} h_{Q}^{\varepsilon} b d \mu=0, \quad \text { for all } Q \text { and } \varepsilon \in D(Q)
$$

(because $h_{Q}^{\varepsilon} \in W(Q)$ ). Next $h_{Q}^{\varepsilon}$ is constant on each cube of $\Delta_{k+1}$, and so (2.12) (applied with $k+1$ ) tells us that

$$
\int_{Q}\left(F_{k+1} f\right) h_{Q}^{\varepsilon} b d \mu=\int_{Q} f h_{Q}^{\varepsilon} b d \mu=\left\langle f, h_{Q}^{\varepsilon}\right\rangle_{b} .
$$

This completes the proof of (2.64)-(2.66), and we are left with the uniqueness result to prove. To this effect, let us first check that

$$
\left\langle h_{Q}^{\varepsilon}, h_{Q^{\prime}}^{\varepsilon^{\prime}}\right\rangle_{b}=\delta_{(Q, \varepsilon),\left(Q^{\prime}, \varepsilon^{\prime}\right)}
$$

(that is, 1 if $Q=Q^{\prime}$ and $\varepsilon=\varepsilon^{\prime}$ and 0 otherwise) for all choices of $Q$, $Q^{\prime} \in \bigcup_{k} \Delta_{k}, \varepsilon \in D(Q)$, and $\varepsilon^{\prime} \in D\left(Q^{\prime}\right)$.

We already know this when $Q=Q^{\prime}$. When $Q$ and $Q^{\prime}$ both lie in a same $\Delta_{k}$ but $Q \neq Q^{\prime}$, then (2.75) holds because $h_{Q}^{\varepsilon}$ and $h_{Q}^{\varepsilon^{\prime}}$ have disjoint supports. Finally assume that $Q \in \Delta_{k}$ and $Q^{\prime} \in \Delta_{\ell}$, and that $\ell<k$. Then $h_{Q^{\prime}}^{\varepsilon^{\prime}}$ is constant on $Q$ and $\left\langle h_{Q}^{\varepsilon}, h_{Q}^{\varepsilon^{\prime}}\right\rangle_{b}=0$ by (2.74). Thus $(2.75)$ holds in all cases.

Now let $f \in L^{2}(d \mu)$, and suppose that $f$ has a decomposition (2.67) as in the proposition. For each choice of $Q^{\prime} \in \bigcup_{k} \Delta_{k}$ and $\varepsilon^{\prime} \in D\left(Q^{\prime}\right)$, $\left\langle f_{0}, h_{Q^{\prime}}^{\varepsilon^{\prime}}\right\rangle_{b}=0$ by $(2.74)$ and because $f_{0}$ is constant on $Q^{\prime}$. Then (2.75) tells us that

$$
\left\langle f_{0}+\sum_{k=0}^{\ell} \sum_{Q \in \Delta_{k}} \sum_{\varepsilon \in D(Q)} c_{Q}^{\varepsilon} h_{Q}^{\varepsilon}, h_{Q^{\prime}}^{\varepsilon^{\prime}}\right\rangle_{b}=c_{Q^{\prime}}^{\varepsilon^{\prime}}
$$


for $\ell$ large enough. Thus $c_{Q^{\prime}}^{\varepsilon^{\prime}}=\left\langle f, h_{Q^{\prime}}^{\varepsilon^{\prime}}\right\rangle_{b}$ by taking limits. A comparison of (2.67) with (2.64) now gives that $f_{0}=F_{0} f$ because we know that the series are the same.

This completes our proof of Proposition 2.63.

\section{A $T(b)$-theorem.}

Let $E$ be a compact subset of the plane, and let $\mu$ be a finite positive Borel measure, with support $(\mu)=E$. We shall assume that

$$
\mu(B(x, r)) \leq C_{0} r, \quad \text { for all } x \in E \text { and } r>0
$$

and some constant $C_{0}>0$. We want to state (and later prove) a $\mathrm{T}(\mathrm{b})$ theorem on the space $(E, d \mu)$ for one-dimensional singular integral operators; unfortunately, our statement will already require the existence of a collection of "dyadic cubes on $E$ " with properties somewhat stronger than those of Section 2. We shall assume that $E$ is equipped with collections $\Delta_{k}, k \geq 0$, of Borel subsets (which we'll call cubes) with the following properties.

First we ask for the same combinatorial properties as in (2.1) and $(2.2)$ :

$$
\begin{aligned}
& \text { for each } k \geq 0, E \text { is the disjoint union } \\
& \text { of the cubes } Q, Q \in \Delta_{k}, \\
& \text { if } k<\ell, Q \in \Delta_{k} \text { and } R \in \Delta_{\ell}, \\
& \text { then either } Q \cap R=\varnothing \text { or else } R \subset Q \text {. }
\end{aligned}
$$

We also require that for each integer $k \geq 0$ and each $Q \in \Delta_{k}$, there be a ball $B(Q)=B(x(Q), r(Q))$ centered on $E$ and such that

$$
A^{-k} \leq r(Q) \leq C_{1} A^{-k}
$$

and

$$
E \cap B(Q) \subset Q \subset E \cap(30 B(Q)),
$$

where $30 B(Q)=B(x(Q), 30 r(Q))$. Here $A$ and $C_{1}$ are positive constant, and we shall assume (mostly for security reasons) that $A>$ $10^{4} C_{1}$. It will be convenient for us to demand also that

$\Delta_{0}$ has only one element, 
because it will make some of the algebra easier. This is also easy to arrange, because $E$ is bounded and we could always add a first generation of cubes with only one element, or group all the cubes of $\Delta_{0}$ into a single one. (This would make the constants $C_{1}$ and $A$ slightly worse, though.)

We shall also need "small boundary" properties for our cubes. Set

$$
\begin{aligned}
N_{t}(Q)= & \left\{x \in Q: \operatorname{dist}(x, E \backslash Q) \leq t A^{-k(Q)}\right\} \\
& \cup\left\{x \in E \backslash Q: \operatorname{dist}(x, Q) \leq t A^{-k(Q)}\right\},
\end{aligned}
$$

for all $Q \in \Delta=\bigcup_{k} \Delta_{k}$ and $0<t \leq 1$, and where $k(Q)$ denotes, as in Section 2, the integer such that $Q \in \Delta_{k(Q)}$. We require the existence of an exponent $\tau \in[9 / 10,1]$ and positive numbers $\xi(Q), Q \in \Delta$, with the following properties. First,

$$
\mu\left(N_{t}(Q)\right) \leq C_{0} t^{\tau} \xi(Q), \quad \text { for all } Q \in \Delta \text { and } 0<t \leq 1 .
$$

Also,

$$
\mu(91 B(Q)) \leq C_{0} \xi(Q) \leq C_{0}^{2} A^{-k(Q)},
$$

and

$$
\sum_{\substack{R \in \Delta_{k} \\ R \subset 91 B(Q)}} \xi(R) \leq C_{0} \xi(Q),
$$

for all $k>k(Q)$. These are coherence relations that will be useful when we try to apply Shur's lemma (much later). A reasonable choice would be $\xi(Q)=\mu(92 B(Q))$, say, but this will not suffice for our application to Theorem 1.1 because we shall be working at the same time with some other measure.

Our condition (3.8) will be even more useful for cubes $Q$ such that

$$
\xi(Q) \leq C_{0} \mu(Q)
$$

Let us call these cubes good cubes. Denote by $\mathcal{G}$ the set of good cubes. We also assume that the only cube of $\Delta_{0}$ is a good cube (which would be fairly easy to arrage anyway), and add a last requirement on the numbers $\xi(Q)$ that will allow a better control on the bad cubes. We demand that

$$
\xi(Q) \leq A^{-10} \xi(\hat{Q}),
$$


whenever $Q$ is a bad cube and $\hat{Q}$ is its parent (i.e., the cube of $\Delta_{k(Q)-1}$ that contains it).

The reader may be worried by this long list of requirements. Indeed this will make it rather unpleasant to check all the hypotheses of Theorem 3.20 below, but nonetheless it is always possible to construct cubes with the properties above when $E=\operatorname{supp} \mu$ and $\mu$ satisfies (3.1). Such a construction is done in $[\mathrm{DM}]$, and we shall encounter it when we try to apply Theorem 3.20 to analytic capacity.

We shall also assume that we are given a Borel function $b$ on $E$, and that $b$ is bounded accretive, i.e., satisfies (2.6).

Now we want to describe the singular integral operators that we want to study. Denote by $\mathcal{E}$ the vector space of (finite) complex linear combinations of characteristic functions of cubes $Q \in \Delta$. Also let $b \mathcal{E}$ be the set of products $b f, f \in \mathcal{E}$. It will be easier to define our operators as operators from $b \mathcal{E}$ to its dual, or equivalently as bilinear operators from $b \mathcal{E} \times b \mathcal{E} \longrightarrow \mathbb{C}$. We shall denote by $\langle T b f, b g\rangle, f, g \in \mathcal{E}$, the effect of $T(b f)$ on $b g$ (or equivalently the image of $(b f, b g)$ under the bilinear operator). In particular, we drop the parentheses around $b f$ intentionnally, to simplify notations.

We shall assume that $T$ is associated to a "standard kernel", as follows. By standard kernel, we mean a continuous function $K(x, y)$ on $\left\{(x, y) \in \mathbb{C}^{2}: x \neq y\right\}$ such that

$$
|K(x, y)| \leq C_{2}|x-y|^{-1}, \quad \text { for } x \neq y
$$

and

$$
|K(x, y)-K(x, z)|+|K(y, x)-K(z, x)| \leq C_{2} \frac{|z-y|}{|x-y|^{2}}
$$

whenever $|z-y|<|x-y| / 2$.

The Cauchy kernel $K(x, y)=(x-y)^{-1}$ is obviously a very good example of standard kernel.

The relation between $T$ and $K$ is that

$$
\langle T f, g\rangle=\iint K(x, y) f(x) g(y) d \mu(x) d \mu(y),
$$

whenever $f, g \in b \mathcal{E}$ have disjoint supports.

By disjoint supports we mean that we can write $f$ and $g$ as $f=$ $\sum_{Q} \lambda_{Q} b \mathbf{1}_{Q}$ and $g=\sum_{R} \eta_{R} b \mathbf{1}_{R}$, with all the cubes $Q$ disjoint from 
the cubes $R$. The reader should not worry about the convergence of the integral in (3.15). We shall see later that

$$
\int_{Q} \int_{R} \frac{d \mu(x) d \mu(y)}{|x-y|}<+\infty,
$$

for all cubes $Q, R$ such that $Q \cap R=\varnothing$. This will come as a fairly easy consequence of (3.1) and (3.8), but we prefer not to check it now and try to state our main theorem soon. See (8.7) and the relevant definition (7.9) for a proof.

We shall also demand that $T$ satisfy the following analogue of the "weak boundedness property": there is a constant $C_{3} \geq 0$ such that

$$
\left|\left\langle T b \mathbf{1}_{Q}, b \mathbf{1}_{Q}\right\rangle\right| \leq C_{3} \mu(Q) \text {, for all } Q \in \Delta .
$$

Our last conditions will be that $T b \in \mathrm{BMO}$ and $T^{t} b \in \mathrm{BMO}$. Since $E$ is in general far from being a space of homogeneous type, there is some ambiguity as to which definition of BMO we should take. The following version of "dyadic-BMO" based on $L^{2}$-oscillation will be best suited to our needs.

Definition 3.18. We denote by BMO the set of functions $\beta \in L^{2}(d \mu)$ such that

$$
\int_{Q}\left|\beta(x)-m_{Q} \beta\right|^{2} d \mu(x) \leq C^{2} \mu(Q)
$$

for all cubes $Q \in \Delta$ and some $C \geq 0$.

Here

$$
m_{Q} \beta=\frac{1}{\mu(Q)} \int_{Q} \beta d \mu .
$$

We shall denote by $\|\beta\|_{\text {BMO }}$ the smallest constant $C \geq 0$ such that (3.19) holds for all $Q \in \Delta$. As usual, BMO is a Banach space of functions defined modulo an additive constant, the mean value of $\beta$ on the unique cube of $\Delta_{0}$, or equivalently the value of the constant function $E_{0} \beta$, where $E_{0}$ is as in Section 2 . We are now ready to state our $T(b)$-theorem.

Theorem 3.20. Let $E \subset \mathbb{C}$ be a compact set and $\mu$ a finite positive Borel measure such that $E=\operatorname{supp} \mu$ and (3.1) holds. Let $b$ be a bounded 
accretive function on $E$, as in (2.6). Let $\left(\Delta_{k}\right)_{k \geq 0}$ be collections of "dyadic cubes", with the properties (3.2)-(3.12). Finally let $T: b \mathcal{E} \times$ $b \mathcal{E} \longrightarrow \mathbb{C}$ be an operator that satisfies (3.13)-(3.15) and (3.17), and suppose that there are functions $\beta$ and $\tilde{\beta}$ in $\mathrm{BMO}$ such that

$$
\langle T b, b g\rangle=\int \beta b g d \mu
$$

and

$$
\langle T b g, b\rangle=\int \tilde{\beta} b g d \mu,
$$

for all $g \in \mathcal{E}$. Then $T$ extends to a bounded operator on $L^{2}(d \mu)$.

A few comments on this statement will be useful.

The conditions (3.21) and (3.22) are just a dual way to say that $T b=\beta$ and $T^{t} b=\tilde{\beta}$, where $T^{t}$ denotes the transposed operator. Recall that $\mathcal{E}$ is dense in $L^{2}(d \mu)$, as in (2.17). Since $C^{-1} \leq|b| \leq C$ by (2.6), $b \mathcal{E}$ also is dense in $L^{2}(d \mu)$ and the $\langle T b, b g\rangle, g \in \mathcal{E}$, determine $T b$.

REMARK 3.23. Because $b \mathcal{E}$ is dense in $L^{2}(d \mu)$, it is easy to see that $T$ extends to a bounded operator on $L^{2}(d \mu)$ (or, if we see $T$ as a bilinear operator, that $T$ extends to a bounded bilinear operator from $L^{2}(d \mu) \times$ $L^{2}(d \mu)$ to $\left.\mathbb{C}\right)$ if and only if there is a constant $C \geq 0$ such that

$$
|\langle T b f, b g\rangle| \leq C\|f\|_{2}\|g\|_{2}, \quad \text { for all } f, g \in \mathcal{E} .
$$

REMARK 3.25. Although this was not said explicitely in the statement, our proof will give a bound on the norm of $T$ (or equivalently on the best constant $C$ in (3.24)) that depends only $C_{0}, C_{1}, C_{2}, C_{3}, A,\|\beta\|_{\mathrm{BMO}}$ and $\|\tilde{\beta}\|_{\mathrm{BMO}}$.

Here we work with a compact set $E$, and this has the small advantage that we did not need to define $T b$ and $T^{t} b$ as "distributions modulo additive constants". Our hypothesis (3.17), applied to the only cube of $\Delta_{0}$, gives a control on the integrals of $T b$ and $T^{t} b$ against $b$ (i.e., the constant piece $F_{0}(T b)=F_{0}\left(T^{t} b\right)$, with the notations of Section 2). Thus it is not surprising that we only need to control $\|\beta\|_{\text {BMO }}$ and $\|\tilde{\beta}\|_{\text {BMO }}$ once we have (3.17). 
REMARK 3.26. As far as the main goal of this paper is concerned, the reader should not pay too much attention to the (slightly complicated) general definition of singular integral operators given here: Theorem 3.20 will be applied to operators $T_{\varepsilon}$ that can be defined brutally by integration against the very integrable kernels

$$
\frac{1}{x-y} \varphi\left(\frac{|x-y|}{\varepsilon}\right)
$$

where $\varphi$ is a smooth cut-off function that vanishes in a neighborhood of 0 . Also see the beginning of the discussion about principal value operators associated to antisymmetric standard kernels in the next section.

REMARK 3.27. In our statement we have assumed that $E=\operatorname{supp} \mu$ because this was natural and simple. However, Theorem 3.20 is still true if we only assume instead that $E$ is a bounded Borel set contained in the support of $\mu$ and such that $\mu(\mathbb{C}-E)=0$. This will not make any difference in the proof below, and it may make the hypotheses a little bit easier to check, because we could be given partitions of $E$ (rather than supp $\mu$ ) into dyadic cubes. This is not a very serious issue anyway, because it is fairly easy to see that such a partition can be extended to a partition of $\operatorname{supp} \mu$ with the same properties. See the argument a little below (3.57) in [DM].

REMARK 3.28. Our condition (3.17) is clearly necessary for $T$ to have a bounded extension to $L^{2}(d \mu)$, and we wish to claim without proof (essentially, because we shall not need this fact) that our main conditions $T b \in \mathrm{BMO}$ and $T^{t} b \in \mathrm{BMO}$ are necessary as well. The verification should amount to checking that

$$
\int_{Q}\left|T\left(\left(1-\mathbf{1}_{Q}\right) b\right)(x)-T\left(\left(1-\mathbf{1}_{Q}\right) b\right)(x(Q))\right|^{2} d \mu(x) \leq C \mu(Q),
$$

for all $Q \in \Delta$, and this would follow from

$$
\int_{Q}\left(\int_{E \backslash Q}\left|\frac{1}{x-y}-\frac{1}{x(Q)-y}\right| d \mu(y)\right)^{2} d \mu(x) \leq C \mu(Q) .
$$

We shall prove similar (only a little more complicated) estimates later; see in particular the proof of (13.65) to reduce to

$$
\int_{Q}\left(\int_{2 Q \backslash Q} \frac{d \mu(y)}{|x-y|}\right)^{2} d \mu(x)
$$


and then the proof of (13.75), where we can define $h(x)$ as in (13.70) and (13.55) but with $r(x)=0$, because $\mu$ satisfies (3.1).

REMARK 3.31. Our statement of Theorem 3.20 is clearly not optimal. We can replace our accretivity condition (2.6) with the slightly weaker requirement that $b$ be bounded and satisfy (2.7). Our choice of $\tau=$ $9 / 10$ in (3.8) is not optimal; probably a weaker definition of standard kernels would work as well and $E$ should not need to be bounded. Our hypothesis that $E$ and $K$ live in the plane (as opposed to some $\mathbb{R}^{n}$ ) is not needed (see Remark 9.112); quite possibly $E$ and $K$ do not need to be one-dimensional either. However the modifications needed to take care of all these details could be quite painful (if they exist), and our proof is already complicated enough without them. Since we only have one clear application in mind so far, it is probably wiser not to think too much about extensions now.

A more unpleasant aspect of Theorem 3.20 is that we have to use cubes with the properties (3.2)-(3.12). This will even create some trouble in the present paper, because the cubes that are given to us will come from a different measure and will not be directly adapted to the measure on which we want to apply Theorem 3.20.

It seems that F. Nazarov, S. Treil, and A. Volberg were able to prove a $T(b)$-theorem for measures that satisfy (3.1) without using our machinery with dyadic cubes [NTV]. It would be interesting to see whether their proof can be adapted to give Theorem 1.1.

In the next section we want to say a few words about the "principal value operator" associated to a given antisymmetric standard kernel. After this we'll discuss shortly how to verify that $T b$ and $T^{t} b$ lie in BMO with the help of the Haar system of Section 2.

\section{Antisymmetric standard kernels.}

Let $K$ be a standard kernel, and suppose that

$$
K(x, y)=-K(y, x), \quad \text { when } x \neq y .
$$

We want to define a singular integral operator $T: b \mathcal{E} \times b \mathcal{E} \longrightarrow \mathbb{C}$ such that (3.15) and (3.17) hold.

We start with the easy case when

$$
\int_{E \backslash\{x\}}|K(x, y)| d \mu(y) \leq C,
$$


for all $x \in E$ and some $C \geq 0$. Then we can set

$$
T f(x)=\int K(x, y) f(y) d \mu(y),
$$

for all $f \in b \mathcal{E}$ and $x \in E ; T f$ is a bounded function and

$$
\begin{aligned}
\langle T f, g\rangle & =\int T f(x) g(x) d \mu(x) \\
& =\iint K(x, y) f(y) g(x) d \mu(y) d \mu(x),
\end{aligned}
$$

with a nicely convergent integral, for all $g \in b \mathcal{E}$. By Fubini and antisymmetry,

$$
\left\langle T b \mathbf{1}_{Q}, b \mathbf{1}_{Q}\right\rangle=0, \quad \text { for all } Q \in \Delta
$$

in this case. If $f, g \in \mathcal{E}$, then for $k$ large enough we can write

$$
f=\sum_{Q \in \Delta_{k}} \lambda_{Q} \mathbf{1}_{Q} \quad \text { and } \quad g=\sum_{R \in \Delta_{k}} \eta_{R} \mathbf{1}_{R}
$$

Then (4.4) and (4.5) imply that

$$
\begin{aligned}
& \langle T b f, b g\rangle \\
& \quad=\sum_{Q, R \in \Delta_{k}} \sum_{Q \neq R} \lambda_{Q} \eta_{R} \int_{R} \int_{Q} K(x, y) b(y) b(x) d \mu(y) d \mu(x),
\end{aligned}
$$

when (4.6) holds.

When we no longer assume (4.2), the simplest is probably to get $T$ as a limit of operators $T_{\varepsilon}$, as follows. Select a nice $C^{1}$ cut-off function $\varphi$ such that $\varphi(t)=0$ for $0 \leq t \leq 1$ and $\varphi(t)=1$ for $t \geq 2$, and then set

$$
K_{\varepsilon}(x, y)=\varphi\left(\frac{|x-y|}{\varepsilon}\right) K(x, y)
$$

for all (small) $\varepsilon>0$. The kernels $K_{\varepsilon}$ are still uniformly standard and antisymmetric, and they satisfy (4.2), so we can define singular integral operators $T_{\varepsilon}$ as in the discussion above. 
Lemma 4.8. For every antisymmetric standard kernel $K$ we can define a singular integral operator $T: b \mathcal{E} \times b \mathcal{E} \longrightarrow \mathbb{C}$ by

$$
\langle T b f, b g\rangle=\lim _{\varepsilon \rightarrow 0}\left\langle T_{\varepsilon} b f, b g\right\rangle, \quad \text { for all } f, g \in \mathcal{E}
$$

Moreover T satisfies (3.15) and (4.5), and (4.7) holds whenever $f, g$ are as in (4.6).

We shall refer to $T$ as the principal value operator associated to (the antisymmetric standard kernel) $K$. Note that we shall only use (3.13), and not (3.14).

Our proof of Lemma 4.8 will rely on (3.16), which will only be proved later (see (8.7) and the definition (7.9)) but is fairly simple.

Because of (3.16), the integrals in (4.7) converge, and we could have taken (4.7) as our definition of $T$. It is slightly easier to proceed as we do because we won't have to check that different expressions for $f$ and $g$ in (4.6) give the same result in (4.7). Let us return to the lemma. The existence of a limit in (4.9) follows from the dominated convergence theorem, applied to the kernels $K_{\varepsilon}$ (that converge pointwise to $K$ ) in the formula (4.7) (which is satisfied by all the $T_{\varepsilon}$ 's as soon as (4.6) holds). We also get the formula (4.7) for $T$ at the same time. From (4.9) and the linearity of each $T_{\varepsilon}$ we get that $T$ is linear. The formula (4.5) for $T$ follows directly from (4.9) and the fact that each $T_{\varepsilon}$ satisfies it. Finally (3.15) is an easy consequence of (4.7) (and the existence of decompositions as in (4.6)), or can be obtained directly from its analogue for the $T_{\varepsilon}$ 's and the dominated convergence theorem.

This completes our discussion of the principal value operator associated to antisymmetric standard kernels. Note that they satisfy the weak boundedness property (3.17) automatically, because they satisfy the stronger (4.5).

\section{5. $T b \in \mathrm{BMO}$ and the Haar system.}

In this section we want to see how to use the modified Haar system of Section 2 to check our conditions that $T b \in$ BMO and $T^{t} b \in$ BMO.

First observe that our cubes $Q, Q \in \Delta$, satisfy the conditions (2.1)(2.5) required for the construction of Section 2: (2.1) and (2.2) are the same as (3.2) and (3.3), (2.3) follows from (3.5) and the fact that $B(Q)$ is centered on supp $\mu,(2.4)$ is a consequence of (3.4) and (3.5) (although 
with a slightly larger constant), and finally (2.5) (again with a larger constant) follows from the fact that for each $r$,

the number of cubes of $\Delta_{k}$ that meet a

ball of radius $r$ is always $\leq 1+C A^{2 k} r^{2}$.

This last is an easy consequence of (3.4), (3.5), and the fact that the balls $B(Q)$ are centered on $E$, because this implies that $\left|x(Q)-x\left(Q^{\prime}\right)\right| \geq$ $A^{-k}$ when $Q, Q^{\prime} \in \Delta_{k}$, with $Q \neq Q^{\prime}$.

So we can apply the construction of Section 2 to our cubes $Q \in \Delta$ and our function $b$. We do this and get a modified Haar system $\left\{h_{Q}^{\varepsilon}\right\}_{Q, \varepsilon}$. It will be simpler to call

$$
H=\{(Q, \varepsilon): Q \in \Delta \text { and } \varepsilon \in D(Q)\}
$$

the set of indices that show up.

For each function $\beta \in L^{2}(d \mu)$, set

$$
\beta_{Q}^{\varepsilon}=\left\langle\beta, h_{Q}^{\varepsilon}\right\rangle_{b}=\int \beta h_{Q}^{\varepsilon} b d \mu
$$

for all $(Q, \varepsilon) \in H$. These coefficients do not determine $\beta$ entirely, but only modulo the piece $F_{0} \beta$ (see (2.64) and (2.65)). Here, because $\Delta_{0}$ has only one cube, $F_{0} \beta$ is simply the constant

$$
F_{0} \beta=\left(\int_{E} b d \mu\right)^{-1} \int_{E} \beta b d \mu
$$

(See the definition (2.10).) Nonetheless, the coefficients $\beta_{Q}^{\varepsilon}$ are enough to determine whether $\beta \in \mathrm{BMO}$.

Lemma 5.5. Let $\beta \in L^{2}(d \mu)$ be given, and define the $\beta_{Q}^{\varepsilon},(Q, \varepsilon) \in H$, by (5.3). Then $\beta \in \mathrm{BMO}$ if and only if the $\beta_{Q}^{\varepsilon}$ satisfy the following quadratic Carleson measure condition: there is a constant $C \geq 0$ such that

$$
\sum_{Q \subset R} \sum_{\varepsilon \in D(Q)}\left|\beta_{Q}^{\varepsilon}\right|^{2} \leq C^{2} \mu(R), \quad \text { for all } R \in \Delta
$$

Moreover the best constant in (5.6) is equivalent to $\|\beta\|_{\mathrm{BMO}}$. 
To prove the lemma, let $\beta \in L^{2}(d \mu)$ and $R \in \Delta$ be given. Set

$$
m_{R} \beta=\frac{1}{\mu(R)} \int_{R} \beta d \mu
$$

as in Definition 3.18, and then apply Proposition 2.63 to $f=(\beta-$ $\left.m_{R} \beta\right) \mathbf{1}_{R}$. For all cubes $Q \subset R$ and all $\varepsilon \in D(Q)$,

$$
\left\langle f, h_{Q}^{\varepsilon}\right\rangle_{b}=\int_{Q} f h_{Q}^{\varepsilon} b d \mu=\beta_{Q}^{\varepsilon}
$$

(the extra term $\int_{Q} m_{R} \beta h_{Q}^{\varepsilon} b d \mu$ disappears because of (2.74)). Then

$$
\sum_{Q \subset R} \sum_{\varepsilon \in D(Q)}\left|\beta_{Q}^{\varepsilon}\right|^{2} \leq C\|f\|_{2}^{2} \leq C \int_{R}\left|\beta-m_{R} \beta\right|^{2} d \mu
$$

by the second half of (2.66).

Denote by $\lambda$ the constant value on $R$ of $F_{0} \beta+\sum_{Q, \varepsilon}\left\langle\beta, h_{Q}^{\varepsilon}\right\rangle_{b} h_{Q}^{\varepsilon}$, where the sum is restricted to the pairs $(Q, \varepsilon)$ such that $Q$ contains $R$ and is of a generation $k(Q)<k(R)$. It would be easy to check that $\lambda$ is the value of $F_{k(R)} \beta$ on $R$, but we don't need this fact. Because of $(2.64)$,

$$
(\beta-\lambda) \mathbf{1}_{R}=\sum_{Q \subset R} \sum_{\varepsilon \in D(Q)}\left\langle\beta, h_{Q}^{\varepsilon}\right\rangle_{b} h_{Q}^{\varepsilon}
$$

Apply the uniqueness result in Proposition 2.63, and then (2.66), to the function $(\beta-\lambda) \mathbf{1}_{R}$. This gives

$$
\int_{R}|\beta-\lambda|^{2} d \mu \leq C \sum_{Q \subset R} \sum_{\varepsilon \in D(Q)}\left|\beta_{Q}^{\varepsilon}\right|^{2}
$$

(recall (5.3)). Finally observe that

$$
\int_{R}\left|\beta-m_{R} \beta\right|^{2} d \mu \leq \int_{R}|\beta-\lambda|^{2} d \mu
$$

This would be true for any constant $\lambda$ : it follows from the pythagorean theorem, or the fact that $m_{R} \beta$ is the orthogonal projection of $\beta$ on the vector space of constant functions in $L^{2}(R, d \mu)$. 
When we compare (5.8), (5.11), and (5.10), we find that the quantities in (5.8) are equivalent. Lemma 5.5 follows by taking the supremum over all cubes $R$.

Lemma 5.12. Let $T: b \mathcal{E} \times b \mathcal{E} \longrightarrow \mathbb{C}$ be a bilinear operator. Set

$$
\beta_{Q}^{\varepsilon}=\left\langle T b, b h_{Q}^{\varepsilon}\right\rangle
$$

and

$$
\tilde{\beta}_{Q}^{\varepsilon}=\left\langle T b h_{Q}^{\varepsilon}, b\right\rangle,
$$

for all $(Q, \varepsilon) \in H$. Then there are functions $\beta$ and $\tilde{\beta} \in \mathrm{BMO}$ such that (3.21) and (3.22) hold if and only if the sequences $\left\{\beta_{Q}^{\varepsilon}\right\}$ and $\left\{\tilde{\beta}_{Q}^{\varepsilon}\right\}$ both satisfy the Carleson condition (5.6).

Indeed if $\beta \in \mathrm{BMO}$ is such that (3.21) holds, then (3.21) with $g=h_{Q}^{\varepsilon}$ says that the numbers $\beta_{Q}^{\varepsilon}$ in (5.13) are the same as the ones in (5.3). Lemma 5.5 then gives the desired control on the $\beta_{Q}^{\varepsilon}$. Conversely, suppose that the $\beta_{Q}^{\varepsilon}$ in (5.13) satisfy (5.6). For each integer $k \geq 0$, set

$$
\beta_{k}=\sum_{Q \in \Delta_{k}} \sum_{\varepsilon \in D(Q)} \beta_{Q}^{\varepsilon} h_{Q}^{\varepsilon}
$$

Note that

$$
\left\|\sum_{k=m}^{n} \beta_{k}\right\|_{2}^{2} \leq C \sum_{k=m}^{n} \sum_{Q \in \Delta_{k}} \sum_{\varepsilon \in D(Q)}\left|\beta_{Q}^{\varepsilon}\right|^{2},
$$

by Proposition 2.63. Since the right-hand side of (5.16) tends to 0 when $m$ and $n$ tend to $\infty$ (because $\sum_{H}\left|\beta_{Q}^{\varepsilon}\right|^{2}<+\infty$, by (5.6) applied to the only cube of $\left.\Delta_{0}\right)$, the series $\sum_{k=0}^{\infty} \beta_{k}$ converges in $L^{2}(d \mu)$. Denote its limit by $\beta^{*}$. By the uniqueness part of Proposition 2.63,

$$
\left\langle\beta^{*}, h_{Q}^{\varepsilon}\right\rangle_{b}=\beta_{Q}^{\varepsilon}, \quad \text { for all }(Q, \varepsilon) \in H,
$$

and $\beta^{*} \in$ BMO by (5.6) and Lemma 5.5.

Denote by $W$ the subspace of $\mathcal{E}$ spanned by the $h_{Q}^{\varepsilon},(Q, \varepsilon) \in H$. By (5.13) and (5.17),

$$
\langle T b, b g\rangle=\left\langle\beta^{*}, g\right\rangle_{b}, \quad \text { for all } g \in W .
$$


From Proposition 2.63 and the description of $F_{0}$ in (5.4) we see that $W$ is a subspace of codimension 1 in $\mathcal{E}$ and the one-dimensional space of constant functions is a complementary space for $W$ in $\mathcal{E}$. Thus, even though (5.18) does not imply that $\beta^{*}$ satisfies (3.21), this will be easy to fix. Set

$$
\beta=\beta^{*}+\left(\int_{E} b d \mu\right)^{-1}\left(\langle T b, b\rangle-\left\langle\beta^{*}, b\right\rangle\right)
$$

(note that $\int_{E} b d \mu \neq 0$ by accretivity.) Obviously, adding a constant to $\beta^{*}$ does not modify $\left\langle\beta^{*}, g\right\rangle_{b}$ for $g \in W$, because of (2.74). Therefore (5.18) yields

$$
\langle T b, b g\rangle=\langle\beta, g\rangle_{b}=\int \beta b g d \mu,
$$

for all $g \in W$. Since we also have that

$$
\int \beta b d \mu=\langle\beta, b\rangle=\left\langle\beta^{*}, b\right\rangle+\left(\langle T b, b\rangle-\left\langle\beta^{*}, b\right\rangle\right)=\langle T b, b\rangle,
$$

by (5.19), we see that (5.20) holds for all $g \in \mathcal{E}$, i.e., (3.21) holds. Note that $\beta$ lies in BMO because $\beta^{*}$ does. This proves the converse.

The story for the transposed operator, i.e., with (3.22) and the numbers $\tilde{\beta}_{Q}^{\varepsilon}$ is the same. This completes our proof of Lemma 5.12.

The proof of Theorem 3.20 will (continue to) keep us busy for the next few sections. The argument will follow roughly the same lines as in the Coifman-Semmes or Auscher-Tchamitchian proofs of $T(b)$. See [CJS], [AT], [Da] or [My].

\section{Paraproducts.}

In this section we want to construct bounded operators $P$ such that $P b$ and $P^{t} b$ are prescribed functions in $B M O$. We shall call them paraproducts because they look like other operators that actually looked like Bony paraproducts.

In the standard situation for the regular $T(1)$-theorem, say, these operators are bounded singular integral operators, and we can use them to substract them from the operator $T$ of Theorem 3.20; this allows one to reduce to the situation where $T 1$ and $T^{t} 1$ are equal to 0 (instead of 
just lying in BMO.) Here this approach will not work brutally, because our paraproducts will have a fairly bad kernel. We shall have to use them in the following slightly more subtle way. The boundedness of these operators, which will not be so trivial because it will use Carleson's theorem, will be used to show that their matrices in the modified Haar system of Section 2 define bounded operators on $\ell^{2}(H)$. These bounded matrices will then be substracted from the matrices of operators $T$ from Theorem 3.20, and we shall be able to prove that the resulting differences of matrices are small enough to be handled by just looking at the size of their coefficients.

In this section we construct the paraproducts, prove their boundedness, and compute their matrices. For the results of this section, none of the small boundary conditions on our cubes will be used: the weaker structure of Section 2 is still enough.

For each sequence $\left\{\beta_{Q}^{\varepsilon}\right\}_{(Q, \varepsilon) \in H}$ of complex numbers that satisfies the Carleson condition (5.6) we define an operator $P$ on $\mathcal{E}$ by

$$
P f=\sum_{(Q, \varepsilon) \in H} \beta_{Q}^{\varepsilon}\left\langle f, h_{Q}^{\varepsilon}\right\rangle_{b} \theta_{Q},
$$

where

$$
\theta_{Q}=\left(\int_{Q} b d \mu\right)^{-1} \mathbf{1}_{Q}
$$

The sum in (6.1) has only finitely many terms, because only finitely many coefficients $\left\langle f, h_{Q}^{\varepsilon}\right\rangle_{b}$ can be different from 0 when $f \in \mathcal{E}$. Thus (6.1) makes sense, and even $P f \in \mathcal{E}$.

We shall also be interested in the operator $\tilde{P}$ that we get from $P$ by "b-transposition", as follows: $\tilde{P}$ is the linear operator from $\mathcal{E}$ to the dual of $b \mathcal{E}$ defined by

$$
\langle\tilde{P} g, b f\rangle=\langle P f, b g\rangle
$$

or equivalently

$$
\langle\tilde{P} g, f\rangle_{b}=\langle P f, g\rangle_{b}, \quad \text { for all } f, g \in \mathcal{E} .
$$

Lemma 6.5. The operator $\tilde{P}$ is also given by

$$
\tilde{P} g=\sum_{(Q, \varepsilon) \in H} \beta_{Q}^{\varepsilon}\left(\int_{Q} b d \mu\right)^{-1}\left(\int_{Q} g b d \mu\right) h_{Q}^{\varepsilon},
$$


for all $g \in \mathcal{E}$ and where the series in (6.6) converges in $L^{2}(d \mu)$.

Let $g \in \mathcal{E}$ be given, and set

$$
c_{Q}^{\varepsilon}=\beta_{Q}^{\varepsilon}\left(\int_{Q} b d \mu\right)^{-1}\left(\int_{Q} g b d \mu\right) .
$$

By the paraaccretivity conditions (2.6),

$$
\left|\int_{Q} b d \mu\right|^{-1} \leq C \mu(Q)^{-1}
$$

and, since $g$ is obviously bounded, $\left|c_{Q}^{\varepsilon}\right| \leq C\left|\beta_{Q}^{\varepsilon}\right|$ for all $Q$ and $\varepsilon$. The constant $C$ may depend wildly on $g$, but we don't care. In particular, $\sum_{Q, \varepsilon}\left|c_{Q}^{\varepsilon}\right|^{2}<+\infty$ by (5.6), and the same argument as in Lemma 5.12 (see around (5.15)) shows that the series in (6.6) converges in $L^{2}(d \mu)$. Call $h \in L^{2}(d \mu)$ the limit; we want to check that $h$ can be taken as $\tilde{P} g$, i.e., that

$$
\langle h, f\rangle_{b}=\langle P f, g\rangle_{b}, \quad \text { for all } f \in \mathcal{E} .
$$

When $f$ is a constant, $\langle h, f\rangle_{b}=0$ because $h$ is a limit in $L^{2}$ of finite linear combinations of functions $h_{Q}^{\varepsilon}$ and $\left\langle h_{Q}^{\varepsilon}, f\right\rangle_{b}=0$ by (2.74). Since $P f=0$ because all the $\left\langle f, h_{Q}^{\varepsilon}\right\rangle_{b}$ are equal to 0 , we get (6.8) for constant functions. Since all functions in $\mathcal{E}$ are linear combinations of some constant and functions $h_{Q}^{\varepsilon}$ (by Proposition 2.63 and (5.4)), it is enough to prove (6.8) when $f=h_{Q}^{\varepsilon}$. But

$$
\left\langle P h_{Q}^{\varepsilon}, g\right\rangle_{b}=\beta_{Q}^{\varepsilon}\left\langle\theta_{Q}, g\right\rangle_{b}=\beta_{Q}^{\varepsilon}\left(\int_{Q} b d \mu\right)^{-1}\left(\int_{Q} g b d \mu\right)=\left\langle h, h_{Q}^{\varepsilon}\right\rangle_{b},
$$

by (6.1) $(2.75),(6.2)$, the definition of $h$ as the right-hand side of (6.6), and (2.75) again. This proves Lemma 6.5.

Proposition 6.9. The operators $P$ and $\tilde{P}$ both extend to bounded operators on $L^{2}(d \mu)$, with norms less than $C^{\prime}$ times the constant $C$ in the Carleson condition (5.6).

First observe that $P$ extends to a bounded operator on $L^{2}(d \mu)$ if and only if there is a constant $C \geq 0$ such that

$$
|\langle P f, b g\rangle| \leq C\|f\|_{2}\|g\|_{2}, \quad \text { for all } f, g \in \mathcal{E} \text {. }
$$


This follows easily from the density of $\mathcal{E}$ in $L^{2}(d \mu)$ and the fact that $C^{-1} \leq|b| \leq C$ by (2.6). This condition is also equivalent to the existence of an extension of $\tilde{P}$ to a bounded operator on $L^{2}(d \mu)$, because of (6.3). Thus it will be enough to prove the boundedness of (an extension of) $\tilde{P}$ to $L^{2}(d \mu)$.

From Lemma 6.5, the uniqueness result in Proposition 2.63, and (2.66) we deduce that for every $g \in \mathcal{E}$,

$$
\|\tilde{P} g\|_{2}^{2} \leq C \sum_{(Q, \varepsilon) \in H}\left|c_{Q}^{\varepsilon}\right|^{2}
$$

where $c_{Q}^{\varepsilon}$ is as in (6.7). We want to use Lemma 2.23 (Carleson's theorem) to estimate the right-hand side of (6.11). Set

$$
f_{k}=\sum_{Q \in \Delta_{k}} \mu(Q)^{-1}\left(\int_{Q}|g| d \mu\right) \mathbf{1}_{Q},
$$

for all $k \geq 0$. Obviously the sequence $\left\{f_{k}\right\}$ satisfies (2.24) with $f$ replaced with $g$. Also define measures $\nu_{k}$ on $E$ by

$$
d \nu_{k}=\sum_{Q \in \Delta_{k}}\left(\sum_{\varepsilon \in D(Q)}\left|\beta_{Q}^{\varepsilon}\right|^{2}\right) \mu(Q)^{-1} \mathbf{1}_{Q} d \mu .
$$

Let us check that $\left\{\nu_{k}\right\}_{k \geq 0}$ defines a Carleson measure on $E \times \mathbb{N}$, as in Definition 2.21. For each cube $R \in \bigcup_{k} \Delta_{k}$,

$$
\sum_{k \geq k(R)} \nu_{k}(R)=\sum_{Q \subset R}\left(\sum_{\varepsilon \in D(Q)}\left|\beta_{Q}^{\varepsilon}\right|^{2}\right) \leq C \mu(R),
$$

by (5.6). In other words, (2.22) holds and $\nu=\left\{\nu_{k}\right\}$ is a Carleson measure. Lemma 2.23 now tells us that

$$
\sum_{k} \int\left|f_{k}\right|^{2} d \nu_{k} \leq C\|g\|_{2}^{2} .
$$

But

$$
\begin{aligned}
\sum_{k} \int\left|f_{k}\right|^{2} d \nu_{k} & =\sum_{k} \sum_{Q \in \Delta_{k}}\left(\sum_{\varepsilon \in D(Q)}\left|\beta_{Q}^{\varepsilon}\right|^{2}\right) \mu(Q)^{-2}\left(\int_{Q}|g| d \mu\right)^{2} \\
& \geq C^{-1} \sum_{(Q, \varepsilon) \in H}\left|c_{Q}^{\varepsilon}\right|^{2}
\end{aligned}
$$


by definitions (6.12) and (6.13), the accretivity condition (2.6), and (6.7). Because of $(6.11)$, this gives that $\|\tilde{P} g\|_{2}^{2} \leq C\|g\|_{2}^{2}$, proves the boundedness of $\tilde{P}$, and completes our proof of Proposition 6.9.

Next we want to talk about matrices.

Definition 6.16. Let $T: \mathcal{E} \times b \mathcal{E} \longrightarrow \mathbb{C}$ be a bilinear operator. The matrix of $T$ (relative to the system $\left\{h_{Q}^{\varepsilon}\right\}$ ) is the matrix $\mathcal{M}$ with coefficients

$$
M\left(Q, \varepsilon, R, \varepsilon^{\prime}\right)=\left\langle T h_{Q}^{\varepsilon}, b h_{R}^{\varepsilon^{\prime}}\right\rangle, \quad(Q, \varepsilon) \in H \text { and }\left(R, \varepsilon^{\prime}\right) \in H
$$

The slight asymmetry of this definition cannot be a serious problem because $C^{-1} \leq|b| \leq C$ by (2.6); our definition is just more convenient for our paraproducts $P$ and $\tilde{P}$. Note in particular that if $\tilde{T}$ denotes the $b$-transpose of $T$ as in (6.3), i.e., if $\tilde{T}: \mathcal{E} \times b \mathcal{E} \longrightarrow \mathbb{C}$ is defined by

$$
\langle\tilde{T} g, b f\rangle=\langle T f, b g\rangle, \quad \text { for } f, g \in \mathcal{E},
$$

then the matrix of $\tilde{T}$ is just the transpose of $\mathcal{M}$.

We do not claim that $\mathcal{M}$ determines $T$, and indeed it does not say anything about $\langle T 1, b f\rangle$ or $\langle T f, b\rangle$ when $f \in \mathcal{E}$, but it will still be useful to determine when $T$ has a bounded extension to $L^{2}(d \mu)$.

Lemma 6.19. Let $T: \mathcal{E} \times b \mathcal{E} \longrightarrow \mathbb{C}$ be a bilinear operator and $\mathcal{M}$ denote its matrix relative to the system $\left\{h_{Q}^{\varepsilon}\right\}$. Then $T$ admits an extension to a bounded operator on $L^{2}(d \mu)$ if and only if

$$
\begin{aligned}
& T 1 \in L^{2}(d \mu), \\
& \tilde{T} 1 \in L^{2}(d \mu),
\end{aligned}
$$

and

$\mathcal{M}$ defines a bounded operator on $\ell^{2}(H)$.

Let us explain these conditions; (6.20) means that there is a function $h \in L^{2}(d \mu)$ such that

$$
\langle T 1, b f\rangle=\langle h, b f\rangle=\int h b f d \mu, \quad \text { for all } f \in \mathcal{E} .
$$


Similarly, (6.21) means that there is an $\tilde{h} \in L^{2}(d \mu)$ such that

$$
\langle\tilde{T} 1, b f\rangle=\langle T f, b\rangle=\int \tilde{h} b f d \mu, \quad \text { for all } f \in \mathcal{E} .
$$

As for (6.22), let $W^{*}$ denote the set of finitely supported sequences $x=\left\{x_{Q}^{\varepsilon}\right\}_{(Q, \varepsilon) \in H}$ and define a bilinear operator $S$ from $W^{*} \times W^{*}$ to $\mathbb{C}$ by

$$
\langle S x, y\rangle=\sum_{(Q, \varepsilon) \in H} \sum_{\left(R, \varepsilon^{\prime}\right) \in H} M\left(Q, \varepsilon, R, \varepsilon^{\prime}\right) x_{Q}^{\varepsilon} y_{R}^{\varepsilon^{\prime}}
$$

for all $x, y \in W^{*}$. Then (6.22) means that there is a constant $C \geq 0$ such that

$$
|\langle S x, y\rangle| \leq C\|x\|\|y\|, \quad \text { for } x, y \in W^{*},
$$

where

$$
\|x\|=\left(\sum_{(Q, \varepsilon) \in H}\left|x_{Q}^{\varepsilon}\right|^{2}\right)^{1 / 2}
$$

and similarly for $y$.

The obvious mapping from $W^{*}$ to $W=\operatorname{span}\left\{h_{Q}^{\varepsilon}:(Q, \varepsilon) \in H\right\}$ defined by $\varphi(x)=\sum x_{Q}^{\varepsilon} h_{Q}^{\varepsilon}$ is a bijection and

$$
C^{-1}\|x\| \leq\|\varphi(x)\|_{2} \leq C\|x\|,
$$

by Proposition 2.63. From (6.17) and (6.25) we deduce that

$$
\langle S x, y\rangle=\langle T \varphi(x), b \varphi(y)\rangle, \quad \text { for all } x, y \in W^{*} .
$$

Hence (6.22) holds if and only if there is a constant $C \geq 0$ such that

$$
|\langle T f, b g\rangle| \leq C\|f\|_{2}\|g\|_{2}, \quad \text { for all } f, g \in W .
$$

Because of this, (6.22) is clearly necessary if we want $T$ to have a bounded extension; (6.20) and (6.21) are necessary too, because $1 \in$ $L^{2}(d \mu)$ and $T$ has a bounded extension if and only if $\tilde{T}$ does. The converse is not much harder. Suppose that (6.20), (6.21), and (6.22) hold. By Proposition 2.63, every $f \in \mathcal{E}$ has a decomposition $f=$ $F_{0} f+\pi f$, where $F_{0} f$ is a constant because $\Delta_{0}$ has only one cube, $\pi f \in W$, and

$$
\left\|F_{0} f\right\|_{2}+\|\pi f\|_{2} \leq C\|f\|_{2} .
$$


Then, for $f, g \in \mathcal{E}$,

$$
\begin{aligned}
|\langle T f, b g\rangle| & \leq\left|\left\langle T\left(F_{0} f\right), b g\right\rangle\right|+|\langle T(\pi f), b g\rangle| \\
& \leq C\left|F_{0} f\right|\|T 1\|_{2}\|g\|_{2}+\left|\left\langle T(\pi f), b F_{0} g\right\rangle\right|+|\langle T(\pi f), b \pi g\rangle| \\
& \leq C\left\|F_{0} f\right\|_{2}\|g\|_{2}+C\left|F_{0} g\right|\|\tilde{T} 1\|_{2}\|\pi f\|_{2}+C\|\pi f\|_{2}\|\pi g\|_{2} \\
& \leq C\left\|F_{0} f\right\|_{2}\|g\|_{2}+C\left\|F_{0} g\right\|_{2}\|f\|_{2}+C\|f\|_{2}\|g\|_{2} \\
& \leq C\|f\|_{2}\|g\|_{2}
\end{aligned}
$$

by $(6.20),(6.21)$, and (6.28). Thus $T$ has a bounded extension to $L^{2}$, as desired.

This completes the proof of Lemma 6.19.

Finally we want to compute the matrix of $P$.

Lemma 6.30. Denote by $\mathcal{P}=\left(\left(P\left(Q, \varepsilon, R, \varepsilon^{\prime}\right)\right)\right)$ the matrix of the paraproduct $P$ defined by (6.1) (using the sequence $\left\{\beta_{Q}^{\varepsilon}\right\}$.) Then

$$
P\left(Q, \varepsilon, R, \varepsilon^{\prime}\right)=0, \quad \text { when } Q \cap R=\varnothing \text { or } R \subset Q \text {, }
$$

and

$$
\begin{aligned}
& P\left(Q, \varepsilon, R, \varepsilon^{\prime}\right) \text { is } \beta_{Q}^{\varepsilon} \text { times the constant value } \\
& \text { of } h_{R}^{\varepsilon^{\prime}} \text { on } Q \text { when } Q \subset R, Q \neq R .
\end{aligned}
$$

Recall from (6.17) and (6.1) that

$$
P\left(Q, \varepsilon, R, \varepsilon^{\prime}\right)=\left\langle P h_{Q}^{\varepsilon}, b h_{R}^{\varepsilon^{\prime}}\right\rangle=\beta_{Q}^{\varepsilon}\left\langle\theta_{Q}, b h_{R}^{\varepsilon^{\prime}}\right\rangle,
$$

by (2.75). This is obviously 0 when $Q \cap R=\varnothing$, and also when $R \subset Q$ because $\theta_{Q}$ is constant on $Q$, and by (2.74). Thus we are left with the case when $Q \subset R, Q \neq R$. In this case $h_{R}^{\varepsilon^{\prime}}$ is constant on $Q$ and

$$
\left\langle\theta_{Q}, b\right\rangle=\int \theta_{Q} b d \mu=1
$$

by (6.2). The lemma follows. 


\section{Reduction to the study of a matrix $\mathcal{N}$.}

In this section we take an operator $T$ that satisfies the hypotheses of Theorem 3.20, compute its matrix, substract from it the matrices of appropriate paraproducts, and show that the remaining matrix defines a bounded operator if some other matrix $\mathcal{N}$ defines a bounded operator on $\ell^{2}$. The matrix $\mathcal{N}$ will be a matrix with nonnegative coefficients, that no longer depends on the operator $T$ but only on the size of certain integrals on $E$. The boundedness of (the operator defined by) $\mathcal{N}$ will be proved in later sections, with the help of Schur's lemma.

We shall not use the small boundary properties of our cubes in this section either, except for the fact that

$$
\mu(\{x \in Q: \operatorname{dist}(x, E \backslash Q)\})=0, \quad \text { for all } Q \in \Delta,
$$

which follows from (3.8).

Let $T$ be an operator that satisfies the hypotheses of Theorem 3.20. Denote by $\mathcal{T}=\left(\left(T\left(Q, \varepsilon, R, \varepsilon^{\prime}\right)\right)\right)$ the matrix of $T M_{b}$ in the modified Haar system $\left\{h_{Q}^{\varepsilon}\right\}$, and where $M_{b}$ denotes the operator of pointwise multiplication by $b$. Since $T$ is defined on $b \mathcal{E} \times b \mathcal{E}, T M_{b}$ is defined on $\mathcal{E} \times b \mathcal{E}$, as required in Definition 6.16 , and

$$
T\left(Q, \varepsilon, R, \varepsilon^{\prime}\right)=\left\langle T b h_{Q}^{\varepsilon}, b h_{R}^{\varepsilon^{\prime}}\right\rangle, \quad \text { for }(Q, \varepsilon),\left(R, \varepsilon^{\prime}\right) \in H .
$$

We already know from $(3.21)$ and $(3.22)$ that $\left(T M_{b}\right) 1=\beta$ and $\left(\tilde{T} M_{b}\right) 1=$ $\tilde{\beta}$ lie in BMO, hence in $L^{2}(d \mu)$. (Compare (3.21) and (3.22) with (6.23) and (6.24) for $T M_{b}$.) Hence Lemma 6.19 says that it will be enough to prove that $\mathcal{T}$ defines a bounded operator on $\ell^{2}(H)$.

Next define sequences $\left\{\beta_{Q}^{\varepsilon}\right\}$ and $\left\{\tilde{\beta}_{Q}^{\varepsilon}\right\}$ by (5.13) and (5.14). Then Lemma 5.12 says that $\left\{\beta_{Q}^{\varepsilon}\right\}$ and $\left\{\tilde{\beta}_{Q}^{\varepsilon}\right\}$ satisfy the Carleson condition (5.6).

Denote by $P$ the paraproduct constructed in Section 6 with the sequence $\left\{\beta_{Q}^{\varepsilon}\right\}$ and by $P^{*}$ the analogous operator defined with the sequence $\left\{\tilde{\beta}_{Q}^{\varepsilon}\right\}$. These two operators have bounded extensions to $L^{2}(d \mu)$, by Proposition 6.9. Denote by $\mathcal{P}$ the matrix of $P$. By Lemma 6.19, $\mathcal{P}$ defines a bounded operator on $\ell^{2}(H)$, and so does its transpose $\tilde{\mathcal{P}}$. Similarly, the matrix $\mathcal{P}^{*}$ of $P^{*}$ defines a bounded operator on $\ell^{2}(H)$.

Set $\mathcal{M}=\mathcal{T}-\tilde{\mathcal{P}}-\mathcal{P}^{*}$ and denote by $M\left(Q, \varepsilon, R, \varepsilon^{\prime}\right)$ its generic element. The discussion above shows that

Theorem 3.20 will follow if we can prove that

$\mathcal{M}$ defines a bounded operator on $\ell^{2}(H)$. 
Let us compute the coefficients of $\mathcal{M}$. We use (7.2), Lemma 6.30, and then (5.14) and (5.13) to get that

$$
M\left(Q, \varepsilon, R, \varepsilon^{\prime}\right)=\left\langle T b h_{Q}^{\varepsilon}, b h_{R}^{\varepsilon^{\prime}}\right\rangle,
$$

when $Q \cap R=\varnothing$ or $Q=R$,

$$
\begin{aligned}
M\left(Q, \varepsilon, R, \varepsilon^{\prime}\right) & =\left\langle T b h_{Q}^{\varepsilon}, b h_{R}^{\varepsilon^{\prime}}\right\rangle-P^{*}\left(Q, \varepsilon, R, \varepsilon^{\prime}\right) \\
& =\left\langle T b h_{Q}^{\varepsilon}, b h_{R}^{\varepsilon^{\prime}}\right\rangle-\tilde{\beta}_{Q}^{\varepsilon}\left(\text { value of } h_{R}^{\varepsilon^{\prime}} \text { on } Q\right) \\
& =\left\langle T b h_{Q}^{\varepsilon}, b h_{R}^{\varepsilon^{\prime}}\right\rangle-\left\langle T b h_{Q}^{\varepsilon}, b\right\rangle\left(\text { value of } h_{R}^{\varepsilon^{\prime}} \text { on } Q\right),
\end{aligned}
$$

when $Q \subset R, Q \neq R$, and

$$
\begin{aligned}
M\left(Q, \varepsilon, R, \varepsilon^{\prime}\right) & =\left\langle T b h_{Q}^{\varepsilon}, b h_{R}^{\varepsilon^{\prime}}\right\rangle-P\left(R, \varepsilon^{\prime}, Q, \varepsilon\right) \\
& =\left\langle T b h_{Q}^{\varepsilon}, b h_{R}^{\varepsilon^{\prime}}\right\rangle-\beta_{R}^{\varepsilon^{\prime}}\left(\text { value of } h_{Q}^{\varepsilon} \text { on } R\right) \\
& =\left\langle T b h_{Q}^{\varepsilon}, b h_{R}^{\varepsilon^{\prime}}\right\rangle-\left\langle T b, b h_{R}^{\varepsilon^{\prime}}\right\rangle\left(\text { value of } h_{Q}^{\varepsilon} \text { on } R\right),
\end{aligned}
$$

when $R \subset Q, R \neq Q$.

The next stage of our computation is to express the coefficients of $\mathcal{M}$ in terms of the kernel $K(x, y)$ and then estimate them in terms of some integrals on $E$. The following notation will be useful. Set

$$
d(Q)=A^{-k(Q)},
$$

for all $Q \in \Delta$, where $k(Q)$ denotes the generation of $Q$, and also

$$
2 Q=\{x \in E: \operatorname{dist}(x, Q) \leq d(Q)\} \text {. }
$$

For each Borel subset $V$ of $E$ such that $Q \cap V=\varnothing$, set

$$
I(Q, V)=\int_{V} \int_{Q} \frac{d \mu(x) d \mu(y)}{|x-y|},
$$

and

$$
J(Q, V)=\int_{V} \frac{d(Q) d \mu(x)}{|x-x(Q)|^{2}}
$$

where $x(Q)$ denotes the center of the ball $B(Q)$, as in (3.5). These are the quantities that will be used to control the coefficients of $\mathcal{M}$. We 
still denote by $F(Q), Q \in \Delta$, the set of children of $Q$, i.e., the set of cubes $Q^{*} \in \Delta_{k(Q)+1}$ such that $Q^{*} \subset Q$. We shall try to be systematic about calling $Q^{*}$ or $R^{*}$ generic children of $Q$ or $R$.

Lemma 7.11. If $Q \cap R=\varnothing$, then for all choices of $\varepsilon \in D(Q)$ and $\varepsilon^{\prime} \in D(R)$,

$$
\left|M\left(Q, \varepsilon, R, \varepsilon^{\prime}\right)\right| \leq C A_{1}(Q, R)+C A_{2}(Q, R),
$$

where

$$
\begin{aligned}
& A_{1}(Q, R) \\
& \quad=\sum_{Q^{*} \in F(Q)} \sum_{R^{*} \in F(R)} \mu\left(Q^{*}\right)^{-1 / 2} \mu\left(R^{*}\right)^{-1 / 2} I\left(Q^{*}, R^{*} \cap 2 Q\right)
\end{aligned}
$$

and

$$
A_{2}(Q, R)=\mu(Q)^{1 / 2} \sum_{R^{*} \in F(R)} \mu\left(R^{*}\right)^{-1 / 2} J\left(Q, R^{*} \backslash 2 Q\right)
$$

To prove the lemma, let us first observe that $T b h_{Q}^{\varepsilon}(x)$ is welldefined when $\operatorname{dist}(x, Q)>0$ and that it is given by

$$
T b h_{Q}^{\varepsilon}(x)=\int_{Q} K(x, y) b(y) h_{Q}^{\varepsilon}(y) d \mu(y) .
$$

Recall from (2.48) and Lemma 2.50 that

$$
h_{Q}^{\varepsilon}=\sum_{Q^{*} \in F(Q)} \alpha_{\varepsilon, Q^{*}} \mu\left(Q^{*}\right)^{-1 / 2} \mathbf{1}_{Q^{*}}
$$

where the coefficients $\alpha_{\varepsilon, Q^{*}}$ are uniformly bounded. From this description and the first standard estimate (3.13) we get that

$$
\left|T b h_{Q}^{\varepsilon}(x)\right| \leq C \sum_{Q^{*} \in F(Q)} \mu\left(Q^{*}\right)^{-1 / 2} \int_{Q^{*}} \frac{d \mu(y)}{|x-y|},
$$

when $\operatorname{dist}(x, Q)>0$. Notice incidentally that $\operatorname{dist}(x, Q)>0$ for $\mu$ almost all $x \in R$, by (7.1) (or (3.8)).

This estimate is best when $x \in 2 Q \backslash Q$, but when $x \notin 2 Q$ we can use the second standard estimate (3.14) and the fact that $\int_{Q} b h_{Q}^{\varepsilon} d \mu=0$ 
(by (2.74)) to get a better one. Let $x(Q)$ denote the center of $B(Q)$, as usual. (Actually, for the computation that follows, any point of $Q$ would work equally well.) If $x \in E \backslash 2 Q$,

$$
\begin{aligned}
\left|T b h_{Q}^{\varepsilon}(x)\right| & =\left|\int_{Q} K(x, y) b(y) h_{Q}^{\varepsilon}(y) d \mu(y)\right| \\
& =\left|\int_{Q}(K(x, y)-K(x, x(Q))) b(y) h_{Q}^{\varepsilon}(y) d \mu(y)\right| \\
& \leq C \int_{Q} \frac{|y-x(Q)|}{|x-x(Q)|^{2}}\left|b(y) h_{Q}^{\varepsilon}(y)\right| d \mu(y) \\
& \leq C \frac{d(Q)}{|x-x(Q)|^{2}} \sum_{Q^{*} \in F(Q)} \mu\left(Q^{*}\right)^{1 / 2} \\
& \leq C \mu(Q)^{1 / 2} \frac{d(Q)}{|x-x(Q)|^{2}},
\end{aligned}
$$

by $(7.15),(3.5),(3.4)$ and (7.16). We may now use (3.15), (7.4), (7.16) and the discussion above to get that

$$
\begin{aligned}
\left|M\left(Q, \varepsilon, R, \varepsilon^{\prime}\right)\right| & =\left|\left\langle T b h_{Q}^{\varepsilon}, b h_{R}^{\varepsilon^{\prime}}\right\rangle\right| \\
& \leq C \sum_{R^{*} \in F(R)} \mu\left(R^{*}\right)^{-1 / 2} \int_{R^{*}}\left|T b h_{Q}^{\varepsilon}(x)\right| d \mu(x) .
\end{aligned}
$$

On each $R^{*} \cap 2 Q$ we use (7.17) to estimate $\left|T b h_{Q}^{\varepsilon}(x)\right|$; when we integrate the estimate and sum over $R^{*}$, we get less than $C A_{1}(Q, R)$. Similarly, we use (7.18) for $x \in R^{*} \backslash 2 Q$, integrate over $R^{*} \backslash 2 Q$ and sum over $R^{*}$, and we get a contribution $\leq C A_{2}(Q, R)$. This proves Lemma 7.11.

Note that our estimate is more performant when $d(Q) \leq d(R)$; in the other situations, we would use a symmetric argument. We won't need to do this, because as we shall see soon we won't have to bound coefficients of $\mathcal{M}$ for which $d(Q)>d(R)$.

Lemma 7.20. We have that

$$
\left|M\left(Q, \varepsilon, Q, \varepsilon^{\prime}\right)\right| \leq C+C A_{3}(Q),
$$

for all $Q \in \Delta$ and $\varepsilon, \varepsilon^{\prime} \in D(Q)$, where

$$
A_{3}(Q)=\sum_{Q_{1}^{*} \in F(Q)} \sum_{\substack{Q_{2}^{*} \in F(Q) \\ Q_{2}^{*} \neq Q_{1}^{*}}} \mu\left(Q_{1}^{*}\right)^{-1 / 2} \mu\left(Q_{2}^{*}\right)^{-1 / 2} I\left(Q_{1}^{*}, Q_{2}^{*}\right) .
$$


To prove the lemma we start again from (7.4) and use (7.16) to get that

$$
\begin{aligned}
& M\left(Q, \varepsilon, Q, \varepsilon^{\prime}\right)=\left\langle T b h_{Q}^{\varepsilon}, b h_{Q}^{\varepsilon^{\prime}}\right\rangle \\
&= \sum_{Q_{1}^{*}} \sum_{Q_{2}^{*} \in F(Q)} \alpha_{\varepsilon, Q_{1}^{*}} \alpha_{\varepsilon^{\prime}, Q_{2}^{*}} \mu\left(Q_{1}^{*}\right)^{-1 / 2} \mu\left(Q_{2}^{*}\right)^{-1 / 2} \\
& \cdot\left\langle T b \mathbf{1}_{Q_{1}^{*}}, \mathbf{1}_{Q_{2}^{*}}\right\rangle .
\end{aligned}
$$

The terms for which $Q_{1}^{*}=Q_{2}^{*}$ are less or equal than $C C_{3}$, by our weak boundedness assumption (3.17), and so we are left with terms for which $Q_{1}^{*} \neq Q_{2}^{*}$. For each such term we use (3.15) and (3.13) to get that

$$
\begin{aligned}
\left|\left\langle T b \mathbf{1}_{Q_{1}^{*}}, b \mathbf{1}_{Q_{2}^{*}}\right\rangle\right| & =\left|\int_{Q_{1}^{*}} \int_{Q_{2}^{*}} K(x, y) b(y) b(x) d \mu(y) d \mu(x)\right| \\
& \leq C I\left(Q_{1}^{*}, Q_{2}^{*}\right) .
\end{aligned}
$$

Lemma 7.20 follows because the coefficients $\alpha_{Q, \varepsilon}$ are uniformly bounded.

Now we want to estimate the coefficients of $\mathcal{M}$ for which $Q \subset R$, $Q \neq R$. In such situations, we shall systematically denote by $R(Q)$ the child of $R$ that contains $Q$.

Lemma 7.25. For each choice of cubes $Q \subset R, Q \neq R$ and $\varepsilon \in D(Q)$, $\varepsilon^{\prime} \in D(R)$,

$$
\left|M\left(Q, \varepsilon, R, \varepsilon^{\prime}\right)\right| \leq C\left(B_{11}+B_{12}+B_{21}+B_{22}\right),
$$

where

$$
\begin{gathered}
B_{11}=\sum_{Q^{*} \in F(Q)} \sum_{\substack{R^{*} \in F(R) \\
R^{*} \neq R(Q)}} \mu\left(Q^{*}\right)^{-1 / 2} \mu\left(R^{*}\right)^{-1 / 2} \\
B_{12}=\sum_{\substack{R^{*} \in F(R) \\
R^{*} \neq R(Q)}} \mu(Q)^{1 / 2} \mu\left(R^{*}\right)^{-1 / 2} J\left(Q, R^{*} \cap 2 Q\right), \\
B_{21}=\sum_{Q^{*} \in F(Q)} \mu\left(Q^{*}\right)^{-1 / 2} \mu(R(Q))^{-1 / 2} I\left(Q^{*}, 2 Q \backslash R(Q)\right),
\end{gathered}
$$


and

$$
B_{22}=\mu(Q)^{1 / 2} \mu(R(Q))^{-1 / 2} J(Q, E \backslash(2 Q \cup R(Q)))
$$

To prove the lemma, let $Q, \varepsilon, R, \varepsilon^{\prime}$ be given, and denote by $\alpha$ the constant value of $h_{R}^{\varepsilon^{\prime}}$ on $Q$. Thus $|\alpha| \leq C \mu(R(Q))^{-1 / 2}$ by (7.16). This time we apply (7.5)

$$
M\left(Q, \varepsilon, R, \varepsilon^{\prime}\right)=\left\langle T b h_{Q}^{\varepsilon}, b h_{R}^{\varepsilon^{\prime}}\right\rangle-\alpha\left\langle T b h_{Q}^{\varepsilon}, b\right\rangle=B_{1}-B_{2},
$$

where

$$
B_{1}=\left\langle T b h_{Q}^{\varepsilon}, b h_{R}^{\varepsilon^{\prime}} \mathbf{1}_{R \backslash R(Q)}\right\rangle
$$

and

$$
B_{2}=\alpha\left\langle T b h_{Q}^{\varepsilon}, \mathbf{1}_{E-R(Q)} b\right\rangle .
$$

Note that the part $\left\langle T b h_{Q}^{\varepsilon}, \alpha \mathbf{1}_{R(Q)} b\right\rangle$ cancelled out; this will allow us to use the kernel $K(x, y)$ again to estimate $B_{1}$ and $B_{2}$. Thus

$$
\left|B_{1}\right| \leq C \sum_{\substack{R^{*} \in F(R) \\ R^{*} \neq R(Q)}} \mu\left(R^{*}\right)^{-1 / 2} \int_{R^{*}}\left|T b h_{Q}^{\varepsilon}(x)\right| d \mu(x),
$$

by (3.15) and (7.16) for $R$, and now we can estimate $\left|T b h_{Q}^{\varepsilon}(x)\right|$ with (7.17) and (7.18). As before, we use (7.17) on each $R^{*} \cap 2 Q$. After we integrate on $R^{*} \cap 2 Q$ and sum over $R^{*}$, we get a contribution less or equal than $C B_{11}$. On the rest of $R^{*}$ we use (7.18), and we get a total contribution less or equal than $C B_{12}$ after integrating on $R^{*} \backslash 2 Q$ and summing over $R^{*}$.

The estimates for $B_{2}$ are similar. Recall that $|\alpha| \leq C \mu(R(Q))^{-1 / 2}$ and hence

$$
\left|B_{2}\right| \leq C \mu(R(Q))^{-1 / 2} \int_{E \backslash R(Q)}\left|T b h_{Q}^{\varepsilon}(x)\right| d \mu(x) .
$$

On $2 Q \backslash R(Q)$ we use (7.17) and get a contribution less or equal than $C B_{21}$. On $E \backslash(2 Q \cup R(Q))$ we use (7.18) and get less or equal than $C B_{22}$. This proves Lemma 7.25. 
We are now ready to reduce the proof of Theorem 3.20 to the "verification" that a certain matrix $\mathcal{N}$ defines a bounded operator on $\ell^{2}(\Delta)$. Define a matrix $\mathcal{N}=((N(Q, R)))_{Q, R \in \Delta}$ as follows. Set

$$
N(Q, R)=A_{1}(Q, R)+A_{2}(Q, R),
$$

where $A_{1}(Q, R)$ and $A_{2}(Q, R)$ are as in (7.13) and (7.14), when

$$
\begin{gathered}
Q \cap R=\varnothing \text { and either } d(Q)<d(R) \text { or else } \\
d(Q)=d(R) \text { and } \operatorname{diam} Q \leq \operatorname{diam} R, \\
N(Q, Q)=A_{3}(Q), \quad \text { for } Q \in \Delta,
\end{gathered}
$$

and

$$
N(Q, R)=B_{11}+B_{12}+B_{21}+B_{22},
$$

when $Q \subset R, Q \neq R$, where $A_{3}(Q)$ is as in (7.22) and the $B_{i j}$ are as in Lemma 7.25. Finally set $N(Q, R)=0$ in the other cases, i.e., when $Q \cap R=\varnothing$ but (7.37) does not hold and when $R \subset Q, R \neq Q$.

Lemma 7.40. To prove Theorem 3.20 it is enough to show that $\mathcal{N}$ defines a bounded operator on $\ell^{2}(\Delta)$.

Set $\mathcal{N}^{+}=\mathcal{N}+\mathcal{N}^{t}+\operatorname{Id}$, where $\mathcal{N}^{t}$ is the transpose of $\mathcal{N}$ and Id the identity matrix. Obviously $\mathcal{N}^{+}$defines a bounded operator on $\ell^{2}(\Delta)$ if $\mathcal{N}$ does. Let us suppose that this is the case; since $\mathcal{N}^{+}$is a matrix with nonnegative entries and all the sets $D(Q), Q \in \Delta$, have at most $C$ elements, we shall get that $\mathcal{M}$ defines a bounded operator on $\ell^{2}(H)$ if we can prove that

$$
\left|M\left(Q, \varepsilon, R, \varepsilon^{\prime}\right)\right| \leq N^{+}(Q, R)
$$

for all $Q, \varepsilon, R, \varepsilon^{\prime}$, and where $N^{+}(Q, R)$ denotes the generic element of $\mathcal{N}^{+}$.

Denote by $D_{0}$ the set of (ordered) pairs $(Q, R)$ such that $Q \subset R$ or (7.37) holds. When $(Q, R) \in D_{0},(7.41)$ follows from Lemma 7.11, 7.20 , or 7.25. Otherwise, we shall use the transpose $\tilde{T}$ of $T$, which is defined by $\langle\tilde{T} b f, b g\rangle=\langle T b g, b f\rangle$ for all $f, g \in \mathcal{E}$. Notice that $\tilde{T}$ also satisfies the hypotheses of Theorem 3.20 , only with $K(x, y)$ replaced with $K(y, x)$ and the functions $\beta, \tilde{\beta}$ exchanged. We can define a matrix 
$\tilde{\mathcal{M}}$ with $\tilde{T}$ as we did for $T$ itself, and it is clear from (7.4)-(7.6) that $\tilde{\mathcal{M}}$ is the transpose of $\mathcal{M}$. If $(Q, R) \notin D_{0}$, then $(R, Q) \in D_{0}$ and

$$
\left|M\left(Q, \varepsilon, R, \varepsilon^{\prime}\right)\right|=\left|\tilde{M}\left(R, \varepsilon^{\prime}, Q, \varepsilon\right)\right| \leq N^{+}(R, Q)=N^{+}(Q, R),
$$

by Lemma $7.11,7.20$ or 7.25 (applied to $\tilde{T}$.) Thus (7.41) holds in all cases, and $\mathcal{M}$ defines a bounded operator if $\mathcal{N}$ does. Lemma 7.40 follows, by (7.3).

We completed the task assigned to this section: we can forget singular integral operators and concentrate on the matrix $\mathcal{N}$.

\section{Estimates on $I(Q, V)$.}

We shall need to estimate the various coefficients of our new matrix $\mathcal{N}$. In this section we prove a few estimates on integrals like $I(Q, V)$ that will be useful later. The small boundary properties (3.8)-(3.12) will be needed here.

We start with a simple estimate that uses the density property (3.1) only. First observe that

$$
\begin{aligned}
\int_{|x-y| \geq d} \frac{d \mu(y)}{|x-y|^{2}} & \leq \sum_{\ell \geq 0} \int_{2^{\ell} d \leq|x-y|<2^{\ell+1} d} \frac{d \mu(y)}{|x-y|^{2}} \\
& \leq C \sum_{\ell \geq 0}\left(2^{\ell} d\right)\left(2^{\ell} d\right)^{-2} \\
& \leq C d^{-1}
\end{aligned}
$$

for all $x \in E$ and $d>0$.

Next let $Q \in \Delta$ and $V \subset E \backslash Q$ be given. For each $x \in Q$ we use Cauchy-Schwarz to show that

$$
\int_{V} \frac{d \mu(y)}{|x-y|} \leq \mu(V)^{1 / 2}\left(\int_{V} \frac{d \mu(y)}{|x-y|^{2}}\right)^{1 / 2} \leq C \mu(V)^{1 / 2} d(x)^{-1 / 2}
$$

where we set $d(x)=\operatorname{dist}(x, E \backslash Q)$. Note that $d(x)>0$ almost everywhere on $Q$, by (3.7)-(3.8). We may now integrate (8.2) on $Q$ to get that

$$
I(Q, V)=\int_{Q} \int_{V} \frac{d \mu(y) d \mu(x)}{|x-y|} \leq C \mu(V)^{1 / 2} \int_{Q} d(x)^{-1 / 2} d \mu(x)
$$


(see (7.9) for the definition of $I(Q, V)$ ).

Lemma 8.4. We have that

$$
\int_{Q} d(x)^{-1 / 2} d \mu(x) \leq C d(Q)^{-1 / 2} \xi(Q)
$$

Here $d(Q)=A^{-k(Q)}$, as in (7.7). To prove the lemma we decompose $Q$ into a first region $B_{0}$ where $d(x) \geq d(Q)$ and annuli $B_{\ell}, \ell \geq 1$, where $2^{-\ell} d(Q) \leq d(x)<2^{-\ell+1} d(Q)$. Then

$$
\int_{B_{0}} d(x)^{-1 / 2} d \mu(x) \leq d(Q)^{-1 / 2} \mu(Q) \leq C d(Q)^{-1 / 2} \xi(Q),
$$

by (3.9), and

$$
\begin{aligned}
\int_{B_{\ell}} d(x)^{-1 / 2} d \mu(x) & \leq 2^{\ell / 2} d(Q)^{-1 / 2} \mu\left(B_{\ell}\right) \\
& \leq C 2^{\ell / 2} d(Q)^{-1 / 2} 2^{-\tau \ell} \xi(Q),
\end{aligned}
$$

for $\ell \geq 1$, by (3.8). Lemma 8.4 follows by summing a convergent power series.

From (8.3) and Lemma 8.4 we deduce that

$$
I(Q, V) \leq C \mu(V)^{1 / 2} \xi(Q) d(Q)^{-1 / 2},
$$

for all cubes $Q$ and all sets $V \subset E \backslash Q$.

We want to refine this estimate when $Q$ is not a good cube (as in (3.11)), because getting estimates in terms of $\mu(Q)$ rather than $\xi(Q)$ will be very useful to get rid of some of the negative powers in formulae like (7.13), (7.22), (7.27) or (7.29). Recall that $\mu$ is not doubling or anything like that, and we don't have much in terms of lower bounds for $\mu$.

Lemma 8.8. We have that

$$
I(Q, V) \leq C \mu(V)^{1 / 2} \mu(Q)^{1 / 2} \xi(Q)^{1 / 2} d(Q)^{-1 / 2},
$$

for all $Q \in \Delta$ and $V \subset E \backslash Q$. 
To prove this we shall use a decomposition of $Q_{0}$ into maximal good subcubes. For each $Q \in \Delta$, denote by $S(Q)$ the set of maximal good cubes contained in $Q$. Obviously the cubes $S, S \in S(Q)$, are disjoint and contained in $Q$, but it is also true that they almost cover $Q$, i.e., that

$$
\mu\left(Q \backslash \bigcup_{S \in S(Q)} S\right)=0 .
$$

This is essentially [DM, Lemma 5.28], but the proof is quite simple and so we give it here. For each integer $\ell>0$, let $Z_{\ell}$ denote the set of cubes $R \in \Delta_{k(Q)+\ell}$ such that $R \subset Q$ but $R$ is not contained in any $S \in S(Q)$. Such cubes are obviously bad, as well as all their ancestors until $Q$ and hence they satisfy

$$
\mu(R) \leq C_{0} \xi(R) \leq C_{0} A^{-10 \ell} \xi(Q),
$$

by (3.9) and repeated uses of (3.12). Because of (5.1) and (3.4), (3.5), $Z_{\ell}$ has at most $C A^{2 \ell}$ elements, and so

$$
\mu\left(\bigcup_{R \in Z_{\ell}} R\right) \leq C A^{-8 \ell} \xi(Q),
$$

where the value of $C$ does not matter because we only need to know that $\mu\left(\bigcup_{R \in Z_{\ell}} R\right)$ tends to 0 when $\ell \longrightarrow+\infty$. The desired estimate (8.10) follows because

$$
\left(Q \backslash \bigcup_{S(Q)} S\right) \subset\left(\bigcup_{R \in Z_{\ell}} R\right)
$$

for all $\ell>0$.

To prove Lemma 8.8 we use (8.10) to almost-decompose $Q$ into its maximal good subcubes $S, S \in S(Q)$ and write

$$
\begin{aligned}
I(Q, V) & =\int_{Q} \int_{V} \frac{d \mu(x) d \mu(y)}{|x-y|} \\
& =\sum_{S \in S(Q)} I(S, V) \\
& \leq C \mu(V)^{1 / 2} \sum_{S \in S(Q)} \xi(S) d(S)^{-1 / 2} \\
& \leq C \mu(V)^{1 / 2} \sum_{S \in S(Q)} \mu(S) d(S)^{-1 / 2}
\end{aligned}
$$


by (8.7) and (3.11) for the good cubes $S$.

Lemma 8.14. For all $Q \in \Delta$,

$$
\sum_{S \in S(Q)} \mu(S)\left(\frac{d(Q)}{d(S)}\right)^{6} \leq C \xi(Q)
$$

Of course we don't need the power 6 here, but the proof will be just as easy. Denote by $S_{\ell}(Q), \ell \geq 0$, the set of cubes $S \in S(Q)$ such that $k(S)=k(Q)+\ell$. Because of $(5.1), S_{\ell}(Q)$ has at most $C A^{2 \ell}$ elements. Let us check that

$$
\mu(S) \leq C_{0} A^{-10(\ell-1)} \xi(Q),
$$

for all $S \in S_{\ell}(Q)$. When $\ell=0$ or $1, \mu(S) \leq \mu(Q) \leq C_{0} \xi(Q)$ by (3.9). When $\ell>1, \mu(S) \leq \mu(\hat{S}) \leq C_{0} \xi(\hat{S}) \leq C_{0} A^{-10(\ell-1)} \xi(Q)$ by (3.9) and repeated uses of (3.12), and where $\hat{S}$ denotes the parent of $S$. Here we use the fact that all the ancestors of $S$ between $\hat{S}$ and $Q$ are bad, by definition of $S(Q)$.

From (8.16) and the fact that $S_{\ell}(Q)$ has at most $C A^{2 \ell}$ elements we deduce that the contribution of $S_{\ell}(Q)$ to the left-hand side of (8.15) is at most $C A^{2 \ell} A^{-10 \ell} A^{6 \ell} \xi(Q) \leq C A^{-2 \ell} \xi(Q)$; Lemma 8.14 follows by summing over $\ell \geq 0$.

Most of the time, Lemma 8.14 will be used in combination with Cauchy-Schwarz, as follows

$$
\begin{aligned}
\sum_{S \in S(Q)} \mu(S)\left(\frac{d(Q)}{d(S)}\right)^{3} & \leq\left(\sum_{S \in S(Q)} \mu(S)\right)^{1 / 2}\left(\sum_{S \in S(Q)} \mu(S)\left(\frac{d(Q)}{d(S)}\right)^{6}\right)^{1 / 2} \\
& \leq C \mu(Q)^{1 / 2} \xi(Q)^{1 / 2}
\end{aligned}
$$

because $Q$ is (essentially) the disjoint union of the cubes $S \in S(Q)$. A trivial consequence of $(8.17)$ is

$$
\begin{aligned}
\sum_{S \in S(Q)} \mu(S) d(S)^{-1 / 2} & =d(Q)^{-1 / 2} \sum_{S} \mu(S)\left(\frac{d(Q)}{d(S)}\right)^{1 / 2} \\
& \leq C d(Q)^{-1 / 2} \mu(Q)^{1 / 2} \xi(Q)^{1 / 2}
\end{aligned}
$$

Lemma 8.8 follows from this and (8.13). 
We shall need a last estimate on $I(Q, V)$, to be used when we have a larger power of $\mu(Q)$ to recuperate

$$
I(Q, 2 Q \backslash Q) \leq C \mu(Q)\left(\frac{\xi(Q)}{d(Q)}\right)^{1 / 2} .
$$

To prove this we write

$$
\begin{aligned}
I(Q, 2 Q \backslash Q) & =\sum_{S \in S(Q)} I(S, 2 Q \backslash Q) \\
& \leq \sum_{S \in S(Q)} I(S, 2 S \backslash S)+\sum_{S \in S(Q)} I(S, 2 Q \backslash 2 S) \\
& =I_{1}+I_{2} .
\end{aligned}
$$

For each $S \in S(Q)$,

$$
I(S, 2 S \backslash S) \leq C \mu(2 S)^{1 / 2} \xi(S) d(S)^{-1 / 2} \leq C \mu(S)^{3 / 2} d(S)^{-1 / 2}
$$

by (8.7), (3.9) and (3.11) for the good cube $S$. Hence

$$
\begin{aligned}
I_{1} & \leq C \sum_{S \in S(Q)} \mu(S)^{3 / 2} d(S)^{-1 / 2} \\
& \leq C \mu(Q)^{1 / 2} \sum_{S} \mu(S) d(S)^{-1 / 2} \\
& \leq C \mu(Q) \xi(Q)^{1 / 2} d(Q)^{-1 / 2},
\end{aligned}
$$

by (8.18). This takes care of $I_{1}$.

As for $I_{2}$, let us check that

$$
\int_{2 Q \backslash 2 S} \frac{d \mu(y)}{|x-y|} \leq C \frac{\xi(Q)}{d(Q)},
$$

for all $S \in S(Q)$ and $x \in S$.

Denote by $T_{\ell}, 0 \leq \ell \leq k(S)-k(Q)$, the cube of $\Delta_{k(Q)+\ell}$ that contains $S$. This is a decreasing sequence of cubes, with $T_{0}=Q$ and, $T_{k(S)-k(Q)}=S$, and $2 Q \backslash 2 S$ is the union of the sets $2 T_{\ell} \backslash 2 T_{\ell+1}, 0 \leq$ $\ell \leq k(S)-k(Q)-1$. For these values of $\ell$,

$$
\mu\left(2 T_{\ell}\right) \leq C_{0} \xi\left(T_{\ell}\right) \leq C_{0} A^{-10 \ell} \xi(Q),
$$


by (3.9) and repeated uses of (3.12). Then

$$
\begin{aligned}
\int_{2 Q \backslash 2 S} \frac{d \mu(y)}{|x-y|} & =\sum_{\ell=0}^{k(S)-k(Q)-1} \int_{2 T_{\ell} \backslash 2 T_{\ell+1}} \frac{d \mu(y)}{|x-y|} \\
& \leq \sum_{\ell} \mu\left(2 T_{\ell}\right) d\left(T_{\ell+1}\right)^{-1} \\
& \leq C \xi(Q) d(Q)^{-1}
\end{aligned}
$$

by definition (7.8) of $2 T_{\ell+1}$, the fact that $x \in S \subset T_{\ell+1}$, and then (8.24). This proves (8.23). Now

$$
\begin{aligned}
I_{2} & =\sum_{S \in S(Q)} \int_{S} \int_{2 Q \backslash 2 S} \frac{d \mu(y) d \mu(x)}{|x-y|} \\
& \leq C \sum_{S} \mu(S) \xi(Q) d(Q)^{-1} \\
& \leq C \mu(Q) \xi(Q) d(Q)^{-1} \\
& \leq C \mu(Q)\left(\frac{\xi(Q)}{d(Q)}\right)^{1 / 2}
\end{aligned}
$$

by the definitions (8.20) and (7.9), (8.23), and (3.9) (to get that $\xi(Q) \leq$ $C d(Q))$. The desired estimate (8.19) follows from (8.20), (8.22) and (8.26).

\section{Bounds on $\mathcal{N}$.}

In the original version of this paper, the matrix $\mathcal{N}$ was bounded with the help of Schur's lemma. This was quite tempting, but it turns out that it actually complicated the estimates. The current section was revisited in October 1997, after the author noticed that in the similar extension of $T(b)$ by Nazarov, Treil, and Volberg, the corresponding estimates were much simpler. Here is the simple trick that makes the difference; I am sure the reader will be glad that the authors of [NTV] kindly communicated it to me.

Lemma 9.1. Let $\mathcal{N}=((N(Q, R)))_{Q, R \in \Delta}$ be a matrix with complex coefficients. Assume that for each $Q \in \Delta$ there are at most $C_{1}$ indices 
$R \in \Delta$ such that $N(Q, R) \neq 0$, and also that

$$
\sum_{Q \in \Delta}|N(Q, R)|^{2} \leq C_{2}^{2}, \quad \text { for each } R \in \Delta
$$

Then $\mathcal{N}$ defines a bounded operator on $\ell^{2}(\Delta)$, with norm $\|\mid \mathcal{N}\| \| \leq$ $C_{1} C_{2}$.

This is easy to prove. First observe that if $\mathcal{N}$ is as in the lemma, then it is the sum of at most $C_{1}$ matrices that satisfy the hypotheses of the lemma with $C_{1}=1$ and the same constant $C_{2}$. Thus we may assume that $C_{1}=1$. For each $R \in \Delta$, denote by $v_{R} \in \ell^{2}(\Delta)$ the vector with coordinates $N(Q, R), Q \in \Delta$. By (9.2), $\left\|v_{R}\right\|^{2} \leq C_{2}^{2}$, while our first hypothesis with $C_{1}=1$ says that the vectors $v_{R}, R \in \Delta$, are orthogonal to each other. Hence if $x=\left(x_{R}\right)_{R \in \Delta}$ is any vector in $\ell^{2}(\Delta)$,

$$
\|\mathcal{N} x\|^{2}=\left\|\sum_{R} x_{R} v_{R}\right\|^{2}=\sum_{R}\left|x_{R}\right|^{2}\left\|v_{R}\right\|^{2} \leq C_{2}^{2}\|x\|^{2}
$$

as needed. The lemma follows.

To estimate the matrix $\mathcal{N}$ from Section 7 , we want to decompose it into a sum of matrices $\mathcal{N}^{k}$, with $k=k(Q)-k(R)$ and prove geometrically decreasing bounds on the norms $\left\|\left|\mathcal{N}^{k}\right|\right\|$. For each integer $k \geq 0$, denote by $\mathcal{N}^{k}$ the matrix with coefficients $N^{k}(Q, R)=N(Q, R)$ when $k(Q)=k(R)+k$ and $N^{k}(Q, R)=0$ otherwise. Note that $\mathcal{N}=\sum_{k>0} \mathcal{N}^{k}$, because $N(Q, R)=0$ when $k(Q)<k(R)$. See around (7.36)-(7.39) for the definition of $\mathcal{N}$.

At this point, and for almost all the rest of this section, we fix an integer $k \geq 0$ and we study $\mathcal{N}^{k}$ by cutting it into smaller pieces. As we shall see, Lemma 9.1 will be quite handy for most of them.

Case A. Terms with $Q=R$. Of course this only shows up when $k=$ 0 . Denote by $\mathcal{N}_{1}$ the part of $\mathcal{N}$ that lives on the diagonal, i.e., set $N_{1}(Q, R)=0$ when $Q \neq R$ and $N_{1}(Q, R)=N(Q, R)=A_{3}(Q)$ for $Q \in \Delta$. (See (7.38).)

Recall from (7.22) that

$$
A_{3}(Q)=\sum_{Q_{1}^{*}} \sum_{Q_{2}^{*}} \mu\left(Q_{1}^{*}\right)^{-1 / 2} \mu\left(Q_{2}^{*}\right)^{-1 / 2} I\left(Q_{1}^{*}, Q_{2}^{*}\right)
$$


where we sum over pairs of distinct children of $Q$. By (8.9) and (3.9),

$$
\begin{aligned}
I\left(Q_{1}^{*}, Q_{2}^{*}\right) & \leq C \mu\left(Q_{2}^{*}\right)^{1 / 2} \mu\left(Q_{1}^{*}\right)^{1 / 2} \xi\left(Q_{1}^{*}\right)^{1 / 2} d\left(Q_{1}^{*}\right)^{-1 / 2} \\
& \leq C \mu\left(Q_{2}^{*}\right)^{1 / 2} \mu\left(Q_{1}^{*}\right)^{1 / 2}
\end{aligned}
$$

and so $A_{3}(Q)$ is a sum of boundedly many bounded terms. Thus $\mathcal{N}_{1}$ defines a bounded operator on $\ell^{2}(\Delta)$, with norm ||$\left|\mathcal{N}_{1}\right| \| \leq C$.

Case B. Terms coming from $A_{1}(Q, R)$. Set $N_{2}(Q, R)=A_{1}(Q, R)$ when $k(Q)=k(R)+k$ and (7.37) holds, and $N_{2}(Q, R)=0$ otherwise. We should perhaps have written $N_{2}^{k}(Q, R)$ instead of $N_{2}(Q, R)$, but $k$ is fixed and we'll try to keep the notation simple. Note that $N_{2}(Q, R)=0$ unless $2 Q$ meets $R$; this is clear from the definitions (7.13) and (7.9). Thus for each $Q$ there are at most $C$ cubes $R \in \Delta_{k(Q)-k}$ such that $N_{2}(Q, R) \neq 0$. We can apply Lemma 9.1 to the matrix $\mathcal{N}_{2}$ with coefficients $\mathcal{N}_{2}(Q, R)$ and get that

$$
\left\|\left|\mathcal{N}_{2}\right|\right\|^{2} \leq C \sup _{R \in \Delta} \Sigma(R)
$$

where

$$
\Sigma(R)=\sum_{Q \in \Delta(R)} N_{2}(Q, R)^{2}
$$

and $\Delta(R)$ is the set of cubes $Q \in \Delta_{k(R)+k}$ such that $Q \cap R=\varnothing$ but $2 Q \cap R \neq \varnothing$.

Fix $R \in \Delta$, plug (7.13) into (9.7) and then apply (8.9) to get that

$$
\begin{aligned}
\Sigma(R) & \leq C \sum_{R^{*} \in F(R)} \sum_{Q \in \Delta(R)} \sum_{Q^{*} \in F(Q)} \mu\left(Q^{*}\right)^{-1} \mu\left(R^{*}\right)^{-1} I\left(Q^{*}, R^{*} \cap 2 Q\right)^{2} \\
& \leq C \sum_{R^{*}} \sum_{Q} \sum_{Q^{*}} \mu\left(R^{*}\right)^{-1} \mu\left(R^{*} \cap 2 Q\right) \xi\left(Q^{*}\right) d\left(Q^{*}\right)^{-1} .
\end{aligned}
$$

Let us fix $R^{*}$ and try to bound the corresponding sum. Let us warm up with the easy case when $k \leq 10$, say. Then we simply say that $\xi\left(Q^{*}\right) d\left(Q^{*}\right)^{-1} \leq C$ by (3.9), that the $R^{*} \cap 2 Q, Q \in \Delta_{k(R)+k}$, have bounded overlap (by (3.4), (3.5)) and are contained in $R^{*}$ and then that $\Sigma(R) \leq C$ after summing over boundedly many children $R^{*}$ of $R$.

For larger $k$ we wish to argue that since $Q \cap R=\varnothing$ by (7.37), the sets $R^{*} \cap 2 Q$ only cover a small proportion of $R^{*}$. This can be 
implemented directly if $R^{*}$ is a good cube, but in general we need to bring in the decomposition of $R^{*}$ into maximal good subcubes $S$, $S \in S\left(R^{*}\right)$, as in (8.10). For each $R^{*} \in F(R)$ set $S_{+}=\left\{S \in S\left(R^{*}\right)\right.$ : $k(S) \leq k(R)+k / 2\}, R_{+}^{*}=\bigcup_{S \in S_{+}} S$ and $R_{-}^{*}=R^{*} \backslash R_{+}^{*}$. We write

$$
\Sigma(R) \leq C \sum_{R^{*} \in F(R)}\left(\sigma_{+}\left(R^{*}\right)+\sigma_{-}\left(R^{*}\right)\right) \mu\left(R^{*}\right)^{-1}
$$

where

$$
\sigma_{ \pm}\left(R^{*}\right)=\sum_{Q \in \Delta(R)} \sum_{Q^{*} \in F(Q)} \mu\left(R_{ \pm}^{*} \cap 2 Q\right) \xi\left(Q^{*}\right) d\left(Q^{*}\right)^{-1}
$$

For $\sigma_{+}\left(R^{*}\right)$ we say that $\xi\left(Q^{*}\right) \leq C d\left(Q^{*}\right)$ by (3.9), so that

$$
\begin{aligned}
\sigma_{+}\left(R^{*}\right) & =\sum_{S \in S^{+}} \sum_{Q \in \Delta(R)} \sum_{Q^{*} \in F(Q)} \mu(S \cap 2 Q) \xi\left(Q^{*}\right) d\left(Q^{*}\right)^{-1} \\
& \leq C \sum_{S \in S^{+}} \sum_{Q \in \Delta(R)} \mu(S \cap 2 Q) \leq C \sum_{S \in S^{+}} \mu\left(A_{S}\right)
\end{aligned}
$$

where $A_{S}$ is the union of the sets $S \cap 2 Q, Q \in \Delta(R)$. We used the fact that the $2 Q, Q \in \Delta_{k(R)+k}$, have bounded overlap. Next all the points of $A_{S}$ lie within $A^{-k(R)-k}=A^{-k} d(R)$ of some point of $E \backslash S$, because the cubes $Q$ do not meet $R$ (and even less $S$ ). (See (7.8) for the definition of $2 Q$.) Hence $A_{S}$ is contained in the set $N_{t}(S)$ of (3.7), with $t=A^{-k} d(R) d(S)^{-1} \leq C A^{-k / 2}$ (because $d(R) \leq A^{k / 2} d(S)$ by definition of $\left.S_{+}\right)$. So

$$
\begin{aligned}
\sigma_{+}\left(R^{*}\right) & \leq C \sum_{S \in S_{+}} A^{-k \tau / 2} \xi(S) \\
& \leq C A^{-k \tau / 2} \sum_{S \in S_{+}} \mu(S) \\
& \leq C A^{-k \tau / 2} \mu\left(R^{*}\right)
\end{aligned}
$$

because the cubes $S$ are good (as in (3.11)), disjoint, and contained in $R^{*}$. This will be enough to take care of $S_{+}$.

For $\sigma_{-}\left(R^{*}\right)$ we only say that $\mu\left(R_{-}^{*} \cap 2 Q\right) \leq \mu\left(R^{*}\right)$, but we use a better estimate for $\xi\left(Q^{*}\right)$. Let $Q \in \Delta(R)$ be such that $2 Q$ meets $R_{-}^{*}$, and let $z$ be any point of $2 Q \cap R_{-}^{*}$. Then let $H$ be the smallest cube 
that contains $z$ such that $k(H) \leq k(R)+k / 2$. Since $H$ is not contained in any cube of $S_{+}$, it is a bad cube and so are all its ancestors contained in $R^{*}$. Then

$$
\begin{aligned}
\xi(H) & \leq A^{-10\left(k(H)-k\left(R^{*}\right)\right)} \xi\left(R^{*}\right) \\
& \leq C A^{-5 k} \xi\left(R^{*}\right) \\
& \leq C A^{-5 k} d(R),
\end{aligned}
$$

by repeated applications of (3.12), because $k(H) \geq k(R)+k / 2-1$, and by (3.9).

Since $2 Q$ meets $H$ and $Q$ is of a strictly later generation than $H, Q$ is contained in $91 B(H)$ and (3.10) says that $\xi\left(Q^{*}\right) \leq C \xi(H) \leq$ $C A^{-5 k} d(R)$ for all $Q^{*} \in F(Q)$.

Thus all the terms in the sum that defines $\sigma_{-}\left(R^{*}\right)$ (in (9.10)) are at most

$$
C \mu\left(R^{*}\right) A^{-5 k} d(R) d(Q)^{-1} \leq C \mu\left(R^{*}\right) A^{-4 k} .
$$

Since by easy geometric considerations (like (5.1)) there are at most $C A^{2 k}$ cubes $Q$ in $\Delta(R)$, we get that

$$
\sigma_{-}\left(R^{*}\right) \leq C \mu\left(R^{*}\right) A^{-2 k} .
$$

From this and the similar estimate (9.12) we deduce that $\sum(R) \leq$ $C A^{-\tau k / 2}$ (see (9.9)), and then that $\left\|\left|\mathcal{N}_{2} \|\right| \leq C A^{-\tau k / 4}\right.$ (by (9.6)).

Case C. Terms from $B_{11}$. Set $N_{3}(Q, R)=B_{11}$, where $B_{11}$ is as in (7.27), when $Q \subset R, Q \neq R$, and $k(Q)=k(R)+k$. Otherwise set $\mathcal{N}_{3}(Q, R)=0$. These coefficients are like the $\mathcal{N}_{2}(Q, R)=A_{1}(Q, R)$ that we just treated (compare (7.27) with (7.13)), except that now we sum over pairs $Q^{*}, R^{*}$ such that $Q^{*} \in F(Q)$ and $R^{*} \in F(R)$ is not the cube of $F(R)$ that contains $Q$. The same estimates as before can be carried out, because whenever we used the fact that $Q$ does not meet $R$ in subsection $B$, we only needed to know that $Q$ does not meet $R^{*}$. So the matrix $\mathcal{N}_{3}$ with coefficients $\mathcal{N}_{3}(Q, R)$ has a norm $\left\|\left|\mathcal{N}_{3} \|\right| \leq C A^{-\tau k / 4}\right.$, and the proof is the same as for $\mathcal{N}_{2}$.

Case D. Terms from $B_{21}$. Now set $N_{4}(Q, R)=B_{21}$, where $B_{21}$ is as in (7.29), when $Q \subset R, Q \neq R$, and $k(Q)=k(R)+k$. Otherwise set $N_{4}(Q, R)=0$. These coefficients are a little like the previous ones, but with a $\mu\left(R^{*}\right)^{-1 / 2}$ replaced with $\mu(R(Q))^{-1 / 2}$, where $R(Q)$ is the child 
of $R$ that contains $Q$. To accommodate this change, it will be better to use (8.19) rather than (8.9). Recall from (7.29) that

$$
B_{21}=\sum_{Q^{*} \in F(Q)} \mu\left(Q^{*}\right)^{-1 / 2} \mu(R(Q))^{-1 / 2} I\left(Q^{*}, 2 Q \backslash R(Q)\right),
$$

and note that

$$
\begin{aligned}
I\left(Q^{*}, 2 Q \backslash R(Q)\right) & \leq I\left(Q^{*}, 2 Q \backslash Q^{*}\right) \\
& \leq I\left(Q^{*}, 2 Q \backslash 2 Q^{*}\right)+I\left(Q^{*}, 2 Q^{*} \backslash Q^{*}\right),
\end{aligned}
$$

by definition of $I(\cdot)$ (see $(7.9))$.

The last term is at most

$$
C \mu\left(Q^{*}\right) \xi\left(Q^{*}\right)^{1 / 2} d\left(Q^{*}\right)^{-1 / 2} \leq C \mu\left(Q^{*}\right) \xi(Q)^{1 / 2} d(Q)^{-1 / 2}
$$

by (8.19) and (3.10).

The first term is

$$
\begin{aligned}
I\left(Q^{*}, 2 Q \backslash 2 Q^{*}\right) & \leq \mu\left(Q^{*}\right) \mu(2 Q) \operatorname{dist}\left(Q^{*}, 2 Q \backslash Q^{*}\right)^{-1} \\
& \leq C \mu\left(Q^{*}\right) \mu(2 Q) d(Q)^{-1} \\
& \leq C \mu\left(Q^{*}\right) \xi(Q) d(Q)^{-1} \\
& \leq C \mu\left(Q^{*}\right) \xi(Q)^{1 / 2} d(Q)^{-1 / 2}
\end{aligned}
$$

by (7.9) and (3.9). Thus

$$
N_{4}(Q, R)^{2} \leq C \sum_{Q^{*} \in F(Q)} \mu\left(Q^{*}\right) \mu(R(Q))^{-1} \xi(Q) d(Q)^{-1} .
$$

Note that for each $Q \in \Delta$ there is at most one cube $R \in \Delta$ such that $N_{4}(Q, R) \neq 0$ (namely, the ancestor of order $k$ of $Q$ ). Thus we can apply Lemma 9.1 to the matrix $\mathcal{N}_{4}$ with coefficients $N_{4}(Q, R)$, and

$$
\left\|\left|\mathcal{N}_{4}\right|\right\| \leq \sup _{R \in \Delta} \Sigma(R)
$$

with

$$
\begin{aligned}
\Sigma(R) & =\sum_{Q} N_{4}(Q, R)^{2} \\
& \leq C \sum_{R^{*} \in F(R)} \sum_{Q \in \Delta\left(R^{*}\right)} \sum_{Q^{*} \in F(Q)} \mu\left(Q^{*}\right) \mu\left(R^{*}\right)^{-1} \xi(Q) d(Q)^{-1},
\end{aligned}
$$


and where

$$
\Delta\left(R^{*}\right)=\left\{Q \in \Delta_{k(R)+k}: Q \subset R^{*} \text { and } 2 Q \text { meets } E \backslash R^{*}\right\} .
$$

(The last condition is needed if we want $I\left(Q^{*}, 2 Q \backslash R(Q)\right) \neq 0$ in (9.15).)

We shall now proceed as in Case B. As before, the case when $k \leq 10$ is easy, because we can just use (3.9) to get that

$$
\Sigma(R) \leq \sum_{R^{*}} \sum_{Q} \sum_{Q^{*}} \mu\left(Q^{*}\right) \mu\left(R^{*}\right)^{-1} \leq C
$$

(because the cubes $Q^{*}$ are disjoint and contained in $R^{*}$ ). So we may assume $k \geq 10$.

Set

$$
S_{+}=\left\{S \in S\left(R^{*}\right): k(S) \leq k(R)+\frac{k}{2}\right\}
$$

and subdivide $\Delta\left(R^{*}\right)$ into $\Delta^{+}$and $\Delta^{-}$, where

$$
\Delta^{+}=\left\{Q \in \Delta\left(R^{*}\right): Q \subset S \text { for some } S \in S_{+}\right\}
$$

and $\Delta^{-}=\Delta\left(R^{*}\right) \backslash \Delta^{+}$. For cubes of $\Delta^{+}$we use (3.9) to get that

$$
\begin{aligned}
\sigma_{+}\left(R^{*}\right) & =: \sum_{Q \in \Delta^{+}} \sum_{Q^{*} \in F(Q)} \mu\left(Q^{*}\right) \xi(Q) d(Q)^{-1} \\
& \leq C \sum_{Q \in \Delta^{+}} \mu(Q) \\
& \leq C \sum_{S \in S_{+}} \sum_{\substack{Q \in \Delta^{+} \\
Q \subset S}} \mu(Q) .
\end{aligned}
$$

Now for $S \in S_{+}$and $Q \in \Delta^{+}, Q \subset S$, we have that $2 Q$ meets $E \backslash R^{*}$ by definition of $\Delta\left(R^{*}\right)$ and so $Q \subset N_{t}(S)$, with $t=A^{-k(Q)+k(S)+1}$, say. By definition of $S_{+}, t \leq C A^{-k / 2}$ and so (3.8) yields

$$
\sum_{\substack{Q \subset S \\ Q \in \Delta^{+}}} \mu(Q) \leq \mu\left(N_{t}(S)\right) \leq C A^{-k \tau / 2} \xi(S) \leq C A^{-k \tau / 2} \mu(S)
$$

because $S$ is a good cube. Altogether, (9.20) becomes

$$
\sigma_{+}\left(R^{*}\right) \leq C \sum_{S \in S_{+}} A^{-k \tau / 2} \mu(S) \leq C A^{-k \tau / 2} \mu\left(R^{*}\right)
$$


because the maximal cubes $S, S \in S_{+}$, are disjoint and contained in $R^{*}$.

Next we want to estimate

$$
\sigma_{-}\left(R^{*}\right)=\sum_{Q \in \Delta^{-}} \sum_{Q^{*} \in F(Q)} \mu\left(Q^{*}\right) \xi(Q) d(Q)^{-1}
$$

This time we shall just say that $\mu\left(Q^{*}\right) \leq \mu\left(R^{*}\right)$, but we'll use a better estimate on $\xi(Q)$. By definition of $\Delta^{ \pm}$, the smallest ancestor $H$ of $Q$ such that $k(H) \leq k(R)+k / 2$ is a bad cube, and so are all its ancestors in $R^{*}$. By repeated uses of (3.12),

$$
\begin{aligned}
\xi(H) & \leq A^{-10\left(k(H)-k\left(R^{*}\right)\right)} \xi\left(R^{*}\right) \\
& \leq C A^{-5 k} \xi\left(R^{*}\right) \\
& \leq C A^{-5 k} d(R)
\end{aligned}
$$

(by (3.9)). Also, (3.10) says that $\xi(Q) \leq C_{0} \xi(H)$. Altogether,

$$
\xi(Q) d(Q)^{-1} \leq C \xi(H) d(Q)^{-1} \leq C A^{-4 k} .
$$

By (5.1), there are at most $C A^{2 k}$ cubes $Q$ in $\Delta\left(R^{*}\right)$ and so $\sigma_{-}\left(R^{*}\right) \leq$ $C A^{-2 k} \mu\left(R^{*}\right)$. Finally

$$
\Sigma(R) \leq C \sum_{R^{*} \in F(R)} \mu\left(R^{*}\right)^{-1}\left(\sigma_{+}\left(R^{*}\right)+\sigma_{-}\left(R^{*}\right)\right) \leq C A^{-k \tau / 2},
$$

by (9.19), (9.20), (9.23), (9.22) and this, and so $\left\|\left|\mathcal{N}_{4} \|\right| \leq C A^{-k \tau / 4}\right.$ by (9.18).

Case E. The far part from $A_{2}(Q, R)$. Now we study the piece of $\mathcal{N}^{k}$ that comes from terms $A_{2}(Q, R)$ for which dist $(Q, R) \geq d(R)$. For each $R \in$ $\Delta$ denote by $\mathcal{A}(R)$ the set of cubes $Q \in \Delta_{k(R)+k}$ for which (7.37) holds and $\operatorname{dist}(Q, R) \geq d(R)=A^{-k(R)}$. Define $\mathcal{N}_{5}$ by $N_{5}(Q, R)=A_{2}(Q, R)$ when $Q \in \mathcal{A}(R)$ and $N_{5}(Q, R)=0$ otherwise. When $Q \in \mathcal{A}(R)$,

$$
\begin{aligned}
A_{2}(Q, R) & =\mu(Q)^{1 / 2} \sum_{R^{*} \in F(R)} \mu\left(R^{*}\right)^{-1 / 2} J\left(Q, R^{*} \backslash 2 Q\right) \\
& \leq \mu(Q)^{1 / 2} \sum_{R^{*} \in F(R)} \mu\left(R^{*}\right)^{-1 / 2} \int_{R^{*}} \frac{d(Q) d \mu(x)}{|x-x(Q)|^{2}} \\
& \leq C \mu(Q)^{1 / 2} \sum_{R^{*}} \mu\left(R^{*}\right)^{1 / 2} d(Q) \operatorname{dist}\left(Q, R^{*}\right)^{-2} \\
& \leq C \mu(Q)^{1 / 2} \mu(R)^{1 / 2} d(Q) \operatorname{dist}(Q, R)^{-2}
\end{aligned}
$$


by (7.14) and (7.10).

Subdivide each $\mathcal{A}(R)$ further into the

$$
\mathcal{A}_{\ell}(R)=\left\{Q \in \mathcal{A}(R): 2^{\ell} d(R) \leq \operatorname{dist}(Q, R)<2^{\ell+1} d(R)\right\},
$$

$\ell \geq 0$. We want to control the norms of the corresponding pieces $\mathcal{N}_{5, \ell}$ of $\mathcal{N}_{5}$, and this is the only place in this revised Section 9 where it will be more pleasant to use Schur's lemma.

Lemma 9.29 (Schur). Let $\mathcal{N}=((N(Q, R)))_{Q \in \Delta, R \in \Delta}$ be a matrix with complex coefficients, and assume that there are positive numbers $\omega(Q)$, $Q \in \Delta$, such that

$$
\sum_{Q \in \Delta} \frac{\omega(Q)}{\omega(R)}|N(Q, R)| \leq C, \quad \text { for all } R \in \Delta
$$

and

$$
\sum_{R \in \Delta} \frac{\omega(R)}{\omega(Q)}|N(Q, R)| \leq C, \quad \text { for all } Q \in \Delta
$$

Then $\mathcal{N}$ defines a bounded operator on $L^{2}(\Delta)$, with norm $\|\mid \mathcal{N}\| \| \leq C$.

For the very easy proof, see for instance [Da, p. 43] or [My, p. 269]. We want to apply this to $\mathcal{N}_{5, \ell}$, with $\omega(Q)=\mu(Q)^{1 / 2}$. Let us first check sums over $Q$. For $R \in \Delta$,

$$
\begin{aligned}
\sum_{Q} \frac{\omega(Q)}{\omega(R)}\left|N_{5, \ell}(Q, R)\right| & \leq C \sum_{Q \in \mathcal{A}_{\ell}(R)} \mu(Q) d(Q) \operatorname{dist}(Q, R)^{-2} \\
& \leq C A^{-k} d(R)\left(2^{\ell} d(R)\right)^{-2} \sum_{Q \in \mathcal{A}_{\ell}(R)} \mu(Q)
\end{aligned}
$$

by (9.27) and definitions. Since all the cubes $Q \in \mathcal{A}_{\ell}(R)$ lie within $C 2^{\ell} d(R)$ of $R$, their total mass is at most $C 2^{\ell} d(R)$ by (3.1), and so

$$
\sum_{Q} \frac{\omega(Q)}{\omega(R)}\left|N_{5, \ell}(Q, R)\right| \leq C A^{-k} 2^{-\ell}
$$


Next we fix $Q$ and sum over $R$. Of course we need only consider those $R$ for which $Q \in \mathcal{A}_{\ell}(R)$, and all these cubes $R$ lie at distance less or equal than $C 2^{\ell} d(R)=C 2^{\ell} A^{k} d(Q)$ from $Q$. Thus

$$
\begin{aligned}
\sum_{R} \frac{\omega(R)}{\omega(Q)} N_{5, \ell}(Q, R) & \leq C \sum_{R} \mu(R) d(Q) \operatorname{dist}(Q, R)^{-2} \\
& \leq C d(Q)\left(A^{k} 2^{\ell} d(Q)\right)^{-2} \sum_{R} \mu(R) \\
& \leq C d(Q)\left(A^{k} 2^{\ell} d(Q)\right)^{-1} \\
& =C A^{-k} 2^{-\ell}
\end{aligned}
$$

Altogether, Schur's lemma yields

$$
\left\|\left|\mathcal{N}_{5}\right|\right\| \leq \sum_{\ell}||\left|\mathcal{N}_{5, \ell} \|\right| \leq C A^{-k}
$$

Case F. The local part of $A_{2}(Q, R)$ and $B_{12}$. Set $N_{6}(Q, R)=A_{2}(Q, R)$ when $k(Q)=k(R)+k$, (7.37) holds, and $\operatorname{dist}(Q, R)<d(R)$; set $N_{6}(Q, R)=B_{12}$ when $k(Q)=k(R)+k, Q \subset R$ and $Q \neq R$; finally set $N_{6}(Q, R)=0$ otherwise. Note that

$$
N_{6}(Q, R)=\mu(Q)^{1 / 2} \sum_{\substack{R^{*} \in F(R) \\ Q \cap R^{*}=\varnothing}} \mu\left(R^{*}\right)^{-1 / 2} J\left(Q, R^{*} \backslash 2 Q\right),
$$

when $N_{6}(Q, R) \neq 0$, by (7.14) or (7.28). Also, $\operatorname{dist~}(Q, R) \leq d(R)$ when $N_{6}(Q, R) \neq 0$, so for each $Q \in \Delta$ there are at most $C$ cubes $R \in \Delta$ such that $N_{6}(Q, R) \neq 0$. Lemma 9.1 tells us that

$$
\left\|\left|\mathcal{N}_{6}\right|\right\|^{2} \leq C \sup _{R \in \Delta} \Sigma(R)
$$

where $\mathcal{N}_{6}$ is the matrix with coefficients $N_{6}(Q, R)$ and

$$
\Sigma(R)=\sum_{Q} N_{6}(Q, R)^{2}
$$

For each $R \in \Delta$ and $R^{*} \in F(R)$, set

(9.38) $\mathcal{A}\left(R^{*}\right)=\left\{Q \in \Delta_{k(R)+k}\right.$ : $\operatorname{dist}(Q, R) \leq d(R)$ but $\left.Q \cap R^{*}=\varnothing\right\}$. 
Then

$$
\Sigma(R) \leq C \sum_{R^{*} \in F(R)} \mu\left(R^{*}\right)^{-1} \sigma\left(R^{*}\right)
$$

with

$$
\sigma\left(R^{*}\right)=\sum_{Q \in \mathcal{A}\left(R^{*}\right)} \mu(Q) J\left(Q, R^{*} \backslash 2 Q\right)^{2}
$$

Fix $R$ and $R^{*} \in F(R)$. For each $Q \in \mathcal{A}\left(R^{*}\right)$ set

$$
\delta(Q)=d(Q)+\operatorname{dist}\left(Q, R^{*}\right)
$$

and, for notational convenience,

$$
J_{Q}=J\left(Q, R^{*} \backslash 2 Q\right) .
$$

Our basic estimate for $J_{Q}$ is

$$
\begin{aligned}
J_{Q} & =\int_{R^{*} \backslash 2 Q} \frac{d(Q) d \mu(x)}{|x-x(Q)|^{2}} \\
& \leq d(Q) \int_{|x-x(Q)| \geq \delta(Q) / 2} \frac{d \mu(x)}{|x-x(Q)|^{2}} \\
& \leq C \frac{d(Q)}{\delta(Q)}
\end{aligned}
$$

which follows from $(7.10)$, the fact that

$$
\operatorname{dist}\left(x(Q), R^{*}\right) \geq \operatorname{dist}(x(Q), E \backslash Q) \geq d(Q)
$$

(by (3.4) and (3.5)), and (8.1).

Let us first say rapidly how we would estimate $\sigma\left(R^{*}\right)$ if $R^{*}$ were a good cube. We would first sum over the cubes $Q$ such that $\delta(Q) \sim \delta$ for a given $\delta$, the interesting case being when $d(Q) \leq \delta<d\left(R^{*}\right)$. By (9.43), the contribution of $Q$ to the sum would be at most $C \mu(Q)(d(Q) / \delta(Q))^{2}$. Also, the total mass of the cubes $Q$ would be about

$$
\left(\frac{\delta}{d\left(R^{*}\right)}\right)^{\tau} \xi\left(R^{*}\right) \leq C\left(\frac{\delta}{d(R)}\right)^{\tau} \mu\left(R^{*}\right)
$$


(if $R^{*}$ is good) because each cube $Q$ lies at distance less than $C \delta$ from $R^{*}$ but does not meet $R^{*}$. Summing over $Q$ would give less than

$$
C\left(\frac{\delta}{d(R)}\right)^{\tau}\left(\frac{A^{-k} d(R)}{\delta}\right)^{2} \mu\left(R^{*}\right)
$$

We would then sum over $\delta$ and get that

$$
\sigma\left(R^{*}\right) \leq C A^{-k \tau} \mu\left(R^{*}\right)
$$

(the largest terms are when $\delta \sim A^{-k} d(R)$ ).

In general, $R^{*}$ is not a good cube and we'll have to localize to maximal good subcubes of $R^{*}$ and distinguish two cases as usual. For each $Q \in \mathcal{A}\left(R^{*}\right)$, choose a point $z(Q)$ such that

$$
z(Q) \in R^{*} \quad \text { and } \quad \operatorname{dist}(z(Q), Q) \leq \delta(Q) .
$$

Denote by $\mathcal{A}^{+}\left(R^{*}\right)$ the set of cubes $Q \in \mathcal{A}\left(R^{*}\right)$ such that

$$
\begin{aligned}
& z(Q) \text { is contained in a maximal good } \\
& \text { cube } S_{Q} \in S\left(R^{*}\right) \text { and } Q \subset 2 S_{Q} .
\end{aligned}
$$

Also set $\mathcal{A}^{-}\left(R^{*}\right)=\mathcal{A}\left(R^{*}\right) \backslash \mathcal{A}^{+}\left(R^{*}\right)$ and

$$
\sigma_{ \pm}\left(R^{*}\right)=\sum_{Q \in \mathcal{A}^{ \pm}\left(R^{*}\right)} \mu(Q) J_{Q}^{2}
$$

Let us first estimate $\sigma_{+}\left(R^{*}\right)$. A trivial estimate for $J_{Q}$ is

$$
\begin{aligned}
J_{Q} & =\int_{R^{*} \backslash 2 Q} \frac{d(Q) d \mu(x)}{|x-x(Q)|^{2}} \\
& \leq \mu\left(R^{*}\right) d(Q) \operatorname{dist}\left(R^{*}, x(Q)\right)^{-2} \\
& \leq \mu\left(R^{*}\right) d(Q)^{-1}
\end{aligned}
$$

We want to use the following weighted average of (9.47) and (9.43)

$$
J_{Q}^{2} \leq C\left(\frac{d(Q)}{\delta(Q)}\right)^{2-\tau / 2}\left(\frac{\mu\left(R^{*}\right)}{d(Q)}\right)^{\tau / 2} \leq C \frac{d(Q)}{\delta(Q)}\left(\frac{\mu\left(R^{*}\right)}{d(Q)}\right)^{\tau / 2}
$$


For each $S \in S\left(R^{*}\right)$ and $\ell \geq 0$, denote by $B_{\ell}(S)$ the set of cubes $Q \in \mathcal{A}^{+}\left(R^{*}\right)$ such that $2^{\ell} d(Q) \leq \delta(Q)<2^{\ell+1} d(Q)$ and $S=S_{Q}$. Obviously every $Q \in \mathcal{A}^{+}\left(R^{*}\right)$ lies in some $B_{\ell}(S)$ and so

$$
\sigma_{+}\left(R^{*}\right) \leq \sum_{S \in S\left(R^{*}\right)} \sum_{\ell \geq 0} \sum_{Q \in B_{\ell}(S)} \mu(Q) J_{Q}^{2} .
$$

If $Q \in B_{\ell}(S)$, then $Q$ does not meet $S$ (by definition (9.38) of $\mathcal{A}\left(R^{*}\right)$ ) but

$$
\operatorname{dist}(Q, S) \leq \operatorname{dist}(Q, z(Q)) \leq \delta(Q) \leq 2^{\ell+1} d(Q),
$$

by definitions (see in particular (9.44) and (9.45)). Thus $Q \subset N_{t}(S)$, with

$$
t=C 2^{\ell+1} d(Q) d(S)^{-1}=C 2^{\ell+1} A^{-k} d(R) d(S)^{-1} .
$$

Note that $\ell$ cannot be too large: if $B_{\ell}(S)$ contains some $Q$, then $2^{\ell} d(Q) \leq \delta(Q) \leq C d(S)$ because $Q \subset 2 S$ (by (9.45)). In particular, the value of $t$ above is never more than some constant $C$. Set $t^{\prime}=\min \{t, 1\}$. Then all cubes $Q \in B_{\ell}(S)$ still lie in $N_{t^{\prime}}(S)$ (because $Q \subset 2 S$ for $\left.Q \in B_{\ell}(S)\right)$. We may now apply (3.8) and get that

$$
\begin{aligned}
\sum_{Q \in B_{\ell}(S)} \mu(Q) & \leq \mu\left(N_{t^{\prime}}(S)\right) \\
& \leq C\left(2^{\ell} A^{-k} d(R) d(S)^{-1}\right)^{\tau} \xi(S) \\
& \leq C\left(2^{\ell} A^{-k} d(R) d(S)^{-1}\right)^{\tau} \mu(S),
\end{aligned}
$$

because $S$ is a good cube. Next

$$
\begin{aligned}
& \sum_{Q \in B_{\ell}(S)} \mu(Q) J_{Q}^{2} \\
& \quad \leq C\left(2^{\ell} A^{-k} d(R) d(S)^{-1}\right)^{\tau} \mu(S) 2^{-\ell}\left(\frac{\mu\left(R^{*}\right)}{A^{-k} d(R)}\right)^{\tau / 2}
\end{aligned}
$$

by $(9.50),(9.48)$, and the definition of $B_{\ell}(S)$. We may now sum over $\ell \geq 0$, noticing that the largest term is for $\ell=0$, and get less than

$$
C A^{-k \tau / 2}\left(\frac{d(R)}{d(S)}\right)^{\tau}\left(\frac{\mu\left(R^{*}\right)}{d(R)}\right)^{\tau / 2} \mu(S) .
$$

Thus (9.49) becomes

$$
\sigma_{+}\left(R^{*}\right) \leq C A^{-k \tau / 2}\left(\frac{\mu\left(R^{*}\right)}{d(R)}\right)^{\tau / 2} \sum_{S \in S\left(R^{*}\right)}\left(\frac{d(R)}{d(S)}\right)^{\tau} \mu(S) .
$$


By Hölder,

$$
\begin{aligned}
\sum_{S \in S\left(R^{*}\right)}\left(\frac{d(R)}{d(S)}\right)^{\tau} \mu(S) & \leq\left(\sum_{S} \mu(S)\right)^{1-\tau / 2}\left(\sum_{S}\left(\frac{d(R)}{d(S)}\right)^{2} \mu(S)\right)^{\tau / 2} \\
& \leq C \mu\left(R^{*}\right)^{1-\tau / 2} \xi\left(R^{*}\right)^{\tau / 2} \\
& \leq C \mu\left(R^{*}\right)^{1-\tau / 2} d(R)^{\tau / 2}
\end{aligned}
$$

because the cubes $S, S \in S\left(R^{*}\right)$, are disjoint and contained in $R^{*}$, and by Lemma 8.14 and (3.9). Hence

$$
\sigma_{+}\left(R^{*}\right) \leq C A^{-k \tau / 2} \mu\left(R^{*}\right)
$$

which will be enough for our purposes. Let us now turn to $\sigma_{-}\left(R^{*}\right)$. First we want to check that

$$
J_{Q} \leq C A^{-k}, \quad \text { for all } Q \in \mathcal{A}^{-}\left(R^{+}\right) .
$$

We start with the easy case when $Q$ is not contained in $2 R^{*}$. If $d(Q) \geq$ $d\left(R^{*}\right)$, then

$$
\operatorname{dist}\left(x(Q), R^{*}\right) \geq \operatorname{dist}(x(Q), E \backslash Q) \geq d(Q) \geq d\left(R^{*}\right),
$$

by definition (9.38) of $\mathcal{A}\left(R^{*}\right)$, (3.4) and (3.5). Otherwise, $\operatorname{diam} Q<$ $d\left(R^{*}\right) / 2$ and, since some point of $Q$ lies at distance $>d\left(R^{*}\right)$ from $R^{*}$, $\operatorname{dist}\left(x(Q), R^{*}\right) \geq d\left(R^{*}\right) / 2$. In both cases

$$
\begin{aligned}
J_{Q} & \leq \mu\left(R^{*}\right) d(Q) \operatorname{dist}\left(x(Q), R^{*}\right)^{-2} \\
& \leq 4 \mu\left(R^{*}\right) d(Q) d\left(R^{*}\right)^{-2} \\
& \leq C A^{-k} \mu\left(R^{*}\right) d(R)^{-1} \\
& \leq C A^{-k}
\end{aligned}
$$

by $(9.42),(7.10)$ and (3.9).

We still need to check (9.55) when $Q \subset 2 R^{*}$. Let $H_{0}=R^{*} \supset$ $H_{1} \supset \cdots \supset H_{\ell}$ be the decreasing sequence of all cubes $H \subset R^{*}$ that contain $z(Q)$ (the point of $R^{*}$ that was chosen in (9.44)) and such that $Q \subset 2 H$. Since $Q \subset 2 R^{*}$, there is at least one such cube, and then $d\left(H_{\ell}\right) \leq C \delta(Q)$ by minimality of $H_{\ell}$ (and (9.41)). Note also that all 
the cubes $H_{j}, 0 \leq j \leq \ell$, are bad because $Q \in \mathcal{A}^{-}\left(R^{*}\right)$ and (9.45) does not hold. Thus (3.9) and (3.12) yield

$$
\mu\left(2 H_{j}\right) \leq C \xi\left(H_{j}\right) \leq C A^{-10 j} \xi\left(R^{*}\right) \leq C A^{-10 j} d\left(R^{*}\right) .
$$

Decompose $R^{*} \backslash 2 Q$ into the sets $Z_{j}=\left(R^{*} \backslash 2 Q\right) \cap\left(2 H_{j} \backslash 2 H_{j+1}\right), 0 \leq$ $j \leq \ell-1$, and $Z_{\ell}=\left(R^{*} \backslash 2 Q\right) \cap 2 H_{\ell}$. When $0 \leq j \leq \ell-2$ and $x \in Z_{j}$,

$$
\begin{aligned}
|x-x(Q)| & \geq \operatorname{dist}(x, Q) \\
& \geq \operatorname{dist}\left(x, 2 H_{\ell}\right) \\
& \geq \operatorname{dist}\left(E \backslash 2 H_{j+1}, 2 H_{\ell}\right) \\
& \geq \frac{1}{2} d\left(H_{j+1}\right) .
\end{aligned}
$$

Thus, for $0 \leq j \leq \ell-2$,

$$
J\left(Q, Z_{j}\right) \leq 4 \mu\left(Z_{j}\right) d(Q) d\left(H_{j+1}\right)^{-2} \leq C A^{-8 j} A^{-k},
$$

by (7.10) and (9.56).

When $j=\ell-1$ or $j=\ell$, we want to use the simple estimate

$$
|x-x(Q)| \geq \frac{\delta(Q)}{2}, \quad \text { for } x \in R^{*}
$$

which comes from the fact that $|x-x(Q)| \geq \operatorname{dist}\left(Q, R^{*}\right)$ trivially and $|x-x(Q)| \geq \operatorname{dist}(x(Q), E \backslash Q) \geq d(Q)$ by (9.38), (3.4) and (3.5). (See also the definition (9.41).) Thus, for $j=\ell-1$ and $j=\ell$,

$$
J\left(Q, Z_{j}\right) \leq 4 \mu\left(Z_{j}\right) d(Q) \delta(Q)^{-2} \leq C A^{-10 \ell} A^{-k} d(R)^{2} \delta(Q)^{-2}
$$

Recall that $d\left(H_{\ell}\right) \leq C \delta(Q)$, so that

$$
A^{-10 \ell}=\left(\frac{d\left(H_{\ell}\right)}{d\left(R^{*}\right)}\right)^{10} \leq C\left(\frac{\delta(Q)}{d(R)}\right)^{10} .
$$

Since we also have that $\delta(Q) \leq C d\left(R^{*}\right)$ because $Q \subset 2 R^{*},(9.60)$ implies that

$$
J\left(Q, Z_{j}\right) \leq C A^{-k}\left(\frac{\delta(Q)}{d(R)}\right)^{8} \leq C A^{-k},
$$


when $j=\ell-1$ or $j=\ell$. Altogether

$$
J_{Q}=J\left(Q, R^{*} \backslash 2 Q\right) \leq \sum_{j=0}^{\ell} J\left(Q, Z_{j}\right) \leq C A^{-k},
$$

which completes our proof of (9.55).

A second estimate for $J_{Q}$ is

$$
J_{Q} \leq \mu\left(R^{*}\right) d(Q) \operatorname{dist}\left(x(Q), R^{*}\right)^{-2} \leq 4 \mu\left(R^{*}\right) d(Q) \delta(Q)^{-2},
$$

which follows directly from the definitions (9.42) and (7.10), and (9.59).

Plug these two estimates into (9.46) to get

$$
\sigma_{-}\left(R^{*}\right) \leq C A^{-k} \sum_{Q \in \mathcal{A}^{-}\left(R^{*}\right)} \mu(Q) \mu\left(R^{*}\right) d(Q) \delta(Q)^{-2}
$$

When we sum over the set of cubes $Q$ such that $\delta(Q) \geq A^{-k / 2} d(R)$, we get less than

$$
\begin{aligned}
C A^{-k}\left(\sum_{Q} \mu(Q)\right) \mu & \left(R^{*}\right) A^{-k} d(R) A^{k} d(R)^{-2} \\
& \leq C A^{-k} \mu\left(R^{*}\right)\left(\sum_{Q} \mu(Q)\right) d(R)^{-1} \\
& \leq C A^{-k} \mu\left(R^{*}\right),
\end{aligned}
$$

by $(3.1)$ or $(3.9)$.

We are left with the cubes $Q$ such that $\delta(Q) \leq A^{-k / 2} d(R)$. These cubes are contained in $N_{t}\left(R^{*}\right)$, with $t=\min \left\{1, C A^{-k / 2}\right\}$ because they are $\delta(Q)$-close to $R^{*}$ but do not meet it (by (9.38)). By (3.8) and (3.9), their total mass is at most

$$
C A^{-k \tau / 2} \xi\left(R^{*}\right) \leq C A^{-k \tau / 2} d(R),
$$

and so the corresponding piece of $\sigma_{-}\left(R^{*}\right)$ is at most

$$
C A^{-k} A^{-k \tau / 2} d(R) \mu\left(R^{*}\right)\left(A^{-k} d(R)\right)^{-1} \leq C A^{-k \tau / 2} \mu\left(R^{*}\right) .
$$

Altogether, $\sigma_{-}\left(R^{*}\right) \leq C A^{-k \tau / 2} \mu\left(R^{*}\right)$. Now

$$
\sigma\left(R^{*}\right)=\sigma_{+}\left(R^{*}\right)+\sigma_{-}\left(R^{*}\right) \leq C A^{-k \tau / 2} \mu\left(R^{*}\right),
$$


by (9.40), (9.46), (9.54) and this last estimate. We may now compare with (9.39) and (9.37) to get that $\left\|\left|\mathcal{N}_{6}\right|\right\| \leq C A^{-k \tau / 4}$, as desired.

Case G. The terms $B_{22}$. Finally define $\mathcal{N}_{7}$ by taking $N_{7}(Q, R)=B_{22}$ when $Q \subset R, Q \neq R$, and $k(Q)=k(R)+k$, and $N_{7}(Q, R)=0$ otherwise. This is the last piece of the matrix $\mathcal{N}^{k}$ that we have to study: recall that $\mathcal{N}$ was defined around (7.36)-(7.39), and that coefficients $A_{1}(Q, R)$ and $A_{2}(Q, R)$ were dealt with in subsections $\mathrm{B}, \mathrm{E}$ and $\mathrm{F}$ respectively, while $A_{3}(Q, R)$ was treated in Subsection $\mathrm{A}, B_{11}$ in Subsection $\mathrm{C}, B_{12}$ in $\mathrm{F}$ and $B_{21}$ in D.

Recall from (7.30) that

$$
B_{22}=\mu(Q)^{1 / 2} \mu(R(Q))^{-1 / 2} J(Q, E \backslash(2 Q \cup R(Q))),
$$

where $R(Q)$ is the child of $R$ that contains $Q$. As usual we can apply Lemma 9.1, and

$$
\left\|\mid \mathcal{N}_{7}\right\| \|^{2} \leq \sup _{R \in \Delta} \Sigma(R)
$$

with

$$
\Sigma(R)=\sum_{R^{*} \in F(R)} \sum_{Q \in \mathcal{A}\left(R^{*}\right)} \mu(Q) \mu\left(R^{*}\right)^{-1} J_{Q}^{2},
$$

where this time we set

$$
\mathcal{A}\left(R^{*}\right)=\left\{Q \in \Delta_{k(R)+k}: Q \subset R^{*}\right\}
$$

and

$$
J_{Q}=J\left(Q, E \backslash\left(2 Q \cup R^{*}\right)\right) .
$$

Set $\delta(Q)=d(Q)+\operatorname{dist}\left(Q, E \backslash R^{*}\right)$ for $Q \in \mathcal{A}\left(R^{*}\right)$. Note that

$$
|x-x(Q)| \geq \frac{\delta(Q)}{2}, \quad \text { for } x \in E \backslash\left(2 Q \cup R^{*}\right),
$$

because $|x-x(Q)| \geq d(Q)$ on $E \backslash 2 Q$ and $|x-x(Q)| \geq \operatorname{dist}\left(Q, E \backslash R^{*}\right)$ on $E \backslash R^{*}$. Then

$$
J_{Q}=d(Q) \int_{E \backslash\left(2 Q \cup R^{*}\right)} \frac{d \mu(x)}{|x-x(Q)|^{2}} \leq C d(Q) \delta(Q)^{-1},
$$


by (8.1).

When $k \leq 10$, say, we can simply say that $J_{Q} \leq C$ by $(9.71)$ and $\Sigma(R) \leq C$ by summing brutally. When $k \geq 10$, we expect to win something from (9.71) when $\delta(Q) \gg d(Q)$, and otherwise to use the fact that $Q$ stays close to the "boundary of $R^{*}$ " to say that $\sum \mu(Q)$ is small. As usual we need to distinguish cases because $R^{*}$ is not necessarily good.

Fix $R^{*} \in F(R)$ and first consider

$$
\begin{aligned}
& \mathcal{A}^{+}=\left\{Q \in \mathcal{A}\left(R^{*}\right):\right. \text { there is a maximal good cube } \\
&\left.S \in S\left(R^{*}\right) \text { such that } k(S) \leq k(R)+\frac{k}{2} \text { and } Q \subset S\right\} .
\end{aligned}
$$

For each $S \in S\left(R^{*}\right)$ and $\ell \geq 0$, set

$$
\mathcal{A}_{\ell}^{+}(S)=\left\{Q \in \mathcal{A}^{+}: Q \subset S \text { and } 2^{\ell} d(Q) \leq \delta(Q)<2^{\ell+1} d(Q)\right\}
$$

All these cubes lie at distance less than $2^{\ell+1} d(Q)$ from $E \backslash R^{*}$, and so they lie in $N_{t}(S)$, with $t=C 2^{\ell} d(Q) d(S)^{-1}$. If we get a $t \geq 1$, simply remember that $Q \in \mathcal{A}_{\ell}^{+}(S)$ is always contained in $S$; otherwise apply (3.8) and the fact that $S$ is a good cube to get that

$$
\sum_{Q \in \mathcal{A}_{\ell}^{+}(S)} \mu(Q) J_{Q}^{2} \leq C\left(2^{\ell} A^{-k} d(R) d(S)^{-1}\right)^{\tau} \mu(S) 2^{-2 \ell}
$$

where the $2^{-2 \ell}$ comes from (9.71). When we sum this over $\ell \geq 0$, the largest term is when $\ell=0$ and we get at most

$$
C A^{-k \tau} d(R)^{\tau} d(S)^{-\tau} \mu(S) \leq C A^{-k \tau / 2} \mu(S),
$$

because only the maximal good cubes $S$ with $k(S) \leq k(R)+k / 2$ can give non empty sets $\mathcal{A}_{\ell}^{+}(S)$, by (9.72). Since every cube $Q \in \mathcal{A}^{+}$lies in some $\mathcal{A}_{\ell}^{+}(S)$,

$$
\begin{aligned}
\sum_{Q \in \mathcal{A}^{+}} \mu(Q) \mu\left(R^{*}\right)^{-1} J_{Q}^{2} & \leq C A^{-k \tau / 2} \mu\left(R^{*}\right)^{-1} \sum_{S \in S\left(R^{*}\right)} \mu(S) \\
& \leq C A^{-k \tau / 2}
\end{aligned}
$$

Next we want to estimate the contribution of $\mathcal{A}^{-}=\mathcal{A}\left(R^{*}\right) \backslash \mathcal{A}^{+}$to $\sum(R)$ (in (9.67)). Let $Q \in \mathcal{A}^{-}$be given, and let $H_{0}=R^{*} \supset H_{1} \supset \cdots \supset H_{\ell}$ be the collection of all subcubes of $R^{*}$ that contain $Q$ and are of generation 
less or equal than $k(R)+k / 2$. By the definition (9.72) of $\mathcal{A}^{+}$, all these cubes are bad, and so

$$
\mu\left(2 H_{j}\right) \leq C \xi\left(H_{j}\right) \leq C A^{-10 j} \xi\left(R^{*}\right) \leq C A^{-10 j} d(R),
$$

by (3.9), (3.12), and (3.9) again. Now

$$
J_{Q}=J\left(Q, E \backslash\left(2 Q \cup R^{*}\right)\right) \leq \sum_{j=0}^{\ell+1} J\left(Q, Z_{j}\right),
$$

where $Z_{0}=E \backslash 2 R^{*}, Z_{j}=2 H_{j-1} \backslash 2 H_{j}$ for $1 \leq j \leq \ell$, and $Z_{\ell+1}=$ $2 H_{\ell} \backslash 2 Q$. This comes directly from the definitions (9.69) and (7.10). On $Z_{j}, 0 \leq j \leq \ell$,

$$
|x-x(Q)| \geq \operatorname{dist}\left(E \backslash 2 H_{j}, Q\right) \geq d\left(H_{j}\right)=A^{-j} d\left(R^{*}\right),
$$

because $Q \subset H_{j}$. Thus, for $1 \leq j \leq \ell$,

$$
J\left(Q, Z_{j}\right) \leq d(Q) A^{2 j} d\left(R^{*}\right)^{-2} \mu\left(Z_{j}\right) \leq C A^{-8 j} A^{-k},
$$

by $(9.76)$. For $j=0$, we simply have that

$$
\begin{aligned}
J\left(Q, Z_{0}\right) & =d(Q) \int_{E \backslash 2 R^{*}} \frac{d \mu(x)}{|x-x(Q)|^{2}} \\
& \leq C d(Q) d\left(R^{*}\right)^{-1} \\
& \leq C A^{-k},
\end{aligned}
$$

because dist $\left(x(Q), E \backslash 2 R^{*}\right) \geq \operatorname{dist}\left(Q, E \backslash 2 R^{*}\right) \geq d\left(R^{*}\right)$ (since $Q \subset R^{*}$ ), and by (8.1). Finally, $|x-x(Q)| \geq d(Q)$ on $Z_{\ell+1}$ and so

$$
J\left(Q, Z_{\ell+1}\right) \leq d(Q)^{-1} \mu\left(2 H_{\ell}\right) \leq C A^{k} A^{-10 \ell} \leq C A^{-k},
$$

because $H_{\ell}$ is the smallest cube $H$ containing $Q$ and for which $k(H) \leq$ $k(R)+k / 2$. Summing over $\ell$ now gives that $J_{Q} \leq C A^{-k}$ for all $Q \in \mathcal{A}^{-}$, and then

$$
\sum_{Q \in \mathcal{A}^{-}} \mu(Q) \mu\left(R^{*}\right)^{-1} J_{Q}^{2} \leq C A^{-2 k}
$$

because all these cubes are disjoint and contained in $R^{*}$. Finally, when we add up the estimates in (9.75) and (9.81) and then sum over $R^{*} \in$ 
$F(R)$, we get that $\Sigma(R) \leq C A^{-k \tau / 2}$ and $\left\|\left|\mathcal{N}_{7}\right|\right\| \leq C A^{-k \tau / 4}$ (see (9.67) and (9.66)).

At this point we may collect all the estimates from the various subsections. We get that

$$
\left|\left\|\mathcal { N } ^ { k } \left|\left\|\left|\leq \sum_{j}\right||| \mathcal{N}_{j} \mid\right\| \leq C A^{-k \tau / 4}\right.\right.\right.
$$

and finally

$$
\||\mathcal{N}|\| \leq \sum_{k}||\left|\mathcal{N}^{k}\right| \| \leq C
$$

This completes the proof of Theorem 3.20.

REMARK 9.82. We have only used the fact that the ambient dimension is 2 a few times, when we used (8.1) to estimate the number of cubes $Q \in \Delta_{k(R)+k}$ in a ball of radius $C d(R)$. This estimate was always beaten by a $A^{-10 k}$ that came from (3.12). If we had been working in a larger ambient dimension, we would only have needed to replace 10 with a larger constant in (3.12), which is possible. Thus Theorem 3.20 works also for one-dimensional sets $E \subset R^{n}$, with almost the same proof. The proof most probably also works for different dimensions of $E$ (and corresponding homogeneities of kernel estimates) but we did not check this carefully. The authors of [NTV] did for their version.

\section{A short description of $[\mathrm{DM}]$.}

We want to use Theorem 3.20 to prove our theorem about analytic capacity. So we give ourselves a compact set $E \subset \mathbb{C}$ such that $H^{1}(E)<$ $+\infty$ and $E$ has positive analytic capacity, and we want to show that $E$ is not totally unrectifiable. As we discussed in the introduction, we can find a bounded measurable function $f$ on $E$ such that $\int f d \mu=a>0$ and the Cauchy integral of $f d \mu$ is bounded on $\mathbb{C} \backslash E$. Here $\mu$ denotes the restriction of $H^{1}$ to $E$.

Next we want to replace $f d \mu$ with a new measure $g d \nu$, where $g$ has the advantage of being accretive (i.e., satisfies (2.6)). We shall use the measure $\nu$ and the function $g$ constructed in $[\mathrm{DM}]$ for purposes similar to those of this paper. These satisfy (1.5)-(1.8), and also a weaker analogue of (1.9), namely, the fact that the maximal Cauchy integral of $g d \nu$ lies in $L^{2}(d \nu)$. To complete the argument outlined in the introduction, we shall have to put ourselves in position to apply Theorem 3.20 
to the measure $\nu$, and in particular construct an acceptable collection of dyadic cubes on the support of $\nu$. These cubes will be constructed as modifications of the dyadic cubes on $E$ given by [DM]; see the next section. Once this is done and we are in positition to apply Theorem 3.20 we shall have also to check that truncated Cauchy integrals of $g d \nu$ lie in the relevant BMO-space (instead of just $L^{2}$ ) uniformly. This will only be possible after we give a reasonable description of the construction of $g$ and $\nu$, which is the aim of this section. It will be convenient to use references like $(* 1.2)$ rather than the longer "[DM, (1.2)]".

We start with our compact set $E \subset \mathbb{C}, d \mu=d H_{\mid E}^{1}$, and a bounded function $f$ such that $\|f\|_{\infty} \leq 1$ and $\int f d \mu=a>0$. The construction of $\nu$ and $g$ will only use these informations; it will happen that in addition the Cauchy integral of $f d \mu$ is bounded on $\mathbb{C} \backslash E$, and then $g d \mu$ will also have nice properties with respect to the Cauchy kernel, but we don't need to think about this now.

The first thing we do is construct a collection $\Delta=\bigcup_{k \geq 0} \Delta_{k}$ of dyadic cubes with the properties listed below. Note that $\mu$ is a finite measure, but does not necessarily satisfy (3.1); this will not be a problem. The constants $C_{1}, C_{2}, A$, below are absolute constants; see the discussion below. Let us describe the properties of $\Delta$. First

For each $k \geq 0, E$ is the disjoint union of the Borel sets $Q, Q \in \Delta_{k}$,

$$
\begin{aligned}
& \text { if } k<\ell, Q \in \Delta_{k} \text { and } R \in \Delta_{\ell}, \\
& \text { then } Q \cap R=\varnothing \text { or else } R \subset Q,
\end{aligned}
$$

and for each $k \geq 0$ and each cube $Q \in \Delta_{k}$, there is a ball $B(Q)=$ $B(x(Q), r(Q))$, centered on $E$, and such that

$$
\begin{gathered}
A^{-k} \leq r(Q) \leq C_{1} A^{-k}, \\
E \cap B(Q) \subset Q \subset E \cap 28 B(Q),
\end{gathered}
$$

and

$$
\text { the balls } 5 B(Q), Q \in \Delta_{k} \text {, are disjoint. }
$$

These are the properties $(* 3.3)-(* 3.9)$ in Theorem $* 3.2$. It is also easy to arrange that

$\Delta_{0}$ has only one element. 
This was assumed in $[\mathrm{DM}]$ also (see just after $(* 4.1)$; the construction gives this automatically if we normalize things by taking $\operatorname{diam} E=1$. Next there is the story about small boundaries. Set

$$
\begin{aligned}
N_{t}(Q)= & \left\{x \in E \backslash Q: \operatorname{dist}(x, Q) \leq t A^{-k(Q)}\right\} \\
& \cup\left\{x \in Q: \operatorname{dist}(x, E \backslash Q) \leq t A^{-k(Q)}\right\},
\end{aligned}
$$

for $Q \in \Delta$ and $0<t \leq 1$, and where $k(Q)$ denotes, as always, the generation of $Q$. Then

$$
\mu\left(N_{t}(Q)\right) \leq C_{2} t^{\tau} \mu(90 B(Q))
$$

for all $Q \in \Delta$ and $0<t \leq 1$, and where we can take the constant $\tau>1$ as close to 1 as we want. Here we shall take $\tau=9 / 10$. Furthermore we can decompose $\Delta$ into the set of good cubes $Q$ such that

$$
\mu\left(10^{4} B(Q)\right) \leq C_{1} \mu(Q),
$$

and the set of bad cubes that do not satisfy (10.9) but for which

$$
r(Q)=A^{-k(Q)}
$$

and, more importantly,

$$
\mu\left(10^{4} B(Q)\right) \leq A^{-10} \mu\left(10^{4} B(\hat{Q})\right),
$$

where $\hat{Q}$ denotes the parent of $Q$. Note that the only cube of $\Delta_{0}$ is good by definitions, and so $\hat{Q}$ is defined for all bad cubes.

These are not exactly the condition $(* 3.13)-(* 3.16)$ stated in Theorem $* 3.2$. First, there is the difference that we replaced $100 B(Q)$ in $(* 3.16)$ with $10^{4} B(Q)$. This does not cause any harm; it just makes some of the constants larger. The second difference is in the phrasing of the conditions: $(10.8)-(10.11)$ are are slightly different from $(* 3.13)$ $(* 3.16)$, even with $10^{4}$ instead of 100 , but they are fairly easy to deduce from $(* 3.13)-(* 3.16)$ by choosing $C_{1}$ and $A$ large enough. In fact, this is what was done in $[\mathrm{DM}]$, in sections 4 and 5 . Theorem $* 3.2$ was stated for all choices of $C_{1}$ (which is called $C_{0}$ there) and $A$, provided that $C_{1}>1$ and $A>5000 C_{1}$, but then it was decided to take $A=C C_{1}^{100}$ for some absolute constant $C$ (the one that shows up in $(* 3.13))$ and then $C_{1}$ so large that $(* 3.13)$ and $(* 3.16)$ actually imply $(10.8)$ and 
(10.11). See $(* 4.1)$ for the choice of $A,(* 5.25)$ and $(* 5.26)$ for a discretized version of (10.8) where $t=A^{-\ell}=\left(C C_{1}^{100}\right)^{-\ell}$ and we get $\mu\left(N_{t}(Q)\right) \leq C_{1}^{-93 \ell} \mu(90 B(Q)) \leq C^{\prime} t^{9 / 10} \mu(90 B(Q))$, and $(* 5.30)$ for (10.11). The two other relations (10.9) and (10.10) are the same as $(* 3.14)$ and $(* 3.15)$.

This completes our discussion of the construction of cubes in [DM]. Note that we get our implicit property that $A \gg C_{1}$ from earlier sections automatically here (i.e., without having to skip generations artificially).

Once our collection of cubes is chosen, we run a stopping time construction, somewhat like in [Ch2]. We select collections $I_{1}$ and $L I$ of cubes $Q \in \Delta$, with the following main properties:

(10.12) the cubes of $I_{1} \cup L I$ are disjoint (this is $(* 4.11)$ ) and,

all the cubes $Q \in \Delta$ such that $Q \subset \mathcal{O}(M)$ or

$\operatorname{Re} \int_{Q} f d \mu \leq a_{1} \mu(Q)$ are contained in some

cube of $I_{1} \cup L I$,

where $\mathcal{O}(M)=\{x \in E$ : there is an $r>0$ such that $\mu(B(x, r))>M r\}$, and $M$ and $a_{1}$ are two positive constants (that may depend wildly on $E)$. This is Remark $* 4.12$; see also $(* 4.4)$ and $(* 4.5)$ for the definition of $\mathcal{O}(M)$. Set

$$
\begin{aligned}
& \Delta^{0}=\left\{Q \in \Delta: Q \in I_{1} \cup L I \text { or } Q\right. \text { is not } \\
&\text { contained in any cube of } \left.I_{1} \cup L I\right\} .
\end{aligned}
$$

These are the cubes which we shall really be working with. A fairly easy consequence of $(10.13)$ (see $(* 4.13)$ ) is that

$$
\mu(100 B(Q)) \leq C A^{-k(Q)}, \quad \text { for all } Q \in \Delta^{0} .
$$

Denote by $P L I$ the set of parents of cubes of $L I$. This makes sense because the only cube of $\Delta_{0}$ happens not to be in $L I$ (or $I_{1}$ either), by construction. Set $I=I_{1} \cup P L I$. One puts a suitable order on $I$; this order is chosen so that cubes of earlier generations come first and, in case of equality, cubes of $I_{1} \cap \Delta_{k}$ come before cubes of $P L I \cap \Delta_{k}$. Call $Q_{n}, n \geq 1$, the $n^{\text {th }}$ cube of $I$ for this order. We construct a sequence of measures $F_{n}, n \geq 0$, as follows.

All measures $F_{n}$ are of the type

$$
F_{n}=\rho_{n} f d \mu+\sum_{1 \leq m \leq n} \alpha_{m} d \nu_{m}
$$


(see $(* 4.15)$ ), where $\left\{\rho_{n}\right\}$ is a decreasing sequence of nonnegative functions on $E$, with $0 \leq \rho_{n} \leq 1$, the $\alpha_{m}$ 's are bounded complex numbers, and $d \nu_{m}$ is a finite sum of multiples of restrictions of Hausdorff measure on circles.

We start with $F_{0}=f d \mu, \rho_{0} \equiv 1$, and no measure $\nu_{0}$, and construct the $F_{n}$ by induction. To go from $F_{n-1}$ to $F_{n}$, we distinguish between two cases. When $Q_{n} \in I_{1}$, we simply replace $Q_{n}$ with a circle, as follows. Take $\rho_{n}=\rho_{n-1} \mathbf{1}_{E \backslash Q_{n}}$ (i.e., kill the part of $\rho_{n-1} f d \mu$ that lives on $Q_{n}$ ) and choose $\mathcal{C}_{n}=\mathcal{C}\left(Q_{n}\right)$, where

(10.17) $\mathcal{C}(Q)$ denotes the circle with center $x(Q)$ and radius $\frac{r(Q)}{100}$,

and $x(Q), r(Q)$ are as in (10.3)-(10.5). In [DM] we chose a slightly larger radius for $\mathcal{C}(Q)$ (see $(* 4.2)$ ), but this new choice does not make any difference in $[\mathrm{DM}]$, and will help us a little bit here. Finally choose

$$
d \nu_{n}=\rho_{n}^{*} \frac{\mu\left(Q_{n}\right)}{H^{1}\left(\mathcal{C}_{n}\right)} d H_{\mid \mathcal{C}_{n}}^{1}
$$

where $\rho_{n}^{*}$ denotes the value of $\rho_{n-1}$ on $Q_{n}$, which happens to be constant by construction. Take $\alpha_{n}=\mu\left(Q_{n}\right)^{-1} \int_{Q_{n}} f d \mu$, so as to get $\int F_{n}=$ $\int F_{n-1}$.

When $Q_{n} \in P L I$, the construction is slightly more complicated. We want to remove the children of $Q_{n}$ that lie in $L I$ and replace them with circles, but we shall also modify the values of $\rho_{n-1} f$ on the rest of $Q$. Denote by $\mathcal{A}_{n}$ the set of children of $Q$ that lie in $L I$ and by $\mathcal{A}_{n}^{*}$ the set of other children of $Q$ (i.e., those that do not lie in $L I$ ). Set $H_{n}=\bigcup_{Q \in \mathcal{A}_{n}} Q, G_{n}=\bigcup_{Q \in \mathcal{A}_{n}^{*}} Q$, and then

$$
\rho_{n}(x)= \begin{cases}\rho_{n-1}(x), & \text { when } x \in E \backslash Q_{n}, \\ 0, & \text { when } x \in H_{n}, \\ \left(1-\theta_{n}\right) \rho_{n-1}(x), & \text { when } x \in G_{n},\end{cases}
$$

where the number $0 \leq \theta_{n}<1$ is correctly chosen (see $(* 4.28)$ and $(* 4.32))$. Also set

$$
\mathcal{C}_{n}=\sum_{Q \in \mathcal{A}_{n}} \mathcal{C}(Q)
$$

and

$$
d \nu_{n}=\sum_{Q \in \mathcal{A}_{n}} \rho_{n}^{*} \frac{\mu(Q)}{H^{1}(\mathcal{C}(Q))} d H_{\mid \mathcal{C}(Q)}^{1}
$$


where $\rho_{n}^{*}$ still denotes the constant value of $\rho_{n-1}$ on $Q_{n}$. This is slightly different from the choice given in $[\mathrm{DM}]$, where $\mathcal{C}_{n}$ was taken to be only one of the $\mathcal{C}(Q), Q \in \mathcal{A}_{n}$, chosen at random, and on which we put the total mass of $H_{n}$. This modification will make our life a little more pleasant later (when we compare the mass repartitions of $\mu$ and $\nu$ ), but it does not alter the argument in [DM]. The main point, of course, is that we still have the same mass

$$
\left\|\nu_{n}\right\|=\rho_{n}^{*} \mu\left(H_{n}\right) \text {. }
$$

To complete the definition of $F_{n}$ when $Q_{n} \in P L I$, one also chooses a complex number $\alpha_{n}$ and sets

$$
F_{n}=F_{n-1}-\mathbf{1}_{H_{n}} \rho_{n-1} f d \mu-\theta_{n} \mathbf{1}_{G_{n}} \rho_{n-1} f d \mu+\alpha_{n} d \nu_{n},
$$

We don't need to be too precise here about the way the constants $\alpha_{n}$ and $\theta_{n}$ were chosen. The main constraint was that

$$
\int F_{n}=\int F_{n-1},
$$

our choices were such that

$$
0 \leq \theta_{n} \leq C \frac{\mu\left(H_{n}\right)}{\mu\left(Q_{n}\right)}
$$

and

$$
\left|\alpha_{n}\right| \leq C
$$

(see $(* 4.33)$ and $(* 4.38)$ ).

It is a good idea to set $\mathcal{A}_{n}=\left\{Q_{n}\right\}, \mathcal{A}_{n}^{*}=\varnothing$ (say, but it does not matter) when $Q_{n} \in I_{1}$. With these conventions, we still have the properties (10.18)-(10.22) when $Q_{n} \in I_{1}$ (see $(* 4.21)-(* 4.23)$ ).

We may also have to use later the fact that

$$
\text { the sets } H_{n}, n \geq 1 \text {, are disjoint, }
$$

which comes from (10.12) and the fact that each $H_{n}$ is the (disjoint) union of the cubes of $\mathcal{A}_{n}$. Alternatively, see $(* 4.69)$ for this statement.

Since $\left\{\rho_{n}\right\}$ is a decreasing sequence of nonnegative functions, it has a limit $\rho_{\infty}$. Set

$$
E_{\infty}=\left\{x \in E: \rho_{\infty}(x)>0\right\}
$$


By construction, $E_{\infty}$ does not meet any cube of $I_{1} \cup L I$. Then

$(10.28) \operatorname{dist}\left(\mathcal{C}(Q), E_{\infty}\right) \geq \operatorname{dist}(\mathcal{C}(Q), E \backslash Q) \geq \frac{99}{100} r(Q) \geq \frac{99}{100} d(Q)$

for $Q \in I_{1} \cup L I$, by (10.17), (10.4), and (10.3).

Similarly, if $Q$ and $Q^{\prime} \in \Delta$ are such that $Q \cap Q^{\prime}=\varnothing$, (10.4) says that $\left|x(Q)-x\left(Q^{\prime}\right)\right| \geq \max \left\{r(Q), r\left(Q^{\prime}\right)\right\}$, and hence

$$
\operatorname{dist}\left(\mathcal{C}(Q), \mathcal{C}\left(Q^{\prime}\right)\right) \geq \frac{98}{100} \max \left\{r(Q), r\left(Q^{\prime}\right)\right\}
$$

This is the case in particular when $Q, Q^{\prime} \in I_{1} \cup L I$ and $Q \neq Q^{\prime}$.

The measure that we want to study is

$$
d \nu=\rho_{\infty} d \mu+\sum_{n} d \nu_{n}
$$

which is obviously finite because $\mu$ is, and by (10.21) and (10.26). The function $g$ is given by

$$
\begin{cases}g(x)=f(x), & \text { on } E_{\infty}, \\ g(x)=\alpha_{n}, & \text { on } \mathcal{C}_{n},\end{cases}
$$

which does not cause any confusion because all these sets are disjoint by (10.28), (10.29), and (10.30).

The function $g$ turns out to be bounded (by (10.25)) and accretive (which means that it satisfies (2.6)) by construction. This comes from the whole design of the stopping time argument (and in particular (10.13)) and the choice of the coefficients $\alpha_{n}$, but we don't need to know precisely how it is proved to understand the rest of the present paper. See $(* 2.6)$ and its proof before Lemma $* 4.56$ for details.

Our next task is to define a collection of cubes $\tilde{\Delta}$ on the support of $\nu$, and then prove a $T(b)$-theorem for $\nu$ and these cubes. This is the aim of the two next sections.

\section{Dyadic cubes for $\nu$ and $\nu^{+}$.}

The following measure $\nu^{+}$will be slightly easier to handle than $\nu$. Set

$$
d \nu^{+}=\mathbf{1}_{E_{\infty}} d \mu+\sum_{n} d \nu_{n}^{+}
$$


where

$$
d \nu_{n}^{+}=\left(\rho_{n}^{*}\right)^{-1} d \nu_{n}=\sum_{Q \in \mathcal{A}_{n}} \frac{\mu(Q)}{H^{1}(\mathcal{C}(Q))} d H_{\mid \mathcal{C}(Q)}^{1}
$$

Obviously $\nu \leq \nu^{+}$, and $\nu^{+}$is still a finite measure because $\mu$ is finite, and by (10.21) and (10.26). Set

$$
\tilde{E}=E_{\infty} \cup\left(\bigcup_{n \geq 1} \mathcal{C}_{n}\right)=E_{\infty} \cup\left(\bigcup_{Q \in I_{1} \cup L I} \mathcal{C}(Q)\right) .
$$

This is not quite the support of $\nu^{+}$, because $\operatorname{supp} \nu^{+}$is closed, but on the other hand

$$
\nu^{+}(\mathbb{C} \backslash \tilde{E})=0
$$

which will be enough for our purposes.

In this section we want to construct families $\tilde{\Delta}_{k}$ of partitions of $\tilde{E}$ and check that they satisfy the conditions (3.1)-(3.12) required for Theorem 3.20, with respect to the measure $\nu^{+}$. Let us start with the construction of cubes.

For each cube $Q \in \Delta^{0}$ (see (10.14) for the definition), set

$$
R(Q)=\left(Q \cap E_{\infty}\right) \cup\left(\bigcup_{\substack{S \in I_{1} \cup L I \\ S \subset Q}} \mathcal{C}(S)\right) .
$$

Our first collection of cubes for $\nu^{+}$is $\tilde{\Delta}^{0}=\left\{R(Q): Q \in \Delta^{0}\right\}$, which we naturally split into the $\tilde{\Delta}_{k}^{0}=\left\{R(Q): Q \in \Delta^{0} \cap \Delta_{k}\right\}, k \geq 0$. We need to complete $\tilde{\Delta}^{0}$ with cubes that come from decomposing the circles $\mathcal{C}(Q)$, $Q \in I_{1} \cup L I$.

For each cube $Q \in I_{1} \cup L I$ we construct a collection $\tilde{\Delta}(Q)$ of subsets of $\mathcal{C}(Q)$ as follows. We start at generation $k(Q)+1$; we cut $\mathcal{C}(Q)$ into (disjoint) arcs of circle of equal length $\ell_{1}$, with $10 A^{-k(Q)-1} \leq \ell_{1} \leq$ $20 A^{-k(Q)-1}$, say, and call $\tilde{\Delta}_{k(Q)+1}(Q)$ the collection of these arcs of circle. Then we subdivide further each arc $R \in \tilde{\Delta}_{k(Q)+1}(Q)$ into smaller arcs of circle of equal length $\ell_{2} \in\left[10 A^{-k(Q)-2}, 20 A^{-k(Q)-2}\right]$, and call $\tilde{\Delta}_{k(Q)+2}(Q)$ the resulting collection of arcs of $\mathcal{C}(Q)$. We continue like this, and eventually construct a collection $\tilde{\Delta}_{k}(Q)$ of (disjoint) subarcs of $\mathcal{C}(Q)$ for all $k>k(Q)$, and with the usual properties of dyadic cubes. Finally set $\tilde{\Delta}(Q)=\bigcup_{k>k(Q)} \tilde{\Delta}_{k}(Q)$. 
Our collection of cubes for $\nu^{+}($and $\nu)$ is

$$
\tilde{\Delta}=\tilde{\Delta}^{0} \cup\left(\bigcup_{Q \in I_{1} \cup L I} \tilde{\Delta}(Q)\right)
$$

which we can decompose into the

$$
\tilde{\Delta}_{k}=\tilde{\Delta}_{k}^{0} \cup\left(\bigcup_{\substack{Q \in I_{1} \cup L I \\ k(Q)<k}} \tilde{\Delta}_{k}(Q)\right) .
$$

First we want to check that $\tilde{\Delta}$ has the combinatorial properties (3.2) and (3.3). We start with the first one:

$$
\begin{aligned}
& \text { for each } k \geq 0, \tilde{E} \text { is the disjoint } \\
& \text { union of the cubes } R, R \in \tilde{\Delta}_{k} .
\end{aligned}
$$

Fix $k \geq 0$. Because $E_{\infty}$ does not meet the cubes of $I_{1} \cup L I$ (see after (10.27)), it does not meet the cubes of $\Delta \backslash \Delta^{0}$ either (by definition (10.14)), and then (11.5) says that $E_{\infty}$ is the disjoint union of the $E_{\infty} \cap R(Q), Q \in \Delta_{k}^{0}$. So we are left with the circles $\mathcal{C}(S), S \in I_{1} \cup L I$. If $S \in I_{1} \cup L I$ and $k(S) \geq k$, then there is exactly one cube $Q \in \Delta_{k}^{0}$ that contains $S$, and $\mathcal{C}(S)$ is contained in $R(Q)$ by (11.5). Moreover $\mathcal{C}(S)$ does not meet any other $R\left(Q^{\prime}\right), Q^{\prime} \in \Delta_{k}^{0}$, and it does not meet any of the circles $\mathcal{C}\left(Q^{\prime \prime}\right), Q^{\prime \prime} \in I_{1} \cup L I$ and $k\left(Q^{\prime \prime}\right)<k$ (and even less the corresponding cubes of $\left.\tilde{\Delta}_{k}\left(Q^{\prime \prime}\right)\right)$. Thus the cubes of $\tilde{\Delta}_{k}$ partition $\mathcal{C}(S)$. If $k(S)<k$, then $\mathcal{C}(S)$ does not meet any of the $R(Q), Q \in \Delta_{k}^{0}$, because all the circles contained in those circles come from cubes $Q^{\prime}$ with $k\left(Q^{\prime}\right) \geq k>k(S)$. It does not meet the $\tilde{\Delta}_{k}\left(S^{\prime}\right), S^{\prime} \neq S$, either, and it is nicely covered by the cubes of $\tilde{\Delta}_{k}(S)$. This completes our proof of (11.8).

Next we check (3.3). Let $R_{1} \in \tilde{\Delta}_{k}$ and $R_{2} \in \tilde{\Delta}_{k+1}$ be given, and suppose that $R_{1} \cap R_{2} \neq \varnothing$. If $R_{1} \cap R_{2} \cap E_{\infty} \neq \varnothing$, then $R_{1}=R\left(Q_{1}\right)$ and $R_{2} \in R\left(Q_{2}\right)$ for cubes $Q_{1} \in \Delta_{k}$ and $Q_{2} \in \Delta_{k+1}$, and (11.5) says that $Q_{1} \cap Q_{2} \supset R_{1} \cap R_{2} \cap E_{\infty} \neq \varnothing$. Then $Q_{2} \subset Q_{1}$ and $R_{2} \subset R_{1}$. If $R_{1} \cap R_{2} \cap E_{\infty}=\varnothing$, then $R_{1} \cap R_{2} \cap \mathcal{C}(S) \neq 1$ for some $S \in I_{1} \cup L I$. If $k>k(S)$, then $R_{1}, R_{2} \subset \tilde{\Delta}(S)$ and $R_{2} \subset R_{1}$ by construction of $\tilde{\Delta}(S)$. If $k=k(S)$, then $R_{1}=R(S)$ and $R_{2} \in \tilde{\Delta}(S)$, whence $R_{2} \subset R_{1}$. Finally, if $k<k(S)$, then $R_{1}=R\left(Q_{1}\right)$ and $R_{2}=R\left(Q_{2}\right)$ for cubes $Q_{1}, Q_{2} \in \Delta^{0}$ that both contain $S$. In this case also $Q_{2} \subset Q_{1}$ and $R_{2} \subset R_{1}$. This proves (3.3) when $\ell=k+1$; the general case follows because of (11.8). 
Next we want to consider properties of our cubes that involve the measures $\nu$ and $\nu^{+}$. We start with the upper bound for density (3.1)

$$
\nu^{+}(B(x, r)) \leq C r, \quad \text { for all } x \in \mathbb{C} \text { and } r>0
$$

This is proved in [DM], beginning of Section 4.2; unfortunately the statement $(* 2.5)$ only mentions $\nu$ and not $\nu^{+}$, but the proof applies to $\nu^{+}$. (The only difference between $\nu$ and $\nu^{+}$comes from the size of the functions $\rho_{n}$, and the only information used in the proof of $(* 2.5)$ in this respect is that $0 \leq \rho_{n} \leq 1$.)

We also want to relate the measures of our cubes for $\mu, \nu^{+}$, and $\nu$, and to this effect we define numbers $\rho_{Q}, Q \in \Delta^{0}$, by

$$
\rho_{Q}=\prod_{\substack{n \geq 1: Q_{n} \in P L I \\ \text { and } Q \subset G_{n}}}\left(1-\theta_{n}\right)
$$

Recall from (10.12) and (10.14) that if $Q \in \Delta^{0}, Q$ is never strictly contained in a cube of $I_{1} \cup L I$. Let $n_{0}$ denote the largest integer for which $k\left(Q_{n_{0}}\right)<k(Q)$. By construction, the function $\rho_{n_{0}}$ is constant on $Q$, and in fact the only times $\rho_{n}$ has possibly been modified on $Q$ for $n \leq n_{0}$ where when $Q_{n} \in P L I$ and $Q \subset Q_{n}$ (and hence $Q \subset G_{n}$ ). Because of this, the constant value of $\rho_{n_{0}}$ on $Q$ is precisely $\rho_{Q}$ (see (10.18)).

If furthermore $Q \in I_{1}$ and $m$ is the integer such that $Q=Q_{m}$, then $\rho_{m-1}=\rho_{n_{0}}$ on $Q$ because the cubes $Q_{\ell}, n_{0}<\ell<m$, do not meet $Q$. (All these cubes lie in $I_{1}$, by definition of our order.) Thus

$$
\rho_{Q_{m}}=\rho_{m}^{*}, \quad \text { when } Q_{m} \in I_{1},
$$

where $\rho_{m}^{*}$ still denotes the constant value of $\rho_{m-1}$ on $Q_{m}$.

If $Q \in L I$ and $m \geq 1$ is such that $Q \in \mathcal{A}_{m}$ (i.e., the parent of $Q$ is $Q_{m}$ ), then $\rho_{m}$ is equal to $\rho_{n_{0}}$ on $Q$, because none of the cubes $Q_{\ell}$, $m<\ell<n_{0}$ meet $Q_{m}$. Thus

$$
\rho_{Q}=\rho_{m}^{*}, \quad \text { when } Q \in \mathcal{A}_{m} \text {. }
$$

(We just proved this when $Q_{m} \in P L I$, but (11.11) says that this is also true when $Q_{m} \in I_{1}$.) 
Lemma 11.13. For all $Q \in \Delta^{0}$,

$$
\nu(R(Q)) \leq \rho_{Q} \nu^{+}(R(Q)) \leq \rho_{Q} \mu(Q) \leq C \nu(R(Q)) .
$$

We start with the first inequality. Let us even prove that for all $Q \in \Delta^{0}$,

$$
d \nu \leq \rho_{Q} d \nu^{+}, \quad \text { on } R(Q)
$$

Recall that $\rho_{Q}$ is the constant value on $Q$ of $\rho_{n_{0}}$, where $n_{0}$ denotes the largest integer such that $k\left(Q_{n_{0}}\right)<k(Q)$. Obviously $\rho_{\infty} \leq \rho_{n_{0}}=\rho_{Q}$ on $Q$, and hence $\rho_{\infty} d \mu \leq \rho_{Q} \mathbf{1}_{E_{\infty}} d \mu$ on $E_{\infty} \cap Q=E_{\infty} \cap R(Q)$. Thus $d \nu \leq \rho_{Q} d \nu^{+}$on $E_{\infty} \cap R(Q)$ (see the definitions (10.30) and (11.1) of $\nu$ and $\nu^{+}$). Now let $\mathcal{C}(S)$ be one of the circles that compose $R(Q)$, as in (11.5). Let $n$ denote the integer such that $S \in \mathcal{A}_{n}$. Then $d \nu=d \nu_{n}=$ $\rho_{n}^{*} d \nu_{n}^{+}=\rho_{n}^{*} d \nu^{+}$on $\mathcal{C}(S)$, by (11.2). Since $\rho_{n}^{*}=\rho_{S}$ by (11.12), $S \subset Q$ by (11.5), and $\rho_{Q}$ is obviously a nondecreasing function of $Q$, we get that $\rho_{n}^{*} \leq \rho_{Q}$ and $d \nu \leq \rho_{Q} d \nu^{+}$on $\mathcal{C}(S)$. This proves (11.15).

The second inequality in (11.14) is fairly straightforward

$$
\begin{aligned}
\nu^{+}(R(Q)) & =\mu\left(Q \cap E_{\infty}\right)+\sum_{\substack{S \in I_{1} \cup L I \\
S \subset Q}} \nu^{+}(\mathcal{C}(S)) \\
& =\mu\left(Q \cap E_{\infty}\right)+\sum_{\substack{S \in I_{1} \cup L I \\
S \subset Q}} \mu(S) \\
& \leq \mu(Q),
\end{aligned}
$$

by (11.5), (11.2), (10.12), and the fact that $E_{\infty}$ does not meet the cubes of $I_{1} \cup L I$.

To prove the last inequality, we want to use the fact that the integral of $g d \nu$ on $Q$ is not too small. Let us first check that

$$
\operatorname{Re} \int_{Q} f d \mu>a_{0} \mu(Q), \quad \text { for all } Q \in \Delta^{0} \backslash L I,
$$

where $a_{0}<a_{1}$ is some positive constant (the same one as in [DM].) When $Q \in \Delta^{0} \backslash\left(I_{1} \cup L I\right)$, this follows directly from (10.13), the definition (10.14) of $\Delta^{0}$, and the fact that $a_{0}<a_{1}$. When $Q \in I_{1}, Q$ is not contained strictly in any cube of $H D \cup M I$ (see the definition of $I_{1}$ in 
[DM], just above $(* 4.11)$ ), because it is a maximal cube of $H D \cup M I$. Also, $Q$ is not contained in any cube of $L I$ (by (10.12), or the definition of $I_{1}$ ). Since $L I$ is (by definition) the set of maximal cubes with the properties that

$$
Q \text { is not strictly contained in any cube of } H D \cup M I
$$

and that (11.17) does not hold (see $(* 4.8)$ and $(* 4.9)$ ), and since we know already that $Q$ satisfies (11.18), we get that it satisfies (11.17), as promised.

Let $Q \in \Delta^{0} \backslash L I$ be given, and again denote by $n_{0}$ the largest integer such that $k\left(Q_{n_{0}}\right)<k(Q)$. Observe that $Q$ does not meet any of the $\mathcal{C}_{n}, n \leq n_{0}$; otherwise $Q$ would meet a cube of $\mathcal{A}_{n}$, thus would be contained in this cube (because $k\left(Q_{n}\right)<k(Q)$ ), and even would be strictly contained in it (because $k\left(Q_{n}\right)<k(Q)$ if $Q_{n} \in I_{1}$ and because $Q \notin L I$ if $\left.Q_{n} \in P L I\right)$, a contradiction with the definition of $\Delta^{0}$. Then

$$
\int_{Q} F_{n_{0}}=\int_{Q} \rho_{n_{0}} f d \mu=\rho_{Q} \int_{Q} f d \mu
$$

by (10.16) and the discussion after (11.10).

Next we claim that

$$
\int_{R(Q)} g d \nu=\int_{Q} F_{n_{0}},
$$

i.e., the further modifications of $F_{n}, n \geq n_{0}$, do not change the integral of $F_{n}$ on (what becomes of) $Q$. This will follow from the fact that

$$
\int_{Q} \rho_{n} f d \mu+\sum_{\substack{1 \leq m \leq n \\ Q_{m} \subset Q}} \alpha_{m}\left\|\nu_{m}\right\|=\int_{Q} F_{n_{0}}
$$

for all $n \geq n_{0}$ by taking limits and comparing with (11.5). (The union of the $\mathcal{C}_{m}, Q_{m} \subset Q$, is the same as the union of the $\mathcal{C}(S), S \in I_{1} \cup L I$ and $S \subset Q$, because $Q \notin L I$.) The relation (11.21) is easily proved by induction. It holds for $n_{0}$ because no $Q_{m}, m \leq n_{0}$, can be contained in $Q$ (they are all of strictly earlier generations). If (11.21) holds for $n-1, n>n_{0}$, and if $Q_{n}$ does not meet $Q$, then (11.21) also holds for $n$ because the left-hand side is not modified. Otherwise, $Q_{n} \subset Q$ (because $k\left(Q_{n}\right) \geq k(Q)$ ), and all the modifications of the integral of $F_{n-1}$ affect 
the left-hand side of (11.21). Since the sum of these modifications is zero by (10.23) (or by construction), (11.21) for $n$ follows from (11.21) for $n-1$.

From (11.17), (11.19) and (11.20) we deduce that

$$
\begin{aligned}
a_{0} \rho_{Q} \mu(Q) & \leq \rho_{Q} \operatorname{Re} \int_{Q} f d \mu \\
& \leq \rho_{Q}\left|\int_{Q} f d \mu\right| \\
& \leq\left|\int_{Q} F_{n_{0}}\right| \\
& =\left|\int_{R(Q)} g d \nu\right| \\
& \leq C \nu(R(Q))
\end{aligned}
$$

because $g$ is bounded (by (10.31) and (10.25)). This proves the last inequality in (11.14) when $Q \in \Delta^{0} \backslash L I$.

When $Q \in L I, R(Q)=\mathcal{C}(Q)$ and $\nu(R(Q))=\nu(\mathcal{C}(Q))=\rho_{n}^{*} \mu(Q)$, where $n$ is such that $Q \in \mathcal{A}_{n}$ and $\rho_{n}^{*}$ is as in (10.20). Thus (11.12) says that $\nu(R(Q))=\rho_{Q} \mu(Q)$, and (11.14) holds in this case as well. Lemma 11.13 follows.

Note that (11.14) implies that $\nu(R)>0$ for all $R \in \tilde{\Delta}^{0}$, because $\mu(Q)>0$ for all $Q \in \Delta$. (Recall that $Q$ is centered on $E=\operatorname{supp} \mu$.) Thus $\nu(R)>0$ for all $R \in \tilde{\Delta}$, and so $\tilde{E} \subset \operatorname{supp} \nu \subset \operatorname{supp} \nu^{+}$. (We shall see soon that $\operatorname{diam} R \leq C A^{-k}$ for $R \in \tilde{\Delta}_{k}$.) As was observed in Remark 3.27, this and (11.4) are just as good, in view of Theorem 3.20, as knowning that $\tilde{E}=\operatorname{supp} \nu$ or $\tilde{E}=\operatorname{supp} \nu^{+}$.

We want to continue checking that $\nu^{+}, \tilde{E}$, and $\tilde{\Delta}$ satisfy the hypotheses for Theorem 3.20. We already know that (3.1)-(3.3) hold, and the next verification in our list is the story about the balls $B(Q)$.

Thus we want to define a center $x(R)$ and a radius $r(R)$ for every $R \in \tilde{\Delta}$. We start with the case when $R \in \tilde{\Delta}^{0}$ and $R=R(Q)$ for some $Q \in \Delta^{0}$. First,

$$
\operatorname{dist}(x(Q), R) \leq \frac{r(Q)}{100} .
$$

Indeed, if $x(Q)$ does not lie in $E_{\infty}$, there are only two possibilities. The first one is that $x(Q) \in Q^{\prime}$ for some $Q^{\prime} \in I_{1} \cup L I$ which is contained 
in $Q$. If $Q^{\prime}=Q$, then (11.23) holds because $R=\mathcal{C}(Q)$. If $Q^{\prime}$ is strictly contained in $Q$ (i.e., of a strictly later generation), then

$$
\operatorname{dist}(x(Q), R) \leq \operatorname{dist}\left(x(Q), \mathcal{C}\left(Q^{\prime}\right)\right) \leq 60 r\left(Q^{\prime}\right) \leq \frac{r(Q)}{100} .
$$

The second possibility is that $\rho_{n}(x(Q))$ tends to 0 without ever being equal to 0 . Indeed, $0 \leq \theta_{n}<1$ for all $n$, and hence (10.18) says that the only places where $\rho_{n}$ becomes 0 are the $H_{n}$ 's, i.e., the cubes of $I_{1} \cup L I$. In this second case $x(Q)$ lies in infinitely many cubes $Q_{n} \in P L I$, and dist $(x(Q), R)=0$. Thus (11.23) holds in all cases.

Let us also check that

$$
\begin{aligned}
& \text { every point of } R=R(Q) \text { lies at distance } \\
& \text { less or equal than } \frac{r(Q)}{100} \text { from } Q .
\end{aligned}
$$

Of course there is nothing to check for points of $Q \cap E_{\infty}$; thus we are left with points of the circles $\mathcal{C}(S), S \in I_{1} \cup L I$ and $S \subset Q$ (see (11.5)). These points are within $r(S) / 100$ of some center $x(S) \in Q$, by definition of $\mathcal{C}(S)$; (11.24) follows because $r(S) \leq r(Q)$ when $S \subset Q$.

Let us choose a center $x(R) \in R$ at distance at most $r(Q) / 100$ from $x(Q)$ and take $r(R)=r(Q)$. Then (3.4) is the same as (10.3), and

$$
R \subset \tilde{E} \cap B(x(R), 29 r(R)),
$$

by (10.4) and (11.24). Let us also verify that

$$
\tilde{E} \cap B\left(x(R), \frac{98 r(R)}{100}\right) \subset R .
$$

Let $x \in \tilde{E} \cap B(x(R), 98 r(R) / 100)$ be given. If $x \in E_{\infty}$, then $x \in Q$ by (10.4), and hence $x \in R$. Otherwise $x \in \mathcal{C}(S)$ for some $S \in I_{1} \cup L I$. If $S \subset Q$ we are happy because then $\mathcal{C}(S) \subset R$ by (11.5). So let us assume this is not the case. Then $S \cap Q=\varnothing$, because $Q$ cannot be strictly contained in $S$ (since $Q \in \Delta^{0}$ ). We know that

$$
\operatorname{dist}(x, Q) \leq|x-x(Q)|<\frac{99}{100} r(Q) \text {, }
$$

but on the other hand (10.28) says that

$$
\operatorname{dist}(x, Q) \geq \operatorname{dist}(\mathcal{C}(S), Q) \geq \operatorname{dist}(\mathcal{C}(S), E \backslash S) \geq \frac{99}{100} r(S),
$$


and so $r(S)<r(Q)$. Then

$$
\operatorname{dist}(x, S) \leq \frac{r(S)}{100}<\frac{r(Q)}{100},
$$

and there are points of $S$ at distance less than $|x-x(Q)|+r(Q) / 100 \leq$ $r(Q)$ from $x(Q)$. This is impossible because of (10.4), and (11.26) follows. (Note that the argument did not need to be as tight as it looks, because in the dangerous case where $r(S) \sim r(Q)$, we could use (10.5) to get a somewhat more brutal contradiction.)

Our estimates (11.25) and (11.26) are not quite the same as (3.5), because of the factor $98 / 100$, but they are just as good for the proof of Theorem 3.20. We could also have decided to take $r(R)=98 r(Q) / 100$; then we would have obtained (3.5), but only

$$
\frac{98}{100} A^{-k} \leq r(Q) \leq C_{1} A^{-k}
$$

instead of (3.4). This difference is even more obviously harmless (just dilate $E$.)

We still need to define $x(R)$ and $r(R)$ when $R \in \tilde{\Delta} \backslash \tilde{\Delta}^{0}$, i.e., when $R \in \tilde{\Delta}_{k}(Q)$ for some $Q \in I_{1} \cup L I$ and some $k>k(Q)$. In this case $R$ is a small arc of the circle $\mathcal{C}(Q)$, with length $\ell \in\left[10 A^{-k}, 20 A^{-k}\right]$. We choose for $x(R)$ the center of this arc and take $r(Q)=A^{-k}$. Then (11.25) and (11.26) (and even the analogue of (3.5)) hold for $R$ because $k>k(Q)$ and

$$
\operatorname{dist}(\mathcal{C}(Q), \tilde{E} \backslash \mathcal{C}(Q)) \geq \frac{98}{100} r(Q), \quad \text { for all } Q \in I_{1} \cup L I,
$$

by (10.28) and (10.29).

This completes our discussion of (3.4) and (3.5). Since (3.6) is the same as (10.6), we are left with the story about small boundaries. We first need to define numbers $\xi(R), R \in \tilde{\Delta}$.

When $R \in \tilde{\Delta}(Q)$ for some $Q \in I_{1} \cup L I$, simply take $\xi(R)=\nu^{+}(R)$. When $R \in \tilde{\Delta}^{0}$, set $\xi(R)=\mu\left(10^{4} B(Q)\right)$, where $Q \in \Delta^{0}$ is such that $R=R(Q)$. Let us first check the auxiliary conditions (3.9)-(3.12), and then we shall return to (3.8).

When $R \in \tilde{\Delta}(Q),(11.27)$ and the fact that $k(R)>k(Q)$ imply that $\tilde{E} \cap 91 B(R)=\mathcal{C}(Q) \cap 91 B(R)$. The property (3.9) for $R$ and the measure $\nu^{+}$follows from the fact that $\nu^{+}$is a bounded constant times Hausdorff measure on $\mathcal{C}(Q)$ (by (11.2) and (10.15)); (3.10) for $R$ follows 
because in addition $\xi(S)=\nu^{+}(S)$ for all the cubes $S \in \tilde{\Delta}(Q)$. All cubes of $\tilde{\Delta}(Q)$ are good for $\nu^{+}$(i.e., satisfy (3.11) for $\nu^{+}$), and hence we don't need to check (3.12) for them.

Now consider $R \in \tilde{\Delta}^{0}$, and let $Q \in \Delta^{0}$ be such that $R=R(Q)$. Recall that we chose $r(R)=r(Q)$ and $x(R)$ at distance less or equal than $r(Q) / 100$ from $x(Q)$. (See above $(11.25)$ ). Thus $91 B(R) \subset 92 B(Q)$.

Let $\mathcal{A}$ denote the set of cubes $S \in I_{1} \cup L I$ such that $\mathcal{C}(S)$ meets $91 B(R)$. Then

$$
\begin{aligned}
\nu^{+}(91 B(R)) & \leq \nu^{+}\left(E_{\infty} \cap 91 B(R)\right)+\sum_{S \in \mathcal{A}} \nu^{+}(\mathcal{C}(S)) \\
& \leq \mu\left(E_{\infty} \cap 91 B(R)\right)+\sum_{S \in \mathcal{A}} \mu(S) \\
& \leq \xi(R)+\sum_{S \in \mathcal{A}} \mu(S)
\end{aligned}
$$

by (11.3), (11.2), the facts that $\nu^{+} \leq \mu$ on $E_{\infty}$ and $91 B(R) \subset 92 B(Q)$, and the definition of $\xi(R)$. If $S \in \mathcal{A}$ and $S$ is not contained in $Q$, then $S \cap Q=\varnothing$ because $Q$ cannot be strictly contained in $S$, since $Q \in \Delta^{0}$. Then (10.28) says that

$$
r(S) \leq \frac{100}{99} \operatorname{dist}(\mathcal{C}(S), E \backslash S) \leq \frac{100}{99} \operatorname{dist}(\mathcal{C}(S), x(Q)) \leq 100 r(Q) .
$$

Then (10.4) says that $S \subset 10^{4} B(Q)$. Hence

$$
\sum_{S \in \mathcal{A}} \mu(S) \leq \mu\left(10^{4} B(Q)\right)=\xi(R)
$$

and (3.9) follows from (11.28) and (10.15).

Now fix $k>k(R)=k(Q)$, and denote by $\mathcal{B}_{k}$ the set of cubes $T \in \Delta_{k}^{0}$ such that $R(T) \subset 91 B(R)$. If $T \in \mathcal{B}_{k}, T \subset 93 B(Q)$, by crude estimates on $\operatorname{diam}(T \cup R(T))$ and the fact that $k>k(Q)$. Then

$$
\begin{aligned}
\sum_{T \in \mathcal{B}_{k}} \xi(R(T)) & =\sum_{T} \mu\left(10^{4} B(T)\right) \\
& \leq C \mu\left(\bigcup_{T}\left(10^{4} B(T)\right)\right) \\
& \leq C \xi(R)
\end{aligned}
$$

because the $10^{4} B(T), T \in \Delta_{k}$, have bounded overlap and are contained in $10^{4} B(Q)$. This takes care of the cubes of $\tilde{\Delta}^{0}$ in the sum in (3.10). Now 
let $\mathcal{D}_{k}$ be the set of cubes $T \in \tilde{\Delta}_{k} \backslash \tilde{\Delta}^{0}$ that are contained in $91 B(R)$. All these cubes lie in sets $\tilde{\Delta}(S)$ for cubes $S \in I_{1} \cup L I$ such that $\mathcal{C}(S)$ meets $91 B(R)$. Hence

$$
\sum_{T \in \mathcal{D}_{k}} \xi(T)=\sum_{T} \nu^{+}(T) \leq \nu^{+}\left(\bigcup_{S \in \mathcal{A}} \mathcal{C}(S)\right)=\sum_{S \in \mathcal{A}} \mu(S) \leq \xi(R)
$$

because the cubes $T \in \mathcal{D}_{k}$ are disjoint, and by the discussion above. This completes the verification of (3.10) for $R \in \tilde{\Delta}^{0}$.

Finally (3.11)-(3.12) follows easily from its counterpart (10.9)(10.11) if $C_{0} \geq C_{1}$, and also the only cube of $\tilde{\Delta}_{0}$ is good for $\nu^{+}$and (3.11) because the only cube of $\Delta_{0}$ is good for (10.9) or $(* 3.14)$.

We still need to check (3.8) for cubes of $\tilde{\Delta}$. For cubes $R \in \tilde{\Delta}(Q)$, $Q \in I_{1} \cup L I$, this follows from the fact that $N_{t}(R) \subset \mathcal{C}(Q)$, by (11.27), and the simple structure of the cubes of $\tilde{\Delta}(Q)$.

Now let $R \in \tilde{\Delta}^{0}$ be given, and let $Q \in \Delta^{0}$ be such that $R=R(Q)$. Also set $k=k(R)=k(Q)$ and

$$
\begin{aligned}
N_{t}= & \left\{x \in R: \operatorname{dist}(x, \tilde{E} \backslash R) \leq t A^{-k}\right\} \\
& \cup\left\{x \in \tilde{E} \backslash R: \operatorname{dist}(x, R) \leq t A^{-k}\right\},
\end{aligned}
$$

for $0 \leq t \leq 1$. This is the set that we need to control for (3.8). Still denote by $N_{t}(Q)$ the set in (10.7); we want to use (10.8) to control the sets $N_{t}$. Note that because of (3.9), it is enough to prove that

$$
\nu^{+}\left(N_{t}\right) \leq C t^{\tau} \xi(Q)=C t^{\tau} \mu\left(10^{4} B(Q)\right)
$$

for $0<t \leq 10^{-2}$, say.

So let $0<t \leq 10^{-2}, y \in R \cap N_{t}$, and $z \in N_{t} \backslash R$ be given, with $|y-z|<2 t A^{-k}$. Note that for each $y \in R \cap N_{t}$ there is a $z$ like this, and for each $z \in N_{t} \backslash R$ there is an $y$ like this. Let us distinguish a few cases.

If $y$ and $z$ both lie in $E_{\infty}$, then $y \in Q$ and $z \in E \backslash Q$, and so $y$ and $z$ both lie in $N_{2 t}(Q)$.

Next consider the case when $z \in E_{\infty}$ (and hence $z \in E \backslash Q$ ) and $y \in R \backslash E_{\infty}$. Then (11.5) says that $y \in \mathcal{C}(S)$ for some $S \in I_{1} \cup L I$ such that $S \subset Q$, and

$$
2 t A^{-k} \geq|y-z| \geq \operatorname{dist}(\mathcal{C}(S), E \backslash Q) \geq \frac{99}{100} r(S)
$$


by (10.28). The center $x(S)$ of $S$ lies in $S \subset Q$, while $z \in E \backslash Q$; since

$$
|x(S)-y|+|y-z| \leq \frac{r(S)}{100}+2 t A^{-k}<3 t A^{-k},
$$

we get that $z$ and $x(S)$ lie in $N_{3 t}(Q)$. Using (11.33) again and (10.4), we deduce from this that the whole cube $S$ lies in $N_{100 t}(Q)$.

Our next case is when $y \in R \cap E_{\infty}=Q \cap E_{\infty}$ and $z \in(\tilde{E} \backslash R) \backslash E_{\infty}$. Then (11.3) says that $z \in \mathcal{C}(S)$ for some $S \in I_{1} \cup L I$, and (11.5) even adds that $S$ is not contained in $Q$. Moreover $S \cap Q=\varnothing$, because $Q$ cannot be strictly contained in $S$ (since $Q \in \Delta^{0}$ ). This time

(11.34) $2 t A^{-k}>|y-z|>\operatorname{dist}(\mathcal{C}(S), Q) \geq \operatorname{dist}(\mathcal{C}(S), E \backslash S) \geq \frac{99}{100} r(S)$,

by (10.28), and

$$
|x(S)-y| \leq|x(S)-z|+|z-y| \leq \frac{r(S)}{100}+2 t A^{-k}<3 t A^{-k} .
$$

Since $y \in Q$ and $x(S) \in S \subset E \backslash Q$, we get that $y \in N_{3 t}(Q), x(S) \in$ $N_{3 t}(Q)$, and (by (10.4) and (11.34)) the whole $S$ lies in $N_{100 t}(Q)$.

Our last case is when $y$ and $z$ lie in $\tilde{E} \backslash E_{\infty}$. Then $y \in \mathcal{C}(S)$ for some $S \in I_{1} \cup L I$ such that $S \subset Q$, and $z$ lies in $\mathcal{C}(T)$ for some $T \in I_{1} \cup L I$ such that $T \cap Q=\varnothing$. Then

(11.35) $2 t A^{-k}>|y-z| \geq \operatorname{dist}(\mathcal{C}(S), \mathcal{C}(T)) \geq \frac{98}{100} \max \{r(S), r(T)\}$,

by (10.29). Since $x(S) \in S \subset Q$ and $x(T) \in T \subset E \backslash Q$, and

$$
|x(S)-x(T)| \leq|y-z|+\frac{r(S)}{100}+\frac{r(T)}{100}<3 t A^{-k},
$$

we get that $x(S), x(T) \in N_{3 t}(Q)$, and then that $S$ and $T$ are contained in $N_{100 t}(Q)$ (by (11.35) again.)

We may now summarize our discussion:

$$
N_{t} \subset\left(E_{\infty} \cap N_{3 t}(Q)\right) \cup\left(\bigcup_{S \in Z} \mathcal{C}(S)\right),
$$

where $Z$ denotes the set of cubes $S \in I_{1} \cup L I$ that are contained $N_{100 t}(Q)$. Now

$$
\begin{aligned}
\sum_{S \in Z} \nu^{+}(\mathcal{C}(S)) & =\sum_{S \in Z} \mu(S) \\
& \leq 100 C_{2} t^{\tau} \mu(90 B(Q)) \\
& \leq 100 C_{2} t^{\tau} \xi(R),
\end{aligned}
$$


by (11.2), (10.12), (10.8), and the definition of $\xi(R)$. Since $\nu^{+}\left(E_{\infty} \cap\right.$ $\left.N_{3 t}(Q)\right) \leq 3 C_{2} t^{\tau} \xi(R)$ by (10.8) again, (11.32) follows from (11.36) and (11.37).

This completes our verification of the hypotheses of Theorem 3.20 for the set $\tilde{E}$, the measure $\nu^{+}$, and the cubes of $\tilde{\Delta}$. In the next section we use this information to show that Theorem 3.20 also holds on $\tilde{E}, \nu$, and with the cubes of $\tilde{\Delta}$, even though the hypotheses (3.8)-(3.12) are not necessarily satisfied in this case.

\section{Theorem 3.20 holds for $\nu$.}

In general we do not expect that $\nu$ (equipped with the cubes of $\tilde{\Delta})$ will satisfy the conditions (3.8)-(3.12) about small boundaries. A typical bad thing that may happen is the following. For some good cubes $R=R(Q), Q \in \Delta^{0}$, the factor $\rho_{Q}$ in (11.14) could be very small, much smaller than the corresponding factors for other cubes that touch $R$. When this happens, we shall not have a good control on the measure for $\nu$ of the sets $N_{t}(R)$ in terms of $\nu(R)$, and so we may have to declare that $R$ is bad for $\nu$ without having any compensation available in terms of (3.12). Nonetheless we want to prove that Theorem 3.20 holds for $\tilde{E}, \nu$, and the cubes of $\tilde{\Delta}$.

By this we mean that if $T: b \mathcal{E} \times b \mathcal{E} \longrightarrow \mathbb{C}$ is an operator that satisfies (3.13)-(3.15) and (3.17) (with $\mu$ and $\Delta$ replaced with $\nu$ and $\tilde{\Delta})$, and if there are functions $\beta, \tilde{\beta} \in \operatorname{BMO}(d \nu)$ that satisfy (3.21) and (3.22) (for $\nu$ ), then $T$ extends to a bounded operator on $L^{2}(d \nu)$. The definition of $\operatorname{BMO}(d \nu)$ is the same as for $d \mu$ : we do not use small boundaries there.

To prove our claim, we shall simply follow the proof of Theorem 3.20 and show that it applies.

All the arguments in sections 2-7 can be applied without modification; the small boundary properties are never used there, except to get qualitative information like (3.16) or (7.1). These properties are also true for $\nu$ because they hold for $\nu^{+}$. Thus we can get as far as Lemma 7.40, which says that it is enough to prove that the matrix $\mathcal{N}$ (associated to the measure $\nu$ ) defines a bounded operator on $\ell^{2}(\tilde{\Delta})$.

We already know from Section 11 that the corresponding matrix $\mathcal{N}^{+}$for $\nu^{+}$defines a bounded operator, and so it will be enough to show that

$$
N(Q, R) \leq C N^{+}(Q, R)
$$


(with obvious notations). To make the comparison easier, it will be useful to define positive numbers $\rho_{R}$ for all $R \in \tilde{\Delta}$. When $R \in \tilde{\Delta}^{0}$ and $R=R(Q)$ for some $Q \in \Delta^{0}$, we take $\rho_{R}=\rho_{Q}$. When $R \in \tilde{\Delta}(Q)$ for some $Q \in I_{1} \cup L I$, we set $\rho_{R}=\rho_{Q}$. We claim that

$$
d \nu \leq \rho_{R} d \nu^{+}, \quad \text { on } R
$$

and

$$
\nu(R) \geq C^{-1} \rho_{R} \nu^{+}(R), \quad \text { for all } R \in \tilde{\Delta} .
$$

When $R \in \tilde{\Delta}^{0}$ and $R=R(Q)$, this follows from (11.15) and (11.14). When $R \in \tilde{\Delta}(Q)$ for some $Q \in I_{1} \cup L I$, this is obvious because $\nu=$ $\rho_{Q} \nu^{+}$on $\mathcal{C}(Q)$, by (11.2) and (11.12).

We are now ready to prove (12.1). We shall just take the different types of coefficients $N(Q, R)$ from (7.36)-(7.39) one after the other and compare them with the corresponding ones for $\nu^{+}$. We start with $A_{1}(Q, R)$ in (7.13). Recall that $A_{1}(Q, R)$ is a sum of terms

$$
\left(\nu\left(Q^{*}\right) \nu\left(R^{*}\right)\right)^{-1 / 2} I\left(Q^{*}, R^{*} \cap 2 Q\right),
$$

where $Q^{*} \in F(Q)$ (the set of children of $Q$ ) and $R^{*} \in F(R)$. Note that for each choice of $Q^{*}$ and $R^{*}$,

$$
\left(\nu\left(Q^{*}\right) \nu\left(R^{*}\right)\right)^{-1 / 2} \leq C\left(\rho_{Q^{*}} \rho_{R^{*}} \nu^{+}\left(Q^{*}\right) \nu^{+}\left(R^{*}\right)\right)^{-1 / 2},
$$

by (12.3), and

$$
\begin{aligned}
I\left(Q^{*}, R^{*} \cap 2 Q\right) & =\int_{Q^{*}} \int_{R^{*} \cap 2 Q} \frac{d \nu(x) d \nu(y)}{|x-y|} \\
& \leq \rho_{Q^{*}} \rho_{R^{*}} I^{+}\left(Q^{*}, R^{*} \cap 2 Q\right)
\end{aligned}
$$

by (12.2). Here we set

$$
I^{+}(Q, V)=\int_{Q} \int_{V} \frac{d \nu^{+}(x) d \nu^{+}(y)}{|x-y|}
$$

for $Q \in \tilde{\Delta}$ and $V \subset \tilde{E} \backslash Q$, the obvious analogue of $I(Q, V)$ for $\nu^{+}$.

From (12.4) and (12.5) we deduce that $A_{1}(Q, R) \leq C A_{1}^{+}(Q, R)$ (with obvious notations). 
Next let $A_{2}(Q, R)$ be as in (7.14);

$$
\begin{aligned}
A_{2}(Q, R) & =\nu(Q)^{1 / 2} \sum_{R^{*} \in F(R)} \nu\left(R^{*}\right)^{-1 / 2} J\left(Q, R^{*} \backslash 2 Q\right) \\
& \leq C \nu^{+}(Q)^{1 / 2} \sum_{R^{*}} \rho_{R^{*}}^{-1 / 2} \nu^{+}\left(R^{*}\right)^{-1 / 2} J\left(Q, R^{*} \backslash 2 Q\right) \\
& \leq C \nu^{+}(Q)^{1 / 2} \sum_{R^{*}} \rho_{R^{*}}^{1 / 2} \nu^{+}\left(R^{*}\right)^{-1 / 2} J^{+}\left(Q, R^{*} \backslash 2 Q\right) \\
& \leq C A_{2}^{+}(Q, R)
\end{aligned}
$$

by (12.2), (12.3), (12.2) again, and where $J^{+}$and $A_{2}^{+}(Q, R)$ are the obvious analogous of $J$ and $A_{2}(Q, R)$ for $\nu^{+}$. (See (7.10) for the definition of $J$.)

The story for $A_{3}(Q)$ in $(7.22)$ is similar: $A_{3}(Q)$ is a sum of terms

$$
\nu\left(Q_{1}^{*}\right)^{-1 / 2} \nu\left(Q_{2}^{*}\right)^{-1 / 2} I\left(Q_{1}^{*}, Q_{2}^{*}\right)
$$

$$
\leq C\left(\rho_{Q_{1}^{*}} \rho_{Q_{2}^{*}} \nu^{+}\left(Q_{1}^{*}\right) \nu^{+}\left(Q_{2}^{*}\right)\right)^{-1 / 2} \rho_{Q_{1}^{*}} \rho_{Q_{2}^{*}} I^{+}\left(Q_{1}^{*}, Q_{2}^{*}\right)
$$

and hence $A_{3}(Q) \leq C A_{3}^{+}(Q)$. Next (7.27) says that $B_{11}$ is a sum of terms

$$
\left.\left(\nu\left(Q^{*}\right) \nu\left(R^{*}\right)\right)^{-1 / 2} I\left(Q^{*}, R^{*} \cap 2 Q\right)\right)
$$

$$
\leq C\left(\rho_{Q^{*}} \rho_{R^{*}} \nu^{+}\left(Q^{*}\right) \nu^{+}\left(R^{*}\right)\right)^{-1 / 2} \rho_{Q^{*}} \rho_{R^{*}} I^{+}\left(Q^{*}, R^{*} \cap 2 Q\right)
$$

(still by (12.2) and (12.3)), and hence $B_{11} \leq C B_{11}^{+}$. Similarly $B_{12}$ in (7.28) is composed of terms

$$
\begin{aligned}
& \nu(Q)^{1 / 2} \nu\left(R^{*}\right)^{-1 / 2} J\left(Q, R^{*} \backslash 2 Q\right) \\
& \quad \leq C \nu^{+}(Q)^{1 / 2}\left(\rho_{R^{*}} \nu^{+}\left(R^{*}\right)\right)^{-1 / 2} \rho_{R^{*}} J^{+}\left(Q, R^{*} \backslash 2 Q\right)
\end{aligned}
$$

and is thus $\leq C B_{12}^{+}$. Our next term is $B_{21}$ in (7.29), and it is a sum of terms

$\left(\nu\left(Q^{*}\right) \nu(R(Q))\right)^{-1 / 2} I\left(Q^{*}, 2 Q \backslash R(Q)\right)$

$$
\leq C\left(\rho_{Q^{*}} \rho_{R(Q)} \nu^{+}\left(Q^{*}\right) \nu^{+}(R(Q))\right)^{-1 / 2} \rho_{Q^{*}} I^{+}\left(Q^{*}, 2 Q \backslash R(Q)\right),
$$


which are also dominated by the corresponding terms for $\nu^{+}$because $\rho_{Q^{*}} \leq \rho_{R(Q)}$ (since $Q^{*} \subset Q \subset R(Q)$ by definitions). Finally,

$$
\begin{aligned}
B_{22} & =\nu(Q)^{1 / 2} \nu(R(Q))^{-1 / 2} J(Q, E \backslash(2 Q \cup R(Q))) \\
& \leq \rho_{Q}^{1 / 2} \rho_{R(Q)}^{-1 / 2} B_{22}^{+} \\
& \leq B_{22}^{+}
\end{aligned}
$$

for the same reason.

This completes our verification of (12.1); Theorem 3.20 for $\nu$ and the cubes of $\tilde{\Delta}$ follows.

\section{The Cauchy operator is bounded on $L^{2}(d \nu)$.}

It will be easier for us to deal with the truncated operators $T_{\varepsilon}$, $\varepsilon>0$, defined by

$$
T_{\varepsilon} f(x)=\int_{|x-y|>\varepsilon} \frac{f(y) d \nu(y)}{x-y}, \quad \text { for } f \in L^{2}(d \nu) .
$$

Because $\nu$ is a finite measure, there is no problem in defining $T_{\varepsilon}$, or even in proving that it is a bounded operator on $L^{2}(d \nu)$. Of course we want to prove that $T_{\varepsilon}$ is bounded on $L^{2}(d \nu)$ with bounds that do no depend on $\varepsilon$, and this will require more work.

We cannot apply Theorem 3.20 (for $\nu$ ) directly to $T_{\varepsilon}$, because it does not have a standard kernel, but this will be very easy to fix. Denote by $\mathcal{X}$ the nice cut-off function such that $\mathcal{X}(t)=0$ for $0 \leq t \leq 1 / 2$, $\mathcal{X}(t)=2 t-1$ for $1 / 2 \leq t \leq 1$, and $\mathcal{X}(t)=1$ for $t \geq 1$. Then set

$$
\tilde{T}_{\varepsilon} f(x)=\int \mathcal{X}\left(\frac{|x-y|}{\varepsilon}\right) \frac{f(y) d \nu(y)}{x-y},
$$

for $f \in L^{2}(d \nu)$. We can replace $T_{\varepsilon}$ with $\tilde{T}_{\varepsilon}$ because

$$
\left\|\left|T_{\varepsilon}-\tilde{T}_{\varepsilon}\right|\right\|_{L^{2}(d \nu)} \leq C,
$$

where $\||\cdot|||$ denotes the operator norm, and with a constant $C$ that does not depend on $\varepsilon$. This follows easily from (the continuous version of) Shur's lemma, since

$$
\left|\left(T_{\varepsilon}-\tilde{T}_{\varepsilon}\right) f(x)\right| \leq \int_{\varepsilon / 2<|x-y|<\varepsilon} \frac{|f(y)| d \nu(y)}{|x-y|},
$$


and

$$
\begin{aligned}
& \sup _{x}\left(\int_{\varepsilon / 2<|x-y|<\varepsilon} \frac{d \nu(y)}{|x-y|}\right) \\
& =\sup _{y}\left(\int_{\varepsilon / 2<|x-y|<\varepsilon} \frac{d \nu(x)}{|x-y|}\right) \leq C,
\end{aligned}
$$

by $(11.9)$.

We want to prove that

$$
\left\|\mid T_{\varepsilon}\right\|_{L^{2}(d \nu)} \leq C
$$

with a constant $C$ that does not depend on $\varepsilon$; (13.3) tells us that it is enough to deal with $\tilde{T}_{\varepsilon}$ instead. We want to apply Theorem 3.20 , with $\tilde{E}, \nu$, and the cubes of $\tilde{\Delta}$; Section 12 says that we can do this. We choose $b=g$, where $g$ is as in (10.31). Note that $g$ satisfies (2.6), as was observed shortly after $(10.31)$ (or directly by $(* 2.6)$ ); this was the whole point of the construction in [DM].

The kernel

$$
K(x, y)=\mathcal{X}\left(\frac{|x-y|}{\varepsilon}\right) \frac{1}{x-y}
$$

is antisymmetric and standard with uniform estimates, and $\tilde{T}_{\varepsilon}$ is the singular integral operator associated with $K(x, y)$ as in Lemma 4.8. (Most of the construction is not needed, though, because $K(x, y)$ satisfies the integrability condition (4.2).) In particular, it satisfies the weak boundedness property (3.17) automatically, by antisymmetry. Hence (13.6) will follow as soon as we verify the last condition in Theorem 3.20, namely that $T g$ and $T^{t} g$ lie in BMO with uniform estimates.

Note that we don't need to be as careful as in the statement of Theorem 3.20 and define $\tilde{T} g$ and $\tilde{T}^{t} g$ by duality. Here, due to the fact that our kernel $K$ is bounded, $\tilde{T} g$ and $\tilde{T}^{t} g$ are well defined, and even bounded, and the only thing we have to check is that they lie in BMO with uniform bounds. Also, $\tilde{T} g=-\tilde{T}^{t} g$ by definitions (and in particular antisymmetry), so we only need to show that $\|\tilde{T} g\|_{\mathrm{BMO}(d \nu)} \leq C$ for some $C$ that does not depend on $\varepsilon$.

Note that

$$
\left|\tilde{T}_{\varepsilon} g(x)-T_{\varepsilon} g(x)\right| \leq \int_{\varepsilon / 2<|x-y|<\varepsilon} \frac{|g(y)| d \nu(y)}{|x-y|} \leq C,
$$


by (13.4) and (11.9). Since bounded functions obviously lie in BMO, the desired estimate (13.6) will follow if we prove that

$$
\left\|T_{\varepsilon} g\right\|_{\mathrm{BMO}(d \nu)} \leq C
$$

In view of Definition 3.18, this means that

$$
\int_{R_{0}}\left|T_{\varepsilon} g(x)-m_{R_{0}}\left(T_{\varepsilon} g\right)\right|^{2} d \nu(x) \leq C \nu\left(R_{0}\right)
$$

for all $R_{0} \in \tilde{\Delta}$, where $m_{R_{0}}\left(T_{\varepsilon} g\right)$ denotes the mean value of $T_{\varepsilon} g$ on $R_{0}$ (for $\nu$ ). It is even enough to show that for each $R_{0} \in \tilde{\Delta}$ there is a constant $m_{R_{0}}$ such that

$$
\int_{R_{0}}\left|T_{\varepsilon} g(x)-m_{R_{0}}\right|^{2} d \nu(x) \leq C \nu\left(R_{0}\right),
$$

because we know that the left-hand side of (13.8) is always less than or equal to the left-hand side of (13.9).

Let us first take care of the cubes $R_{0}$ that are contained in circles $\mathcal{C}(Q), Q \in I_{1} \cup L I$.

Lemma 13.10. For each $Q \in I_{1} \cup L I$ there is a constant $C_{Q}^{\varepsilon}$ such that

$$
\left|T_{\varepsilon} g(x)-C_{Q}^{\varepsilon}\right| \leq C, \quad \text { on } \mathcal{C}(Q) \text {. }
$$

Recall that on $\mathcal{C}(Q), g(y)$ is a bounded constant $\alpha_{n}$ (by (10.31) and (10.25)), and $d \nu=\lambda_{Q} d H^{1}$, where $\lambda_{Q}$ is of the form

$$
\rho_{n}^{*} \frac{\mu(Q)}{H^{1}(\mathcal{C}(Q))},
$$

by (10.20). Hence $\lambda_{Q} \leq C$ as well, and

$$
\left|T_{\varepsilon}\left(\mathbf{1}_{\mathcal{C}(Q)} g\right)(x)\right| \leq C,
$$

by elementary properties of truncated Cauchy integrals on circles, and it is enough to study

$$
(13.13) a(x)=T_{\varepsilon}\left(\left(1-\mathbf{1}_{\mathcal{C}(Q)}\right) g\right)(x)=\int_{\{|x-y|>\varepsilon, y \in \tilde{E} \backslash \mathcal{C}(Q)\}} \frac{g(y) d \nu(y)}{x-y} .
$$


Recall from (11.27) that

$$
\operatorname{dist}(\mathcal{C}(Q), \tilde{E} \backslash \mathcal{C}(Q)) \geq \frac{98}{100} r(Q)
$$

so that we can assume that $\varepsilon \geq r(Q) / 2$, say, because otherwise we can replace $\varepsilon$ with $r(Q) / 2$ without modifying $a(x)$. Denote by $x_{0}$ the center of $\mathcal{C}(Q)$, and also set

$$
D=\left\{y \in \tilde{E} \backslash \mathcal{C}(Q):\left|y-x_{0}\right|>\varepsilon\right\}
$$

(the domain of integration for $a\left(x_{0}\right)$ ) and

$$
\mathcal{A}=\left\{y \in \tilde{E}: \varepsilon-\frac{r(Q)}{100} \leq\left|y-x_{0}\right| \leq \varepsilon+\frac{r(Q)}{100}\right\}
$$

(which contains the difference between $D$ and the domain of integration for $a(x)$ when $x \in \mathcal{C}(Q))$. Then

$$
\begin{aligned}
\left|a(x)-a\left(x_{0}\right)\right| \leq & \left|a(x)-\int_{D} \frac{g(y) d \nu(y)}{x-y}\right| \\
& +\left|\int_{D}\left(\frac{1}{x-y}-\frac{1}{x_{0}-y}\right) g(y) d \nu(y)\right| \\
\leq & C \int_{\mathcal{A}} \frac{d \nu(y)}{|x-y|} \\
& +C \int_{\left\{\left|y-x_{0}\right|>r(Q) / 2\right\}}\left|\frac{x-x_{0}}{(x-y)\left(x_{0}-y\right)}\right| d \nu(y) \\
\leq & C,
\end{aligned}
$$

because $\varepsilon \geq r(Q) / 2$, and by the upper density estimate (11.9). (The computation for the last line is the same one as for (8.1).) Thus we can choose $C_{Q}^{\varepsilon}=a\left(x_{0}\right)$, and Lemma 13.10 follows.

Lemma 13.10 immediately gives (13.9) for all the cubes $R_{0}$ that are contained in a $\mathcal{C}(Q)$. Thus we are left with the cubes $R_{0} \in \tilde{\Delta}^{0}$, and we can even suppose that $R_{0}=R\left(Q_{0}\right)$ for some $Q_{0} \in \Delta^{0} \backslash\left(I_{1} \cup L I\right)$. Because of (11.15),

$$
\int_{R_{0}}\left|T_{\varepsilon} g(x)-m_{R_{0}}\right|^{2} d \nu(x) \leq \rho_{Q_{0}} \int_{R_{0}}\left|T_{\varepsilon} g(x)-m_{R_{0}}\right|^{2} d \nu^{+}(x)
$$


and, since $\rho_{Q_{0}} \mu\left(Q_{0}\right) \leq C \nu\left(R_{0}\right)$ by Lemma 11.13, (13.9) will follow if we can show that

$$
\int_{R_{0}}\left|T_{\varepsilon} g(x)-m_{R_{0}}\right|^{2} d \nu^{+}(x) \leq C \mu\left(Q_{0}\right)
$$

Let us summarize what we have done so far.

Lemma 13.17. To prove (13.6) with a constant that does not depend on $\varepsilon$, it is enough to show that for each $\varepsilon>0$ and each cube $Q_{0} \in$ $\Delta^{0} \backslash\left(I_{1} \cup L I\right)$, we can find a complex number $m_{0}$ such that

$$
\int_{R\left(Q_{0}\right)}\left|T_{\varepsilon} g(x)-m_{0}\right|^{2} d \nu^{+}(x) \leq C \mu\left(Q_{0}\right)
$$

where $C$ does not depend on $\varepsilon$ or $Q_{0}$.

At this point we fix a cube $Q_{0}$ as in the lemma, and we want to find $m_{0}$ and eventually check (13.18). Our notations so far have been slightly different from those of [DM, Section 8], where what we call $T_{\varepsilon} g$ was called $T^{\varepsilon}(g d \nu)$. It will be more convenient for us now to revert to the notation of $[\mathrm{DM}]$, i.e., to let the measure show up in the notations. Recall from (10.31), (10.30), and (10.16) that

$$
g d \nu=\lim _{n \rightarrow \infty} F_{n}=f d \mu+\sum_{n \geq 1}\left(F_{n}-F_{n-1}\right)=f d \mu+\sum_{n \geq 1} \varphi_{n}
$$

where

$$
\varphi_{n}=-\mathbf{1}_{H_{n}} \rho_{n-1} f d \mu-\theta_{n} \mathbf{1}_{G_{n}} \rho_{n-1} f d \mu+\alpha_{n} d \nu_{n}
$$

by (10.22). Hence

$$
T^{\varepsilon}(g d \nu)=T^{\varepsilon}(f d \mu)+\sum_{n \geq 1} T^{\varepsilon}\left(\varphi_{n}\right)
$$

the proof of $(* 2.9)$ in $[\mathrm{DM}]$ also gives that the series converges absolutely $\nu^{+}$-almost everywhere, so we should not worry about convergence.

Fortunately we shall not need to estimate most of the terms in (13.21) in the present paper, because this was mostly done in [DM]. 
Denote by $J$ the set of integers $n \geq 1$ such that $Q_{n} \subset Q_{0}$ and define a function $A$ on $\tilde{E}$ by

$$
A(x)=\sup _{\varepsilon>0}\left(\left|T^{\varepsilon}(f d \mu)(x)\right|+\sum_{n \in J}\left|T^{\varepsilon}\left(\varphi_{n}\right)(x)\right|\right),
$$

for $x \in E_{\infty}$, and

$$
A(x)=\sup _{\varepsilon \geq A^{-k(Q)} / 5}\left(\left|T^{\varepsilon}(f d \mu)(x)\right|+\sum_{n \in J}\left|T^{\varepsilon}\left(\varphi_{n}\right)(x)\right|\right),
$$

for $x \in \mathcal{C}(Q), Q \in I_{1} \cup L I$.

Lemma 13.24. We have that

$$
\int_{R\left(Q_{0}\right)} A(x)^{2} d \nu^{+}(x) \leq C \mu\left(Q_{0}\right),
$$

with a constant $C$ that does not depend on $\varepsilon>0$ or $Q_{0}$.

When $x \in E_{\infty},[\mathrm{DM},(* 4.130)$ and $(* 4.131)]$ give that

$$
\begin{aligned}
A(x) \leq & C+C \sum_{n \in J} \sum_{Q \in \mathcal{A}_{n} \cup \mathcal{A}_{n}^{*}} \theta(Q) \mathbf{1}_{E \backslash Q}(x) e_{Q}^{*}(x) \\
& +C \sum_{\substack{n \in J \\
Q \in P L I}} \sum_{\substack{n \in \mathcal{A}_{n}^{*} \\
n}} \theta(Q) \mathbf{1}_{Q}(x) h_{Q}^{*}(x),
\end{aligned}
$$

with the notations of $[\mathrm{DM}]$, that we won't have to make explicit here. Thus

$$
A(x) \leq C+W_{1}^{J}(x)+W_{2}^{J}(x),
$$

where $W_{1}^{J}$ and $W_{2}^{J}$ are as in $(* 5.1)$ and $(* 5.3)$, but where one sums only on the cubes $Q \in \mathcal{R}=I_{1} \cup L I \cup B L I$ that come from indices $n \in J$, i.e., cubes that lie in $\mathcal{A}_{n} \cup \mathcal{A}_{n}^{*}$ for some $n \in J$. By Remarks $* 5.177$ and $* 5.179$, and especially $(* 5.182)$,

$$
\int_{R\left(Q_{0}\right) \cap E_{\infty}} A(x)^{2} d \nu^{+}(x) \leq C \nu^{+}\left(R\left(Q_{0}\right) \cap E_{\infty}\right)+C \mu\left(\bigcup_{n \in J} Q_{n}\right)
$$

$$
\leq C \mu\left(Q_{0}\right)
$$


(See $(* 5.180)$ if you want to check that $\nu^{+}$is the same here as in [DM], and recall that $\nu^{+}=\mu$ on $\left.E_{\infty}\right)$.

Now suppose that $x \in \mathcal{C}(Q)$ for some $Q \in I_{1} \cup L I$. We may use $(* 4.132)$ and $(* 4.133)$ to get that

$$
\begin{aligned}
& A(x) \leq C+C \sum_{n \in J} \sum_{Q \in \mathcal{A}_{n} \cup \mathcal{A}_{n}^{*}} \theta(Q) \tilde{e}_{Q}(x) \\
& +C \sum_{\substack{n \in J \\
Q_{n} \in P L I}} \sum_{Q \in \mathcal{A}_{n}^{*}} \theta(Q) \tilde{h}_{Q}(x) \text {. }
\end{aligned}
$$

(See $(* 4.14)$ and a little below for the definition of $k_{m}$; indeed $k_{m}=$ $k(Q)$ for the cubes $Q \in \mathcal{A}_{m}$.) Then

$$
A(x) \leq C+\tilde{W}_{1}^{J}(x)+\tilde{W}_{2}^{J}(x),
$$

where $\tilde{W}_{1}^{J}$ and $\tilde{W}_{2}^{J}$ are defined like $\tilde{W}_{1}$ and $\tilde{W}_{2}$ in $(* 5.2)$ and $(* 5.4)$, but where we only sum over those cubes $Q \in \mathcal{R}$ that lie in $\mathcal{A}_{n} \cup \mathcal{A}_{n}^{*}$ for some $n \in J$. Now

$$
\begin{aligned}
\int_{R\left(Q_{0}\right) \backslash E_{\infty}} A(x)^{2} d \nu^{+}(x) & =\int_{R\left(Q_{0}\right) \cap\left(\bigcup_{Q \in I_{1} \cup L I} \mathcal{C}(Q)\right)} A(x)^{2} d \nu^{+}(x) \\
& \leq C \nu^{+}\left(R\left(Q_{0}\right)\right)+C \mu\left(\bigcup_{n \in J} Q_{n}\right) \\
& \leq C \mu\left(Q_{0}\right),
\end{aligned}
$$

by $(* 5.182)$ and Lemma 11.13 . Lemma 13.24 follows from this and (13.28).

Now we want to take care of the $T^{\varepsilon}\left(\varphi_{n}\right)$ for which $n \notin J$. We start with the set $J_{1}$ of integers such that $Q_{n}$ does not meet $Q_{0}$.

Denote by $x_{0}$ the "center of $Q_{0}$ ", i.e., the point $x\left(Q_{0}\right)$ of (10.3)(10.5). For each $n \in J_{1}$, set

$$
B_{n}(x)=\left|T^{\varepsilon} \varphi_{n}(x)-T^{\varepsilon} \varphi_{n}\left(x_{0}\right)\right| .
$$

Lemma 13.33. We have that

$$
\sum_{n \in J_{1}} B_{n}(x) \leq C+C Z(x), \quad \text { for } x \in R\left(Q_{0}\right),
$$


where

$$
Z(x)=\int_{E \backslash Q_{0}} \frac{A^{-k\left(Q_{0}\right)}}{|x-y|\left|x_{0}-y\right|} d \mu(y)
$$

To prove the lemma, set

$$
\begin{aligned}
& \text { (13.36) } \quad V(x)=\left\{y \in \mathbb{C}:|x-y|>\varepsilon \text { and }\left|x_{0}-y\right|>\varepsilon\right\}, \\
& (13.37) \quad W(x)=\left\{y \in \mathbb{C} \backslash V(x):|x-y|>\varepsilon \text { or }\left|x_{0}-y\right|>\varepsilon\right\},
\end{aligned}
$$

and then define a function $h$ by

$$
h(y)= \begin{cases}\frac{\left|x-x_{0}\right|}{|x-y|\left|x_{0}-y\right|}, & \text { when } y \in V(x), \\ |x-y|^{-1}+\left|x_{0}-y\right|^{-1}, & \text { when } y \in W(x), \\ 0, & \text { otherwise. }\end{cases}
$$

Obviously

$$
\begin{aligned}
B_{n}(x) & \leq \int h(y)\left|\varphi_{n}(y)\right| \\
& \leq \int_{H_{n}} \rho_{n-1} h d \mu+\theta_{n} \int_{G_{n}} \rho_{n-1} h d \mu+\left|\alpha_{n}\right| \int_{\mathcal{C}_{n}} h d \nu_{n},
\end{aligned}
$$

by (13.20) and because $\|f\|_{\infty} \leq 1$. We want to sum this over $n \in J_{1}$. Notice that the sets $H_{n}$ are disjoint by (10.26) and contained in $E \backslash Q_{0}$ by definition of $J_{1}$. The $\mathcal{C}_{n}$ 's are disjoint too, by (10.29). The sets $G_{n}$ are not necessarily disjoint, but (10.18) says that

$$
\theta_{n} \rho_{n-1}(x)=\rho_{n-1}(x)-\rho_{n}(x), \quad \text { when } x \in G_{n},
$$

so that for a given $x \in E$,

$$
\sum_{n: x \in G_{n}} \theta_{n} \rho_{n-1}(x) \leq 1 .
$$

Thus

$$
\begin{aligned}
\sum_{n \in J_{1}} B_{n}(x) & \leq \int_{\bigcup_{n \in J_{1}} H_{n}} h d \mu+\int_{\bigcup_{n \in J_{1}} G_{n}} h d \mu+C \sum_{n \in J_{1}} \int_{\mathcal{C}_{n}} h d \nu \\
& \leq 2 \int_{E \backslash Q_{0}} h d \mu+C \sum_{n \in J_{1}} \int_{\mathcal{C}_{n}} h d \nu,
\end{aligned}
$$


for all $x \in R\left(Q_{0}\right)$.

Let us first take care of the integrals on $W(x)$. Let $x \in R\left(Q_{0}\right)$ be given. When $\varepsilon>2 \operatorname{diam}\left(R\left(Q_{0}\right) \cup\left\{x_{0}\right\}\right), W(x) \subset B\left(x_{0}, 2 \varepsilon\right)$ and $h(x)=|x-y|^{-1}+\left|x_{0}-y\right|^{-1} \leq 4 \varepsilon^{-1}$ on $W(x)$, and hence

$$
\int_{W(x)} h(y) d \mu(y)+\int_{W(x)} h(y) d \nu(y) \leq C,
$$

be (10.15) (applied to $Q_{0}$ or to a suitable ancestor of $\left.Q_{0}\right)$ and (11.9).

When $\varepsilon \leq 2 \operatorname{diam}\left(R\left(Q_{0}\right) \cup\left\{x_{0}\right\}\right), W(x) \subset B\left(x_{0}, C A^{-k\left(Q_{0}\right)}\right)$, and then

$$
h(y) \leq \frac{C A^{-k\left(Q_{0}\right)}}{|x-y|\left|x_{0}-y\right|}, \quad \text { on } W(x) .
$$

From this and (13.41) we deduce that

$$
\sum_{n \in J_{1}} B_{n}(x) \leq C+C Z(x)+C \sum_{n \in J_{1}} \int_{\mathcal{C}_{n}} \rho(y) d \nu(y),
$$

where

$$
\rho(y)=\frac{A^{-k\left(Q_{0}\right)}}{|x-y|\left|x_{0}-y\right|} .
$$

We still need to control the contribution of the sets $\mathcal{C}_{n}$. Let $n \in J_{1}$ be given, and let $Q \in \mathcal{A}_{n}$. Since $n \in J_{1}, Q_{n}$ does not meet $Q_{0}$, and neither does $Q \subset Q_{n}$. Then

$$
\operatorname{dist}\left(x_{0}, \mathcal{C}(Q)\right) \geq \operatorname{dist}\left(Q_{0}, \mathcal{C}(Q)\right) \geq \operatorname{dist}(\mathcal{C}(Q), E \backslash Q) \geq \frac{99}{100} r(Q),
$$

by (10.28). Hence

$$
\left|x_{0}-z\right| \leq C\left|x_{0}-y\right|, \quad \text { for all } z \in Q \text { and } y \in \mathcal{C}(Q)
$$

Similarly, $\mathcal{C}(Q)$ does not meet $R\left(Q_{0}\right)$, by (11.5) and the fact that the circles $\mathcal{C}(Q), Q \in I_{1} \cup L I$, are disjoint (by (10.29)). Then for all $x \in$ $R(Q)$ we have that

$$
\operatorname{dist}(x, \mathcal{C}(Q)) \geq \operatorname{dist}(R(Q), \mathcal{C}(Q)) \geq \operatorname{dist}(\mathcal{C}(Q), \tilde{E} \backslash \mathcal{C}(Q)) \geq \frac{98}{100} r(Q),
$$


by (11.27), and

$$
|x-z| \leq C|x-y|, \quad \text { for } z \in Q \text { and } y \in \mathcal{C}(Q) .
$$

From (13.46) and (13.47) we deduce that $\rho(y) \leq C \rho(z)$ whenever $y \in$ $\mathcal{C}(Q)$ and $z \in Q$, and then

$$
\begin{aligned}
\sum_{n \in J_{1}} \int_{\mathcal{C}_{n}} \rho(y) d \nu(y) & =\sum_{n \in J_{1}} \sum_{Q \in \mathcal{A}_{n}} \int_{\mathcal{C}(Q)} \rho(y) d \nu(y) \\
& \leq C \sum_{n \in J_{1}} \sum_{Q \in \mathcal{A}_{n}} \int_{Q} \rho(z) d \mu(z) \\
& \leq C \int_{E \backslash Q_{0}} \rho(z) d \mu(y) \\
& =C Z(x),
\end{aligned}
$$

because $\nu(\mathcal{C}(Q)) \leq \mu(Q)$, the cubes $Q$ are disjoint and do not meet $Q_{0}$, and by definition (13.35) of $Z$.

Lemma 13.33 follows from (13.44) and (13.48).

Lemma 13.49. We have

$$
\int_{R\left(Q_{0}\right)} Z(x)^{2} d \nu^{+}(x) \leq C \mu\left(Q_{0}\right) .
$$

We leave the proof of Lemma 13.49 for later, and continue with the proof of (13.18). Lemmas 13.33 and 13.49 will give us enough control on the $T^{\varepsilon}\left(\varphi_{n}\right), n \in J_{1}$ (see later). So we want to switch to the set $J_{2}=\mathbb{N}^{*}-\left(J \cup J_{1}\right)$ of integers $n \geq 1$ such that $Q_{0}$ is strictly contained in $Q_{n}$. Thus $Q_{0} \subset G_{n}$ when $n \in J_{2}$. For each $n \in J_{2}$, set

$$
\begin{aligned}
\psi_{n} & =\varphi_{n}+\theta_{n} \mathbf{1}_{Q_{0}} \rho_{n-1} f d \mu \\
& =-\mathbf{1}_{H_{n}} \rho_{n-1} f d \mu-\theta_{n} \mathbf{1}_{G_{n} \backslash Q_{0}} \rho_{n-1} f d \mu+\alpha_{n} d \nu_{n},
\end{aligned}
$$

(by (13.20)), and then set

$$
B_{n}(x)=\left|T^{\varepsilon} \psi_{n}(x)-T^{\varepsilon} \psi_{n}\left(x_{0}\right)\right|, \quad \text { for } x \in R\left(Q_{0}\right) .
$$

We claim that

$$
\sum_{n \in J_{2}} B_{n}(x) \leq C+C Z(x), \quad \text { for } x \in R\left(Q_{0}\right),
$$


by the same proof as for Lemma 13.33. The main point is still that the sets $H_{n}$ are disjoint and disjoint from $R\left(Q_{0}\right)$, that the integrals against $\alpha_{n} d \nu_{n}$ are controlled by the integrals on $H_{n}$, and that the integrals on the sets $G_{n} \backslash Q_{0}$ sum up by (13.39) and still concern $E \backslash Q_{0}$.

The last piece that we need to study is

$$
\psi=\sum_{n \in J_{2}} \theta_{n} \rho_{n-1} \mathbf{1}_{Q_{0}} f d \mu=(1-\rho) \mathbf{1}_{Q_{0}} f d \mu,
$$

where $\rho$ denotes the constant value of $\rho_{n_{0}}$ on $Q_{0}$, where $n_{0}$ is the largest integer in $J_{2}$. (If $J_{2}$ is empty, we don't need to worry but we can also take $\rho=1$ and $\psi=0$.) The last equality in (13.54) comes from (13.39). For each $x \in R\left(Q_{0}\right)$, set

$$
D(x)=E \cap B\left(x, \operatorname{diam}\left(Q_{0} \cup R\left(Q_{0}\right)\right)+A^{-k\left(Q_{0}\right)}\right) .
$$

By $(* 4.97)$ or $(* 4.98)$,

$$
\left|T^{\varepsilon}\left(\mathbf{1}_{E \backslash D(x)} f d \mu\right)(x)\right| \leq C,
$$

because it is a $T^{\tilde{\varepsilon}}(f d \mu)(x)$ for some $\tilde{\varepsilon} \geq A^{-k\left(Q_{0}\right)}$; next

$$
\left|T^{\varepsilon}\left(\mathbf{1}_{E \backslash D\left(x_{0}\right)} f d \mu\right)(x)\right| \leq C,
$$

by (13.56), and because the difference between the left-hand sides of (13.56) and (13.57) is controlled by

$$
\int_{\Delta} \frac{d \mu(y)}{|x-y|} \leq C,
$$

where $\Delta=\left(D\left(x_{0}\right) \backslash D(x)\right) \cup\left(D(x) \backslash D\left(x_{0}\right)\right)$. This last estimate uses (10.15). Now assume that $x \in R\left(Q_{0}\right) \cap E_{\infty}$ or $x \in R\left(Q_{0}\right) \backslash E_{\infty}$ and $x \in \mathcal{C}(Q)$ for some $Q \in I_{1} \cup L I$ such that $\varepsilon \geq A^{-k(Q)} / 5$. Then $\left|T^{\varepsilon}(f d \mu)(x)\right| \leq C$ by $(* 4.97)$ or $(* 4.98)$, and hence

$$
\begin{aligned}
\left|T^{\varepsilon} \psi(x)\right| & \leq\left|T^{\varepsilon}(f d \mu)(x)\right|+\left|T^{\varepsilon}\left(\mathbf{1}_{E \backslash Q_{0}} f d \mu\right)(x)\right| \\
& \leq C+\left|T^{\varepsilon}\left(\mathbf{1}_{E \backslash D\left(x_{0}\right)} f d \mu\right)(x)\right|+\int_{D\left(x_{0}\right) \backslash Q_{0}} \frac{d \mu(y)}{|x-y|} \\
& \leq C+\int_{D\left(x_{0}\right) \backslash Q_{0}} \frac{d \mu(y)}{|x-y|} .
\end{aligned}
$$


The following lemma will be useful; we shall prove it later, at the same time as Lemma 13.49 .

Lemma 13.59. Set

$$
Z_{1}(x)=\int_{D\left(x_{0}\right) \backslash Q_{0}} \frac{d \mu(y)}{|x-y|}, \quad \text { for all } x \in R\left(Q_{0}\right) \text {. }
$$

Then

$$
\int_{R\left(Q_{0}\right)} Z_{1}(x)^{2} d \nu^{+}(x) \leq C \mu\left(Q_{0}\right) .
$$

We are now ready to prove (13.18) (modulo the two lemmas). Take

$$
m_{0}=\sum_{n \in J_{1}} T^{\varepsilon} \varphi_{n}\left(x_{0}\right)+\sum_{n \in J_{2}} T^{\varepsilon} \psi_{n}\left(x_{0}\right) .
$$

For each $x \in R\left(Q_{0}\right) \cap E_{\infty}=Q_{0} \cap E_{\infty}$ and $\varepsilon>0$,

$$
\begin{aligned}
\left|T_{\varepsilon} g(x)-m_{0}\right|= & \left|T^{\varepsilon}(g d \nu)(x)-m_{0}\right| \\
\leq & A(x)+\sum_{n \in J_{1}}\left|T^{\varepsilon} \varphi_{n}(x)-T^{\varepsilon} \varphi_{n}\left(x_{0}\right)\right| \\
& +\sum_{n \in J_{2}}\left|T^{\varepsilon} \psi_{n}(x)-T^{\varepsilon} \psi_{n}\left(x_{0}\right)\right|+\left|T^{\varepsilon} \psi(x)\right| \\
\leq & A(x)+\sum_{n \in J_{1} \cup J_{2}} B_{n}(x)+C+Z_{1}(x) \\
\leq & A(x)+C+C Z(x)+Z_{1}(x),
\end{aligned}
$$

by (13.19), (13.22), (13.51) and (13.54) (to get that $\sum_{n \in J_{2}} \varphi_{n}=$ $\left.\sum_{n \in J_{2}} \psi_{n}+\psi\right),(13.32)$ and (13.52), (13.58), Lemma 13.33, and (13.53).

When $x \in R\left(Q_{0}\right) \backslash E_{\infty}$ and $x \in \mathcal{C}(Q)$ for some $Q \in I_{1} \cup L I$, and we suppose in addition that $\varepsilon \geq A^{-k(Q)} / 5$, we can use (13.23) instead of (13.22), and the same computations as for (13.62) yield

$$
\left|T_{\varepsilon} g(x)-m_{0}\right| \leq A(x)+C+C Z(x)+Z_{1}(x) .
$$

When $x \in \mathcal{C}(Q)$ and $\varepsilon<A^{-k(Q)} / 5$, set $\varepsilon^{\prime}=A^{-k(Q)} / 5$ and observe that

$$
\begin{aligned}
\left|T_{\varepsilon} g(x)-T_{\varepsilon^{\prime}} g(x)\right| & =\left|\int_{\left\{\varepsilon<|x-y|<\varepsilon^{\prime}\right\}} \frac{g(y) d \nu(y)}{x-y}\right| \\
& =\left|\int_{\left\{y \in \mathcal{C}(Q), \varepsilon<|x-y|<\varepsilon^{\prime}\right\}} \frac{\alpha_{m} d \nu_{m}(y)}{x-y}\right| \leq C,
\end{aligned}
$$


by $(11.27),(10.31),(10.25),(10.20)$, and elementary properties of truncated Cauchy integrals on circles, and where $m$ denotes the integer such that $Q \in \mathcal{A}_{m}$. Thus (13.63) holds also when $\varepsilon<A^{-k(Q)} / 5$, even though with a slightly larger constant $C$. Altogether, (16.63) holds for all $x \in R\left(Q_{0}\right)$ (and all $\varepsilon>0$ ).

Now (13.18) follows from Lemmas 13.24, 13.49, 13.59, plus the fact that $\nu^{+}\left(R\left(Q_{0}\right)\right) \leq C \mu\left(Q_{0}\right)$, by Lemma 11.13. Because of Lemma 13.17, our proof of (13.6) will be complete as soon as we establish the two lemmas.

First consider the function $Z(x)$ of Lemma 13.33. We claim that

$$
Z(x) \leq C+Z_{1}(x), \quad \text { for all } x \in R\left(Q_{0}\right),
$$

where $Z_{1}$ is as in Lemma 13.59. Let $D\left(x_{0}\right)$ be as in (13.55) and the definition of $Z_{1}$. Then

$$
\int_{E \backslash D\left(x_{0}\right)} \frac{A^{-k\left(Q_{0}\right)}}{|x-y|\left|x_{0}-y\right|} d \mu(y) \leq C,
$$

by the same computation as for (8.1), because $\left(|x-y|\left|x_{0}-y\right|\right)^{-1} \leq$ $C\left|x_{0}-y\right|^{-2}$ on the domain of integration and by (10.15), applied to $Q_{0}$ and its ancestors.

So we may concentrate on

$$
Z_{2}(x)=\int_{D\left(x_{0}\right) \backslash Q_{0}} \frac{A^{-k\left(Q_{0}\right)}}{|x-y|\left|x_{0}-y\right|} d \mu(y) .
$$

But $\left|x_{0}-y\right| \geq A^{-k\left(Q_{0}\right)} / 2$ on $D\left(x_{0}\right) \backslash Q_{0}$, by (10.3) and (10.4), and so $Z_{2}(x) \leq 2 Z_{1}(x)$. This proves our claim (13.65).

Obviously Lemma 13.49 will follow from Lemma 13.59 and (13.65), because $\nu^{+}\left(R\left(Q_{0}\right)\right) \leq \mu\left(Q_{0}\right)$ by Lemma 11.13 .

We now prove Lemma 13.59. The argument is quite similar to estimates for functions $h_{Q}^{*}$ that were done at the beginning of [DM, Section 5.1], but we give the argument here because some of the computations in $[\mathrm{DM}]$ are much more general than what we need here.

First we want to reduce to an integral on $Q_{0}$ (rather than $R\left(Q_{0}\right)$ ). For each $x \in Q_{0}$, set

(13.67) $r(x)=\inf \left\{A^{-k}:\right.$ there is a cube $Q \in \Delta_{k}^{0}$ that contains $\left.x\right\}$.

The main point of this definition is that

$$
\mu(B(x, r)) \leq C r, \quad \text { for all } r \geq r(x),
$$


by (10.15). Also note that

$$
r(x)=0, \quad \text { on } E_{\infty} \cap Q_{0},
$$

because $E_{\infty}$ does not meet any cube of $I_{1} \cup L I$. Next set

$$
h(x)=\mathbf{1}_{Q_{0}}(x) \int_{D\left(x_{0}\right) \backslash Q_{0}} \frac{d \mu(y)}{r(x)+|x-y|} .
$$

We want to check that

$$
\int_{R\left(Q_{0}\right)} Z_{1}(x)^{2} d \nu^{+}(x) \leq C \int_{Q_{0}} h(x)^{2} d \mu(x) .
$$

For $x \in E_{\infty} \cap R\left(Q_{0}\right), r(x)=0$ and $Z_{1}(x)=h(x)$; for the corresponding part of the integral, there is nothing to check because $\nu^{+} \leq \mu$ on $E_{\infty}$.

Now let $Q \in I_{1} \cup L I$ be given, with $Q \subset Q_{0}$, and let us look at the contribution of $\mathcal{C}(Q)$. For each $x \in \mathcal{C}(Q)$,

$$
\operatorname{dist}\left(x, D\left(x_{0}\right) \backslash Q_{0}\right) \geq \operatorname{dist}(\mathcal{C}(Q), E \backslash Q) \geq \frac{99}{100} r(Q),
$$

by (10.28), and hence

$(13.72) r(z)+|z-y| \leq A^{-k(Q)}+|z-y| \leq 100 r(Q)+|x-y| \leq C|x-y|$,

for all $y \in D\left(x_{0}\right) \backslash Q_{0}$ and all $z \in Q$. Then $Z_{1}(x) \leq C h(z)$ for all $z \in Q$, and

$$
\int_{\mathcal{C}(Q)} Z_{1}(x)^{2} d \nu^{+}(x) \leq C \int_{Q} h^{2}(z) d \mu(z),
$$

because $\nu^{+}(\mathcal{C}(Q))=\mu(Q)$. When we sum this over the (disjoint) cubes $Q \in I_{1} \cup L I$ that are contained in $Q_{0}$, we obtain that

$$
\int_{R\left(Q_{0}\right) \backslash E_{\infty}} Z_{1}(x)^{2} d \nu^{+}(x) \leq C \int_{Q_{0}} h(z)^{2} d \mu(z)
$$

(by (11.5)); our claim (13.71) follows from this and the trivial estimate for $E_{\infty}$ mentionned above.

Because of (13.71), Lemma 13.59 will follow as soon as we show that

$$
\int_{Q_{0}} h(x)^{2} d \mu(x) \leq C \mu\left(Q_{0}\right)
$$


To prove this we decompose $Q_{0}$ into its maximal good subcubes $R$, $R \in S\left(Q_{0}\right)$. The decomposition is the same as in Section 8, even though $\mu$ is a slightly different measure now (that does not satisfy (3.1)). In particular, the analogue of (8.10) in this context holds, with the same proof. (See Lemma $* 5.28$.) For each $R \in S\left(Q_{0}\right)$, set

$$
h_{R}(x)=\mathbf{1}_{R}(x) \int_{2 R \backslash R} \frac{d \mu(y)}{r(x)+|x-y|},
$$

where $2 R$ is as in $(7.7)-(7.8)$ or in $(* 4.79)$. This is almost the same function as in $[\mathrm{DM}]($ see $(* 5.8))$, with the only minor difference that we may have chosen $r(x)$ a little larger than the one in [DM]. (See in particular $(* 5.5)$ and $(* 5.7)$.) This difference does not disturb us, because our function $h_{R}$ is slightly smaller than the one in [DM], and the estimates from $[\mathrm{DM}]$ will work even better for it. Now we claim that

$$
h(x) \leq C+h_{Q_{0}}(x) \leq C^{\prime}+h_{R}(x),
$$

when $x \in R, R \in S\left(Q_{0}\right)$. The first inequality is an easy consequence of the fact that $|x-y| \geq A^{-k\left(Q_{0}\right)}$ on $D\left(x_{0}\right) \backslash 2 Q_{0}$, so that

$$
h(x)-h_{Q_{0}}(x)=\int_{D\left(x_{0}\right) \backslash 2 Q_{0}}|x-y|^{-1} d \mu(y) \leq A^{k\left(Q_{0}\right)} \mu\left(D\left(x_{0}\right)\right) \leq C,
$$

by (10.15). The second inequality comes directly from Lemma $* 5.36$. The fairly easy proof is quite similar to arguments used earlier in this paper: because all the cubes $Q$ such that $R \subset Q \subset Q_{0}$ and $Q \neq R$ are bad, the contribution to $h_{Q_{0}}(x)$ of the annular shells at distance $\sim A^{-k\left(Q_{0}\right)-\ell}, \ell \leq k(R)-k\left(Q_{0}\right)$, from $x$ decrease rapidly; the main contribution comes from $\ell=0$ and is less than $C$ by (10.15). (See [DM] for details.)

Next, for each $R \in S\left(Q_{0}\right)$ and each $x \in R$,

$$
h_{R}(x) \leq C\left(1+\log \left(1+A^{-k(R)} \operatorname{dist}(x, 2 R \backslash R)^{-1}\right)\right) .
$$

This is $(* 5.24)$, and it follows from a rather brutal computation using dyadic annular shells and the density estimate (13.68). The logarithm is an estimate of the number of shells that we need to cover the domain of integration. Finally,

$$
\int_{R} h_{R}(x)^{2} d \mu(x) \leq C \mu(90 B(R)) \leq C \mu(R) .
$$


This follows fairly easily from (13.78) and (10.8), plus the fact that $R$ is a good cube. This is also a consequence of Lemma $* 5.22$. Now

$$
\begin{aligned}
\int_{Q_{0}} h(x)^{2} d \mu(x) & =\sum_{R \in S\left(Q_{0}\right)} \int_{R} h(x)^{2} d \mu(x) \\
& \leq 2 \sum_{R} \int_{R}\left(h_{R}(x)^{2}+C\right) d \mu(x) \\
& \leq C \sum_{R} \mu(R)=C \mu\left(Q_{0}\right),
\end{aligned}
$$

by (8.10) (or Lemma $* 5.28)$, (13.77), and (13.79).

This completes our proof of (13.75); Lemma 13.59, Lemma 13.49, and our main estimate (13.6) follow.

At this point we may return to the description given in Section 1: the estimate (1.11) follows readily from (13.6), and we may conclude as in the introduction.

This complete our proof of Theorem 1.1.

Acknowledgements. The author wishes to thank Pertti Mattila for initiating this project, pleasant and valuable discussions, and for carefully reading the manuscript. He is also grateful to F. Nazarov, S. Treil, and A. Volberg for communicating some of their ideas, which allowed a nontrivial simplification of Section 9, and to Josette Dumas, who kindly typed the paper.

\section{References.}

[Ah] Ahlfors, L., Bounded analytic functions. Duke Math. J. 14 (1947), 1-11.

[AT] Auscher, P., Tchamitchian, P., Bases d'ondelettes sur les courbes cordearc, noyau de Cauchy et espaces de Hardy associés. Revista Mat. Iberoamericana 5 (1989), 139-170.

[Ca] Calderón, A. P., Cauchy integrals on Lipschitz curves and related operators. Proc. Nat. Acad. Sci. USA 74 (1977), 1324-1327.

[CJS] Coifman, R. R., Jones, P. and Semmes, S., Two elementary proofs of the $L^{2}$-boundedness of Cauchy integrals on Lipschitz curves. J. Amer. Math. Soc. 2 (1989), 553-564. 
[Ch1] Christ, M., Lectures on singular integral operators. Regional conference series in mathematics 77, AMS 1990.

[Ch2] Christ, M., A $T(b)$ theorem with remarks on analytic capacity and the Cauchy integral. Colloq. Math. 60/61 (1990), 1367-1381.

[CM] Coifman, R., Meyer, Y., Wavelets, Calderón-Zygmund and multilinear operators. Cambridge Studies in Advanced Mathematics 48. Cambridge University Press, 1997.

[Da] David, G., Wavelets and singular integrals on curves and surfaces. Lecture notes in Math. 1465, Springer-Verlag 1991.

[DJS] David, G., Journé, J.-L. and Semmes, S., Opérateurs de CalderónZygmund, fonctions para-accrétives et interpolation. Revista Mat. Iberoamericana 1 (1985), 1-56.

[DM] David, G. and Mattila, P., Removable sets for Lipschitz harmonic functions in the plane. Preprint.

[Ga] Garnett, J., Analytic capacity and measure. Lecture Notes in Math. 297, Springer-Verlag 1972.

[HH] In: Linear and Complex Analysis Problem Book. Edited by Havin, V. P., Hruščhëv, S. V., Nikolskii, N. K. Lecture Notes in Math. 1043, Springer-Verlag, 1984.

[JM] Jones, P. W., Murai, T., Positive analytic capacity but zero Buffon needle probability. Pacific J. Math. 133 (1988), 99-114.

[Le] Leger, J.-C., Menger curvature and rectifiability. Preprint, Univ. ParisSud, Orsay.

[Li] Lin, Y., Menger curvature, singular integrals and analytic capacity. Ann. Acad. Sci. Fenn. Ser. AI Dissertationes 111 (1997).

[Ma1] Mattila, P., Smooth maps, null-sets for integralgeometric measure and analytic capacity. Ann. of Math. 123 (1986), 303-309.

[Ma2] Mattila, P., Geometry of sets and measures in Euclidean spaces. Cambridge studies in advanced mathematics 44. Cambridge Univ. Press, 1995.

[MMV] Mattila, P., Melnikov, M. S., Verdera, J., The Cauchy integral, analytic capacity, and uniform rectifiability. Ann. of Math. 144 (1996), 127-136.

[MP] Mattila, P. and Paramonov, P. V., On geometric properties of harmonic Lip 1 -capacity. Pacific J. Math. 171 (1995), 469-491.

[Me] Melnikov, M., Analytic capacity: discrete approach and curvature of measure. Sbornik Math. 186 (1995), 827-846.

[MV] Melnikov, M. and Verdera, J., A geometric proof of the $L^{2}$ boundedness of the Cauchy integral on Lipschitz graphs. Internat. Math. Research Notices 7 (1995), 325-331. 
[My] Meyer, Y., Ondelettes et opérateurs II: Opérateurs de Calderón-Zygmund. Actualités mathématiques. Hermann, 1990.

[NVT] Nazarov, F., Treil, S., Volberg, A. Cauchy integral and Calderón-Zygmund operators on non homogeneous spaces. Preprint, Michigan State Univ. 1997.

[O'] O'Neil, T., A local version of the projection theorem. Proc. London Math. Soc. 73 (1996), 68-104.

[Pa] Pajot, H., Conditions quantitatives de rectifiabilité. Bull. Soc. Math. France 125 (1997), 1-39.

[St] Stein, E. M., Singular integrals and differentiability properties of functions. Princeton Univ. Press, 1970.

[To] Tolsa, X., $L^{2}$-boundedness of the Cauchy integral operator for continuous measures. Preprint, Univ. Autonoma de Barcelona, 1997.

[Uy1] Uy, N. X., Removable sets of analytic functions satisfying a Lipschitz condition. Arkiv. Math. 17 (1979), 19-27.

[Uy2] Uy, N. X., An extremal problem on singular integrals. Amer. J. Math. 102 (1980), 279-290.

[Uy3] Uy, N. X., A removable set for Lipschitz harmonic functions. Michigan Math. J. 37 (1990), 45-51.

[Ve] Verdera, J., Removability, capacity and approximation. In Complex potential theory. NATO ASI Series, Kluwer Acad. Publ., 1994, 419-473.

[Vi] Vitushkin, A. G., The analytic capacity of sets in problems of approximation theory. Uspekhi Mat. Nauk 22 (1967), 141-199. English translation in Russian Math. Surveys $\mathbf{2 2}$

Recibido: 21 de agosto de 1.997

Guy David

Université de Paris-Sud

Mathématiques - Bât. 425

91405 Orsay Cedex, FRANCE

et

Institut Universitaire de France

guy.david@math.u-psud.fr 